\title{
APPLICATIONS OF RADIOTRACER IN PLANT BIOLOGY
}

\author{
A Dissertation \\ Presented to \\ The Faculty of the Graduate School \\ University of Missouri-Columbia \\ In Partial Fulfillment \\ of the Requirements for the Degree \\ Doctor of Philosophy \\ by \\ LIHUI SONG \\ Dr. Silvia Jurisson and Dr. Gary Stacey, Dissertation Supervisors \\ December 2013
}


The undersigned, appointed by the dean of the Graduate School, have examined the dissertation entitled

\section{APPLICATIONS OF RADIOTRACER IN PLANT BIOLOGY}

presented by Lihui Song,

a candidate for the degree of [doctor of philosophy of Chemistry],

and hereby certify that, in their opinion, it is worthy of acceptance.

Professor Silvia Jurisson

Professor Gary Stacey

Professor David Robertson

Professor Timothy Glass

Professor Richard Ferreri 


\section{To My loves,}

I would like to dedicate this work to my dear family who have been loving me and supporting me unconditionally, even though I have not spent any New Year eves with them during the last five years. I also would like to dedicate this work to my loved

fiancé, Noah Marchal, for his understanding, support, patience and help with revision during the dissertation writing process. 


\section{ACKNOWLEDGEMENTS}

There are so many people who helped me create new neuron connections in my brain throughout my graduate career and all of them deserve more than just the ink on one page of my dissertation.

First, I would like to thank my advisor, Dr. Silvia Jurisson, and my co-advisors, Dr. Gary Stacey and Dr. Richard Ferreri, for their guidance, patience, encouragement and revisions on my dissertation. Gary led me to the door of this research opportunity, Silvia welcomed me in, and Rich taught me hand by hand how to conduct radiotracer studies on plants. Without them, I would not have gained the knowledge and experience I had today and figured out what I love to do for my future career. I also would like to thank my dissertation committee members, Dr. Timothy Glass and Dr. David Robertson for their continuous support and their help with the revision of my dissertation. Another person I would like to thank here is Minviluz (Bing) G. Stacey. Although she is not in my dissertation committee, she taught me how to conduct plant biological studies and other important molecular biology techniques, especially helped me with the study mentioned in Chapter 2.

In addition, I would like to thank Dr. Jurisson's Group, Dr. Stacey’s Group and Dr. Ferreri's Group. Some of them helped me a lot with experimental design and actual experiments, and some of them are my spiritual support. I have enjoyed every one of their companies during my graduate career.

There are also facilities I would like to acknowledge for conducting analyses for my work. They are MU Research Reactor (David J. Robertson, James Guthrie and Gang Li) and Environmental Molecular Sciences Laboratory/ Pacific Northwest National 
Laboratory (EMSL/PNNL; Lizabeth Alexander) for the ICP-MS analyses; MU Molecular Cytology Core (Yan Liang) for providing the microscopic imaging services.

At last, I would like to thank MU Chemistry Department and Department of Energy Biological and Environmental Research (DOE-BER) program for the financial supports. 


\section{Table of Contents}

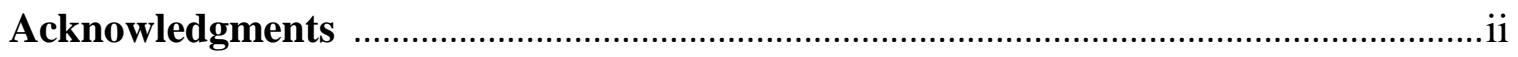

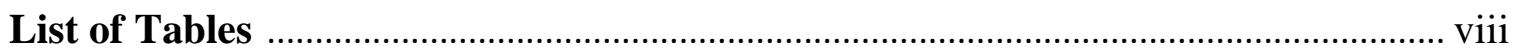

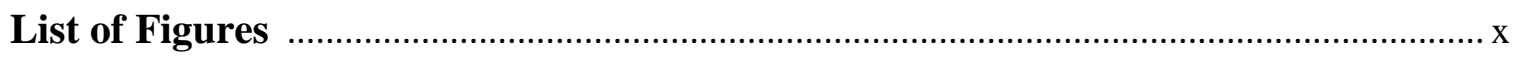

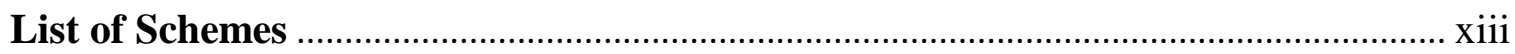

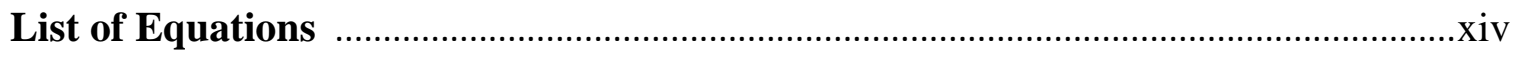

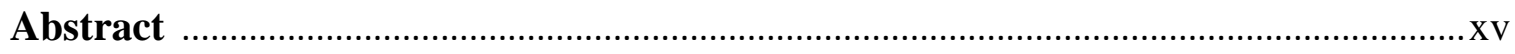

\section{Chapter 1: Introduction}

1.1 General Concepts of Radiotracer ........................................................................... 1

1.2 Techniques used for Radiation Detection ........................................................... 3

1.3 Advantages and Disadvantages of Radiotracer Studies .......................................... 6

1.4 Underlying Assumptions in Design of Radiotracer Experiments ........................... 6

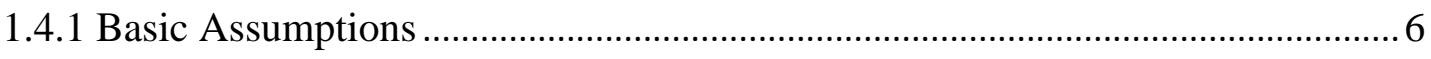

1.4.2 Evaluation of the Feasibility of Radiotracer Experiments .................................. 8

1.5 History of Plant Biological Studies using Radioactive Isotopes ............................... 9

1.6 Radiotracer Studies in This Dissertation ........................................................... 10

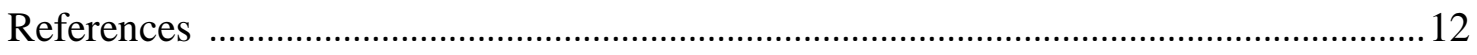

\section{Chapter 2: Accumulation of Heavy Metals by the Arabidopsis opt3-2 Mutant}

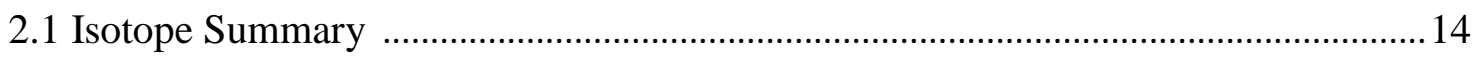

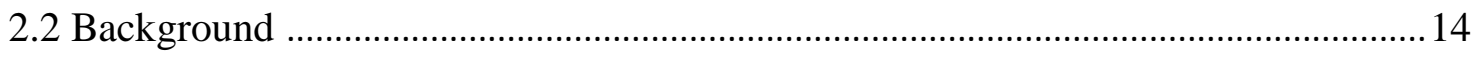

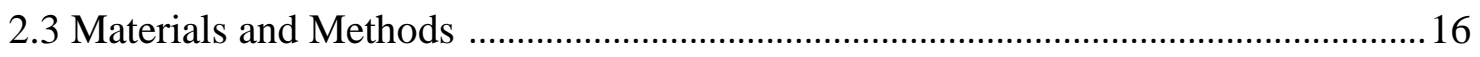

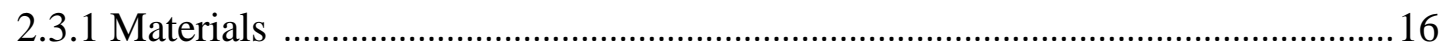

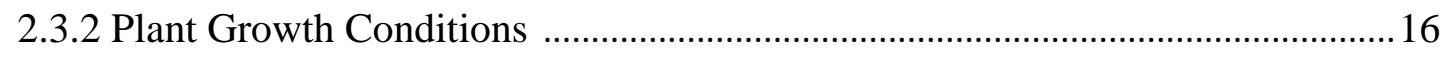




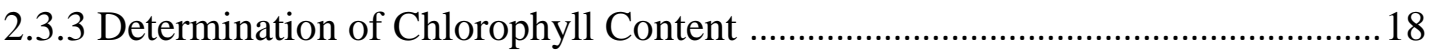

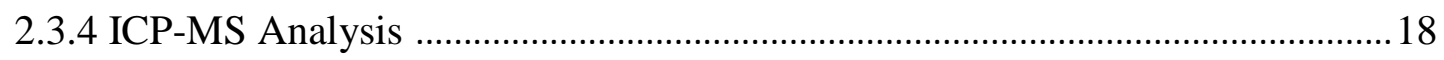

2.3.5 Radioactive ${ }^{203} \mathrm{~Pb}^{2+}$ Uptake Assay and Imaging ................................................ 19

2.3.6 Yeast Transformation and Growth Assays ................................................... 19

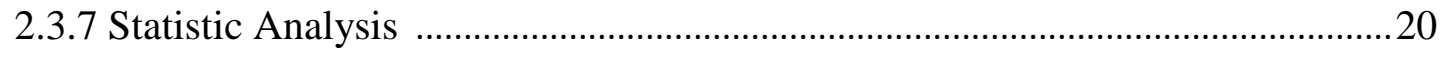

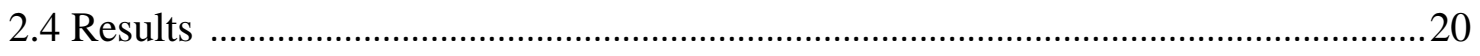

2.4.1 Toxic Effects of Heavy Metals on Plant Growth ...............................................20

2.4.2 Heavy Metal Quantification in Plant Tissues by ICP-MS ................................21

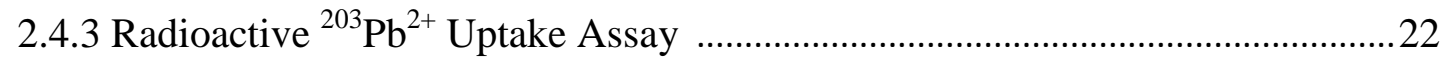

2.4.4 IRT1 does not Mediate Pb or Hg Uptake .........................................................22

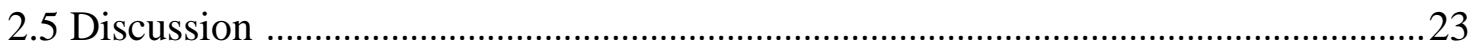

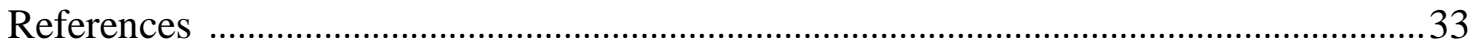

\section{Chapter 3: Dynamics of Sugar Metabolism and Re-location in Arabidopsis Starch Mutants Using ${ }^{11} \mathrm{CO}_{2}$}

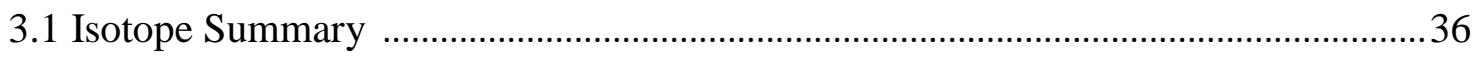

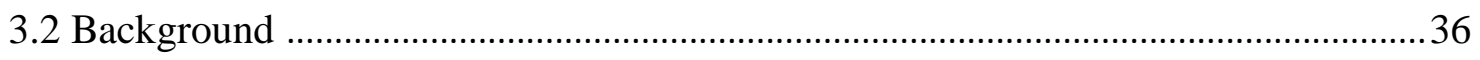

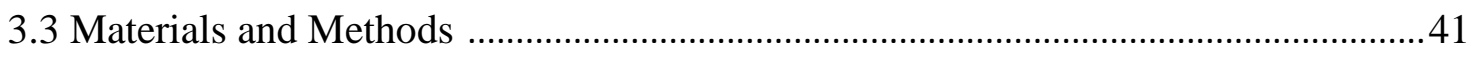

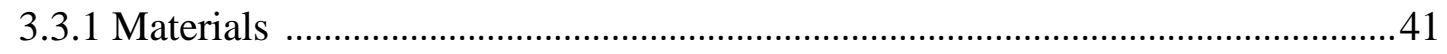

3.3.2 Plant Growth Conditions ............................................................................. 41

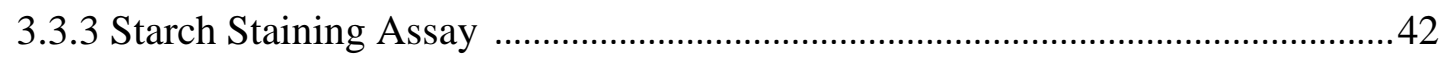

3.3.4 ${ }^{11} \mathrm{CO}_{2}$ Production, Pulsing and Incubation Apparati ............................................42

3.3.5 Tissue Extraction and Soluble Sugar Analysis ................................................43

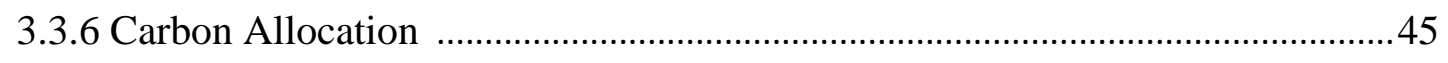

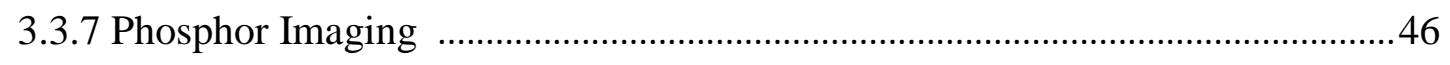

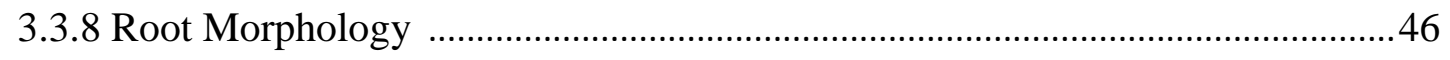




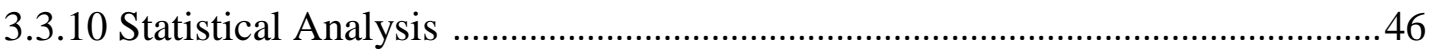

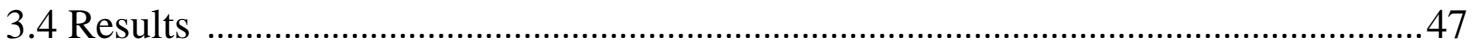

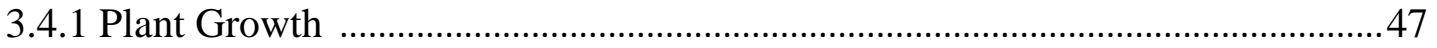

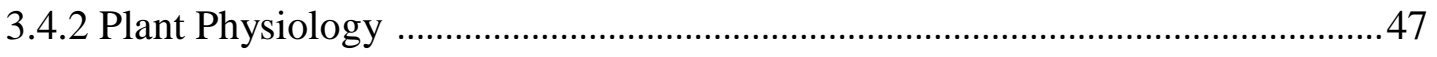

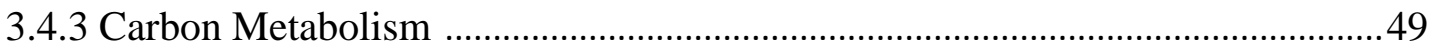

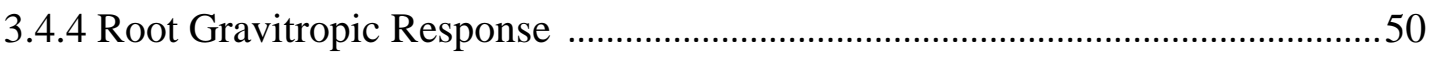

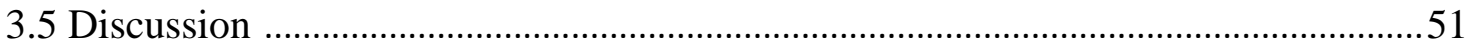

3.5.1 Carbon Metabolism and Allocation ................................................................ 51

3.5.2 Root Morphology and Gravitropic Response .................................................54

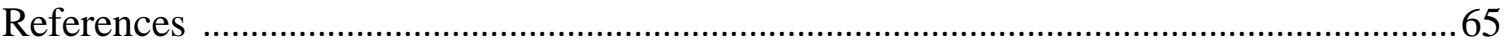

\section{Chapter 4: Effect of Fe Statuse on Carbon Metabolism in Arabidopsis}

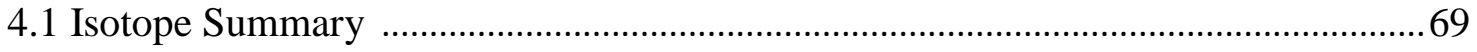

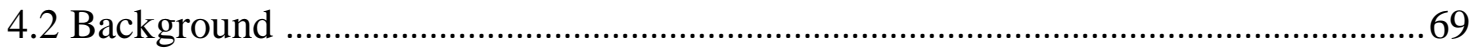

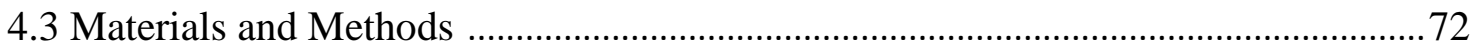

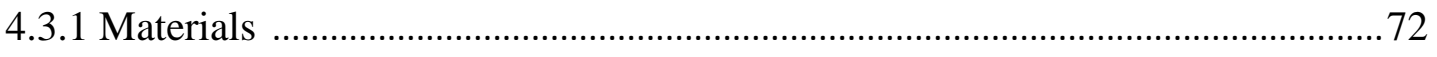

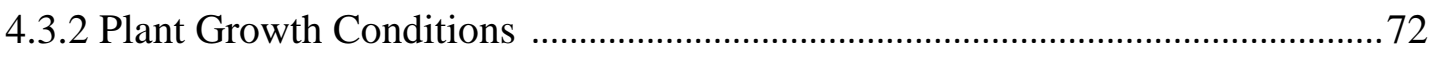

4.3.3 Chlorophyll Content Determination ........................................................... 73

4.3.4 Proton Concentration Measurements in the Rhizosphere ..................................74

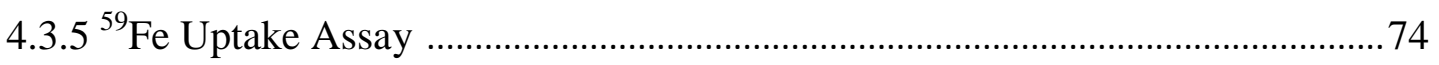

4.3.6 ${ }^{11} \mathrm{CO}_{2}$ Production, Pulsing and Incubation Apparatus .......................................... 75

4.3.7 Tissue Extraction and Soluble Sugar Analysis ...................................................

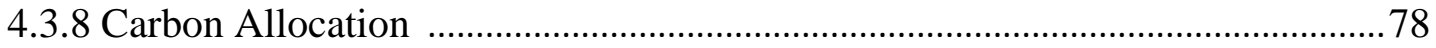

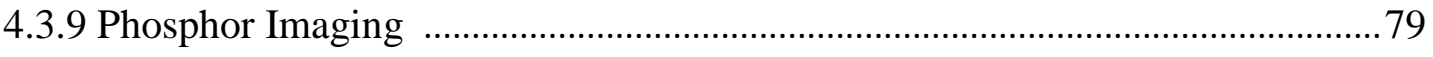

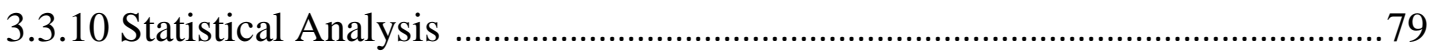


4.4 Results

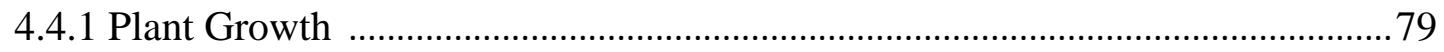

4.4.2 Acidification of Rhizosphere through Root Exudation ...................................... 80

4.4.3 ${ }^{59} \mathrm{Fe}$ Uptake under Various Levels of External Fe Availability ...........................81

4.4.4 Carbon Fixation, Metabolism and Allocation .................................................... 81

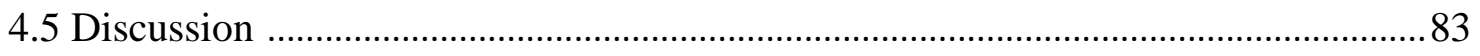

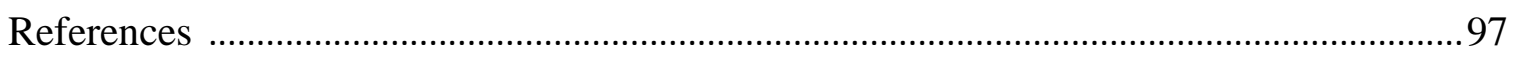

Chapter 5: Conclusions and Future Studies ........................................................... 101

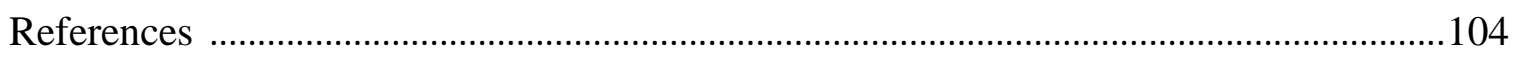

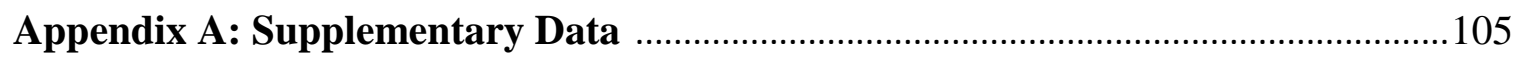

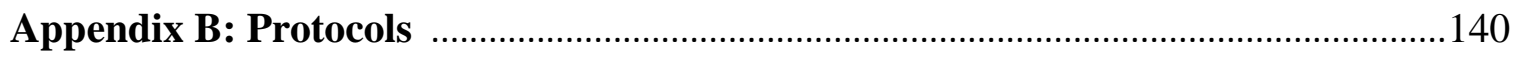

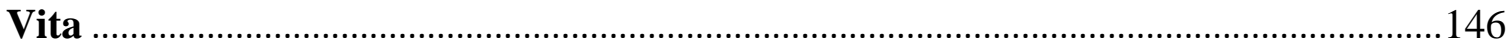




\section{List of Tables}

Page

Table 2-1. Fractions of total ${ }^{203} \mathrm{~Pb}$ taken up in the plant transported into roots and shoots of the WT (col-0) and the opt3-2 mutant

Table A1-1. Heavy metal treatments tested on the opt3-2 mutant plants ....................... 105

Table A1-2. Experimental parameters for ${ }^{35}$ S-glutathione uptake assay ........................118

Table A1-3. Data of root / shoot growth of plants under $\mathrm{Hg}$ or $\mathrm{Pb}$ treatments ............... 119

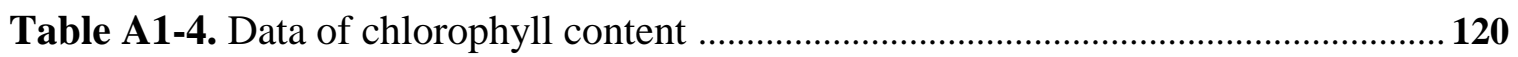

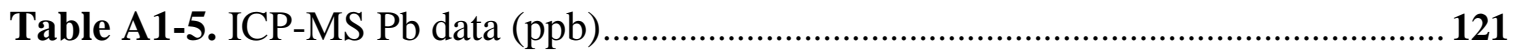

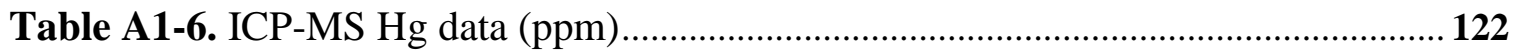

Table A1-7. ${ }^{203} \mathrm{~Pb}$ distribution in opt $3-2$ mutant and WT plants ......................................123

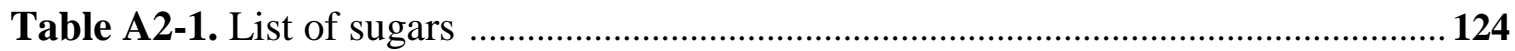

Table A2-2. Data of Chlorophyll content …............................................................... 125

Table A2-3. Comparison of root elongation among three starch mutants and wild type Arabidopsis seedlings

Table A2-4. Comparison of root gravitropic responses among three starch mutants and wild type Arabidopsis seedlings

Table A2-5. Percentage of ${ }^{11} \mathrm{C}$ sugar partitioning in the starch mutants and the wild type

Table A2-6. ${ }^{12} \mathrm{C}$ sugar partitioning in the starch mutants and the wild type

Table A2-7. Percentage of total fixed ${ }^{11} \mathrm{C}$ assimilated into insoluble carbohydrates ..... 130

Table A2-8. ${ }^{11} \mathrm{C}$-carbohydrate relocation to belowground and root exudates 130

Table A3-1. Chlorophyll content 131

Table A3-2. ${ }^{59} \mathrm{Fe}$ uptake under various levels of external Fe availability 132

Table A3-3. Total ${ }^{11} \mathrm{CO}_{2}$ fixation 133 
Table A3-4. ${ }^{11} \mathrm{C}$-sugar partitioning in the leaves of three types of Arabidopsis seedlings

Table A3-5. ${ }^{11} \mathrm{C}$-carbohydrate relocation to belowground and root exudates 138 


\section{List of Figures}

Page

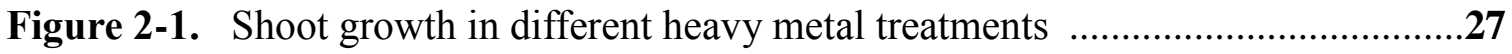

Figure 2-2. Root growth in different heavy metal treatments ..................................28

Figure 2-3. ICP-MS shows the amount of heavy metals in different above-ground tissues of both col-0 and opt3-2 mutant

Figure 2-4. Comparison of different yeast transformants grown under various concentrations of heavy metal treatments

Figure 2-5. Phosphor image of ${ }^{203} \mathrm{~Pb}$ distribution in opt3-2 mutant and wild type plants

Figure 2-6. ${ }^{203} \mathrm{~Pb}$ distribution in opt3-2 mutant and wild type plants

Figure 3-1. Brief summary of carbon fixation and downstream carbon metabolic pathway

Figure 3-2. The apparati used for ${ }^{11} \mathrm{CO}_{2}$ pulsing and incubation

Figure 3-3. Comparison of root/shoot growth and chlorophyll content among the three starch mutants and WT

Figure 3-4. Comparison of root elongation and gravitropic responses among three starch mutants and wild type Arabidopsis seedlings

Figure 3-5. Carbon-11 phosphor imaging of the three starch mutants and the wild type

Figure 3-6. Iodine staining assays of starch in three starch mutants and wild type Arabidopsis

Figure 3-7. Iodine staining assay of starch in the root tips of three starch mutants and wild type Arabidopsis

Figure 3-8. ${ }^{11} \mathrm{C}$-carbohydrate relocation to belowground and root exudation

Figure 3-9. ${ }^{12} \mathrm{C}$ and ${ }^{11} \mathrm{C}$ sugar partitioning in the starch mutants and the wild type ......63

Figure 3-10. Percentage of total fixed ${ }^{11} \mathrm{C}$ assimilated into insoluble carbohydrates ......64 
Figure 4-1. Two strategies for iron uptake in plants

Figure 4-2. Experimental set-up for ${ }^{59} \mathrm{Fe}$ uptake

Figure 4-3. Comparison of root/shoot growth and chlorophyll content among the mutants and WT

Figure 4-4. Measurement of root exudate $\mathrm{pH}$ of Arabidopsis seedlings (wt, opt3-2 and irt1-1) grown under various concentrations of Fe treatments using a $\mathrm{pH}$ indicator

Figure 4-5. ${ }^{59} \mathrm{Fe}$ uptake under various levels of external Fe availability

Figure 4-6. Total ${ }^{11} \mathrm{CO}_{2}$ fixation

Figure 4-7. ${ }^{11} \mathrm{C}$-sugar partitioning in the leaves of three types of Arabidopsis seedlings

Figure 4-8. Allocation of ${ }^{11} \mathrm{C}$-photosynthates to the belowground 95

Figure 4-9. Root exudation .96

Figure 5-1. A conceptual model used to interpretate ${ }^{12} \mathrm{C}$ and ${ }^{11} \mathrm{C}$ data .102

Figure A1-1. Comparisons of seed germination rates under different heavy metal treatments within the first three days

Figure A1-2. Comparisons of root elongation under different heavy metal treatments

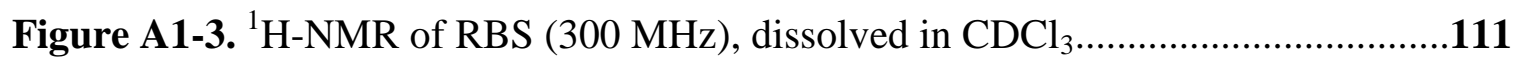

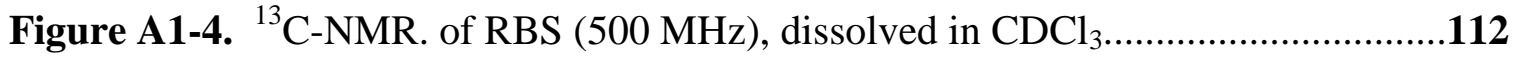

Figure A1-5. Fluorescence Spectra of RBS with different concentrations of $\mathrm{Hg}^{2+} \ldots \ldots .113$

Figure A1-6. Comparison of shoot growth of the opt3-2, irt1-1 mutants and the wild type Arabidopsis with various concentrations of $\mathrm{Pb} / \mathrm{Hg}$ treatments

Figure A1-7. Comparison of the root growth for the opt3-2, irt 1-1 mutants and the wild type Arabidopsis under various concentrations of $\mathrm{Pb} / \mathrm{Hg}$ treatments

Figure A1-8. Illustration of load leaf incubation in the $\left[{ }^{35} \mathrm{~S}\right]$-glutathione containing solution 116

Figure A1-9. ${ }^{35}$ S-glutathione uptake through leaf administration and distribution patterns in the opt3-2 mutant (right) and the WT (left) 
Figure A2-1. Carbon fixation in the three starch mutants and the wild type Arabidopsis

Figure A3-1. Total ${ }^{11} \mathrm{CO}_{2}$ fixation. The experiments were conducted both in the morning (A) and afternoon (B)

Figure A3-2. ${ }^{11} \mathrm{C}$-sugar partitioning in the leaves of three types of Arabidopsis seedlings (Fructose)

Figure A3-3. ${ }^{11} \mathrm{C}$-sugar partitioning in the leaves of three types of Arabidopsis seedlings (Glucose) .135

Figure A3-4. ${ }^{11} \mathrm{C}$-sugar partitioning in the leaves of three types of Arabidopsis seedlings (Sucrose) 


\section{List of Schemes}

Page

Scheme A1-1. Structure of RBS 109

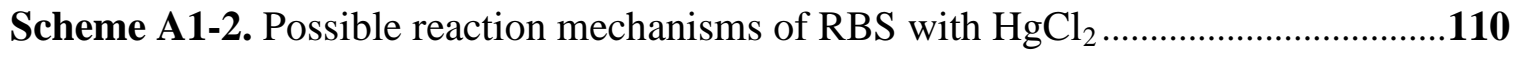




\section{List of Equations}

Page

Equation 3-1: Sucrose synthesis and degradation .........................................................37

Equation 3-2: Biological functions of the enzyme ADP-glucose pyrophosphorylase (AGP) and phosphoglucomutase (PGM). 


\begin{abstract}
A radioactive tracer (radiotracer) is generally defined as a radioactive isotope that is used as a tracer, which can be followed or tracked within a system of interest. The use of radiotracers involves the substitution of a radioactive isotope for one of the naturally occurring isotopes of a particular element. Radiotracers have a wide range of application due to two unique features: a high level of detection sensitivity, and an ability to integrate into living systems. These features make the use of radiotracers particularly useful for studying the dynamic processes that comprise metabolic activity. This work focuses on the use of radiotracers to identify and follow specific biological pathways to facilitate our understanding of plant biology. All of the plants used in this work are Arabidopsis thaliana plants (wild type, col-0 and its different mutants).

Plants are the most common living organisms on earth and have critical roles for the global environment, including human societies. Plants are able to reduce the problem of pollutions, such as uptake contaminants from soils and waters. Also, plants are the basic food producers for other living organisms, which are not only the ones above ground, but also belowground in the rhizosphere (microorganisms). Therefore, if the plant systems are disturbed, it could affect the ecosystem dramatically. In this dissertation, we are trying to use radiotracers to explore the mechanisms of the basic physiology and metabolisms in plants.
\end{abstract}

In Chapter 2, heavy metal uptake and accumulation in one of the Arabidopsis mutant, opt3-2 mutant, was investigated in order to understand the possible mechanisms of phytoremediation. The results showed that the opt $3-2$ mutant plants accumulate $\mathrm{Pb}^{2+}$ in 
both influorescence stems and rosette leaves compared to the wild type (WT). In addition, the results from the radioactive ${ }^{203} \mathrm{~Pb}$ uptake assays indicated that the heavy metal accumulation phenotype in the opt3-2 mutant plants is not due to a kinetic rapid uptake, but to a long-term regulation.

In Chapter 3, the carbon metabolism and translocation in Arabidopsis plants were investigated using both ${ }^{12} \mathrm{C}$ and ${ }^{11} \mathrm{C}$ methods to explore the dynamics of carbon flux in plants, as well as diurnal effects on carbon flux. Three starch mutants (sex $1-1, \operatorname{adg} 1-1$ and pgm-1) and the WT Arabidopsis were used in this study. The results showed that starch regulation is essential not only for plant growth, but also affects sugar metabolism (fructose, glucose and sucrose), carbon allocation to plant roots, and root exudation.

In Chapter 4, the relationship between Fe status and carbon metabolism was discussed. Both ${ }^{12} \mathrm{C}$ and ${ }^{11} \mathrm{C}$ methods were used in this study to investigate the carbon metabolism and translocation in Arabidopsis plants. Two iron-transport mutants (opt3-2 and irt 1-1 mutants) and the WT Arabidopsis were used for this study. The results showed that the Fe status in plants affects the carbon fixation ability in plant leaves, and also alters carbon partitioning (possibly through the production of more organic acids under Fe deficient stress), carbon allocation, and root exudation in plants. Diurnal effects on carbon metabolism and allocation were observed in this study, as well. 


\section{Chapter 1: Introduction}

\subsection{General Concepts of Radiotracer}

A radioactive tracer (radiotracer) is generally defined as a radioactive isotope used as a tracer, which can be followed or tracked in a system of interest. The use of radiotracers involves substituting a radioactive isotope for one of the naturally occurring isotope of a particular element (this could be chemically bound or isolated as an ion). Radiotracers are used in a wide range of areas, such as the basic sciences, medical sciences and in industry. For example, radiotracers are commonly used in pharmaceutical research to monitor drug metabolism in vivo. Radiotracers can also be used as diagnostic reagents to locate cancerous tumors in vivo or therapeutic reagents to kill the cancerous cells. This dissertation will thoroughly discuss how radiotracers can identify or follow specific biological pathways to aid in understanding plant biology.

Radioactive isotopes spontaneously decay through different modes to form more stable nuclei. There are several major decay modes: alpha decay, beta decay, positron decay, electron capture decay and gamma decay.

Alpha $(\alpha)$ decay occurs among the elements with higher atomic numbers, especially with $Z>82$. In this decay mode, an $\alpha$ particle $\left(\mathrm{He}^{2+}\right)$ is emitted from the radioactive nucleus. Alpha particles cannot travel far due to their large size and high charge. Alpha particles lose energy to the surrounding matters through ionizing interactions, which can cause damage to the tissues. Thus, $\alpha$-emitting radioisotopes are not generally used in radiotracer studies. 
Beta $\left(\beta^{-}\right)$decay generally occurs in nuclides with an excess of neutrons. $\beta^{-}$ particles (or negatrons) are high energy electrons emitted from the nucleus, which can travel further than $\alpha$ particles due to their lower mass. The ability of $\beta^{-}$particles to penetrate materials makes them easier to detect by radiation detectors, such as liquid scintillation detectors or gas ionization detectors.

Positron $\left(\beta^{+}\right)$decay occurs in proton-rich nuclides. $\beta^{+}$particles are equal and opposite to $\beta^{-}$particles (e.g., they have the same mass as $\beta^{-}$particles, but are positively charged). A $\beta^{+}$particle is the antiparticle of an electron and will annihilate with a nearby negatively charged electron to form two coincident $511 \mathrm{keV}$ photons whose paths are approximately $180^{\circ}$ apart from each other.

Another type of decay that often occurs in proton-rich nuclides is electron capture decay, which is a decay mode in which one of the inner orbital electrons interacts with the nucleus, combining with a nuclear proton to form a neutron. X-rays and Auger electrons are often emitted following electron capture decay as a consequence of the rearrangement of the orbital electrons.

In gamma $(\gamma)$ decay, the nucleus releases its excess excitation energy by the emission of a photon(s). This decay mode can occur along with other types of decay modes or by itself. For example, a metastable isotope decaying to its ground state via isomeric transition will only emit $\gamma$-rays. 


\subsection{Techniques used for Radiation Detection}

Because radioactive nuclei in radiotracers decay by emitting different types of radiation (particles and/or photons), choosing the appropriate detection techniques is essential to accurately quantify or demonstrate the distribution of the radiotracers in the system(s) of interest.

\section{Common Radiation Detectors}

To quantify the amount of radiotracers present, counting the activity of a sample (such as plant tissues) is generally used. There are several types of radiation detectors, which make use of different detection mechanisms. Gas ionization detectors and scintillation detectors are commonly used radiation detectors.

Gas ionization detectors take advantage of the ionizing effect of radiation on gasses, which subsequently generates electrical current that is used as a signal indicating the radiotracer activity. The Geiger-Muller (G-M) detector is a widely used gas ionization detector, which is highly efficient in detecting $\alpha$ and $\beta^{-}$particles but only $1 \%$ efficient in detecting $\gamma$ rays due to their high penetrating ability through low density gases. The main difficulty in detecting $\alpha$ or low-energy $\beta^{-}$radiation is the extent of absorption occurring in the detector window.

In scintillation detectors, a portion of the energy of ionizing radiation is transferred to fluor atoms or molecules (which can give rise to fluorescence) in a crystalline solid or liquid cocktail. The absorbed energy causes excitation of orbital electrons in the fluor. De-excitation gives rise to the emission of the absorbed energy as 
electromagnetic radiation in the visible or near ultraviolet region (scintillation). Although observing weak scintillations visually is possible, this is usually not a feasible detection method. In general, the photons generated in the scintillation process are converted to photoelectrons and then amplified to form a detectable electrical pulse using a photomultiplier. Therefore, during this process, the original radiation energy is transformed into a measurable pulse. Scintillation detectors generally have two types, solid scintillation (SS) and liquid scintillation (LS). The commonly used SS detector is the $\mathrm{NaI}(\mathrm{Tl})$ detector, which has a high efficiency for $\gamma$ detection. The LS detector uses a solvent cocktail (contains fluor molecules), which directly interacts with the sample. Because the cocktail and sample are in contact with one another, energy is not lost as much as that in the SS detectors by the ionizing radiation while passing through the detector window as in the SS detector, which makes it more efficient for $\alpha$ and $\beta^{-}$ particles, especially low energy $\beta^{-}$particles (e.g., $\beta^{-}$particles emitted from biologically

useful isotopes like ${ }^{3} \mathrm{H},{ }^{14} \mathrm{C}$, and ${ }^{32} \mathrm{P}$ ). However, color and chemical quenching of the cocktail solutionmust be considered (i.e., dye molecules might absorb the fluorescence signal from the fluor molecules in the cocktail and reduce the signal levels; chemicals such as nitromethane might absorb the energy as heat and reduce the signal).

\section{Imaging Instruments}

To visualize the distribution of the radiotracer in vivo of an intact living organism or tissue, radiation imaging techniques are used, such as autoradiography, Single Photon Emission Computed Tomography (SPECT) and Positron Emission Tomography (PET). 
Autoradiography normally uses a plate or film coated with special chemicals that can react with ionizing radiation and subsequently indicate where the radiotracer is distributed. The photographic film emulsion (silver halide) is the classical technique for in vitro localization studies for planar objects. Digital radiography (reading the photographic film with a digital scanner) has developed to not only locate the radioactive sites (qualitative analysis), but also quantify the amount of activity using image analysis software (e.g., Image Quant TL software). A phosphor imaging (PI) screen is similar to the film used in autoradiography, but coated with photo-stimulable phosphor crystals, barium fluorobromide containing a trace amount of bivalent europium as a luminescence center (formulated by FujiFilm company as BaFBr: $\mathrm{Eu}^{2+}$ ) [1]. The crystal can trap and store the energy of the ionizing radiation until scanned by a laser beam, which releases the energy as luminescence. This phosphor technology was first used for medical X-ray diagnoses and later found to be useful for other radioisotope detection in scientific studies, such as ${ }^{11} \mathrm{C},{ }^{14} \mathrm{C},{ }^{35} \mathrm{~S},{ }^{59} \mathrm{Fe}$ and ${ }^{203} \mathrm{~Pb}$. Besides X-rays, the PI screen can also be used for $\beta^{-}$and $\beta^{+}$detection.

SPECT cameras (gamma camera) are rotating scintillation detectors used to acquire tomographic projection data, which are then reconstructed into 3D images. SPECT is sensitive to samples with $\gamma$ radiation. PET cameras are detector rings made of scintillation crystals and photomultipliers to detect pairs of $\gamma$-rays generated by the annihilation of positions (emitted from the radiotracer). SPECT and PET have improved signal-to-noise ratio over autoradiography, and are able to provide quantitative information for stationary or dynamic studies without damaging the sample. Both SPECT 
and PET are often combined with computed tomography (CT) or magnetic resonance imaging (MRI) techniques to give both anatomic and distribution information.

\subsection{Advantages and Disadvantages of Radiotracer Studies}

One of the advantages of radiotracer studies is the high sensitivity of detection, which far exceeds the detection limits of most other chemical or physical methods. Because the detection is based on radiations from every atom, decent signals can be achieved even with very low concentrations ( $\mathrm{pM}$ or $\mathrm{nM}$ scale) of radiotracers present in the system. This facilitates the study of metabolic substances that are normally present in biological tissues at such low concentrations as to defy the most sensitive chemical methods of identification (e.g., tracing the distribution of micronutrients in plants).

Another advantage of radiotracer studies is the possibility to coordinate the dynamic processes within living systems with changes in metabolism. For example, both metal uptake and distribution, and carbon metabolism and allocation in plants could only be approached indirectly before the advent of radiotracer methods. Unfortunately, the fact that radiotracer studies commonly involve such complex dynamic conditions often makes it difficult to interpret the experimental results.

Despite the advantages mentioned above, radiotracers studies also have their own issues that need to be addressed, such as radiation detection, radiation safety and, most importantly, the experimental design.

\subsection{Underlying Assumptions in Design of Radiotracer Experiments}

\subsubsection{Basic Assumptions}


Results of radiotracer experiments will be valid only if the following assumptions are true.

1) There is no significant isotope effect, which means a radioactive isotope behaves chemically and physically identical to the stable isotopes of the same element [2]. It is not exactly true because the difference in masses between radiotracer nuclei and stable nuclei (such as isotopes of hydrogen) can cause a shift in the reaction rate or equilibrium (i.e., the isotope effect) [3]. However, in most cases (elements with a larger mass number than 25), the isotope effect does not significantly affect the actual results. The isotope effect of radioactive isotopes needs to be evaluated before the actual radiotracer experiment.

2) There is no significant radiation damage to the experimental system. It is essential that radiation from the radiotracer does not interfere with the normal biological activities in the system of interest and subsequently distort the actual experimental results. A minimum of radioactivity necessary for a reasonable counting rate is preferred in the radiotracer experiments, especially for $\alpha$ - or $\beta^{-}$- emitting tracers, to avoid damaging the biological systems.

3) There is no deviation from the normal physiological state. In order to reach the minimum activity required in the experiment for detection, the concentration of the administered radiotracer could exceed the normal physiological levels of this compound in the biological system and make the results open to question. Therefore, the specific activity of the radiotracer should be high enough so that the concentration of the radiotracer administered to the system can be within the normal range. 


\section{4) The chemical form of the radioisotope-labeled compound is identical to the}

unlabeled variety. Reactor production of radioisotopes often results in side reactions, which can cause impurities (isotopic impurity or radiochemical impurity) in the radiolabelled tracers administered to experimental systems. The impurities may interfere with the actual radiotracer and affect the results. Therefore, it is important to take the purity of the radiotracers into consideration.

5) Only the labeled atoms are traced. The radioactivity traced in the radiotracer experiment is based on the labeled atoms, not necessarily the intact radiotracer compound administered. The radiotracer compound could be involved in metabolic processes and subsequently cleaved to form other intermediate metabolites.

\subsubsection{Evaluation of the Feasibility of Radiotracer Experiments}

It is critical to carefully design the radiotracer experiment and generate minimum amounts of disposable radioactive waste. Several key factors need to be considered to ensure the feasibility of a radiotracer experiment.

1) Availability of the radiotracer. A primary concern is if a radioisotope of the element to be traced is available with the useful nuclear properties (i.e., half-life and particle energy). A second concern is if the radiotracer compound needed is commercially available or can be easily obtained.

2) Limits of detection. The dose of administered radiotracer compound can be diluted by the experimental system, or only a fraction of the administered compound is taken up. Both situations affect the ability to detect and quantify the amount of the tracer 
present in the system. Thus, the amounts of administered radiotracer present at time points after administration in the experimental system need to be evaluated prior to determining the final protocol for the radiotracer experiments.

3) Evaluation of Hazard. The primary hazard concern is the possibility of harm to the experimenter or co-workers. The hazard from direct external radiation can pose a serious problem if proper shielding is not used. The physical form of the radiotracer (i.e., volatile liquid or solid powder) sometimes requires extra safeguards because it can be potentially inhaled or ingested. The radioactive waste disposal protocol might require modification if the radiotracer experiments are conducted in a biological system that might make the waste bio-hazardous. Additionally, the non-radioactive tracer might be toxic (e.g., $\mathrm{Hg}$ ions), which can make it more difficult to handle if the radioactive isotope is incorporated for a radiotracer study.

\subsection{History of Plant Biological Studies using Radioactive Isotopes}

Using radiotracers to study plant systems stems back to 1923 when George de Hevesey first developed radiotracer methods and later used radioactive $\mathrm{Pb}$ to study $\mathrm{Pb}$ uptake and translocation in plants [4]. With the production of artificially produced radioactive isotopes $\left({ }^{11} \mathrm{C},{ }^{13} \mathrm{~N},{ }^{15} \mathrm{O},{ }^{18} \mathrm{~F}\right)[5-8]$ and the stable isotopes $\left({ }^{15} \mathrm{~N},{ }^{13} \mathrm{C}\right.$ and $\left.{ }^{18} \mathrm{O}\right)$, the radiotracer method was gradually recognized and used in many research fields, such as plant biology. In 1938, Martin Kamen and Sam Ruben were studying the carbon metabolic pathway in photosynthesis by incorporating the short-lived radioisotope ${ }^{11} \mathrm{C}$ into their experiments [9]. After Martin discovered another carbon isotope ${ }^{14} \mathrm{C}$ using a cyclotron, ${ }^{14} \mathrm{C}$ was used as a radiotracer to study particular metabolic pathways in plant 
photosynthesis [10-13]. Kamen and Ruben used ${ }^{14} \mathrm{C}$ to investigate "dark fixation" in plant photosynthesis. Also, Melvin Calvin and his colleagues, using ${ }^{14} \mathrm{C}$, mapped the complete route that carbon travels through a plant during photosynthesis, starting from its absorption as atmospheric carbon dioxide to its conversion into carbohydrates and other organic compounds [10]. Nowadays, with the increasing availability of radioisotopes, commercially available radiolabeled compounds, and better radiation detection techniques, radiotracer methods have become more useful to plant biologists for investigating the basic mechanisms of nutrient metabolism and translocation in plants. Radiotracer experiments are also being used as complimentary evidence to traditional non-radioactive experiments.

\subsection{Radiotracer Studies in This Dissertation}

This work uses radiotracers to investigate basic nutrient metabolism and translocation in plants using radiotracers in Arabidopsis thaliana.

Arabidopsis is a member of the mustard family, which includes cultivated species such as cabbage and radish. Although Arabidopsis is not of major agronomic significance, it offers important advantages for basic research in genetics and molecular biology [14]. For example, Arabidopsis has a rapid growth cycle (6 weeks from germination to reproduction), prolific seed production and is easy to cultivate. Also, Arabidopsis has a small genome that has been sequenced, can be transformed easily by agrobacterium, and a large number of mutant plants are commercially available. Such features make Arabidopsis a good model for plant biological studies. Arabidopsis has 
different ecotypes. Columbia-O (col-O) is used as a control (wild type, WT) in all the studies mentioned in the dissertation.

Below is an outline of the radiotracer studies discussed in this dissertation and the radiotracers used for the studies.

Chapter 2 describes a study of heavy metal accumulation in the opt3-2 mutant (which was shown to over-accumulate Fe and several other di-valent cations in aerial tissues) and the WT Arabidopsis. ${ }^{203} \mathrm{~Pb}^{2+}$ was used to investigate uptake behavior and distribution patterns in the mature opt3-2 mutant and WT plants. ${ }^{203} \mathrm{~Pb}^{2+}$ was purchased from Lantheus Medical Imaging and came dissolved in $0.5 \mathrm{M} \mathrm{HNO}_{3}$.

Chapter 3 describes a study of carbon metabolism and allocation in three starch mutant plants, sexl-1 (starch excess), adgl-1 and pgm-1 (starch deficient), and WT plants. ${ }^{11} \mathrm{CO}_{2}$ was used to explore the short-term carbon flux in the starch mutant plants. ${ }^{11} \mathrm{CO}_{2}$ was produced by the cyclotron in the Brookhaven National Laboratory (BNL).

Chapter 4 describes a study of how carbon metabolism and allocation are affected by the external and internal iron status in Arabidopsis mutants (opt3-2 and irt11) compared to the WT plants. In this study, ${ }^{59} \mathrm{Fe}^{3+}$ and ${ }^{11} \mathrm{CO}_{2}$ were both used to investigate the dynamic relationship of carbon metabolism and Fe status within a short time window. ${ }^{59} \mathrm{Fe}^{3+}$ was purchased from the Perkin Elmer and dissolved in $0.5 \mathrm{M} \mathrm{HCl}$. ${ }^{11} \mathrm{CO}_{2}$ was produced by the same method mentioned in Chapter 3. 


\section{References}

1. $\quad$ http://www.sb.fsu.edu/ xray/Manuals/ip.pdf.

2. Choppin, G.R., http://oregonstate.edu/instruct/ch374/ch418518/Chapter\%20IV\%20Radiotracers.pdf. Nuclear chemistry. 2nd edition.

3. Lanigan, G.J., et al., Carbon isotope fractionation during photorespiration and carboxylation in Senecio. Plant Physiol, 2008. 148(4): p. 2013-20.

4. Hevesy, G., The absorption and translocation of lead by plants. A contribution to the application of the method of radioactive indicators in the investigation of the change of substance in plants. Biochemistry Journal, 1923. 17: p. 7.

5. http://www.nasonline.org/publications/biographical-memoirs/memoir-pdfs/lauritsencharles.pdf.

6. Ferrieri, R.A., et al., On-line production of $13 \mathrm{~N}$-nitrogen gas from a solid enriched $13 \mathrm{C}$ target and its application to $13 \mathrm{~N}$-ammonia synthesis using microwave radiation. The International Journal of Applied Radiation and Isotopes, 1983. 34(6): p. 897-900.

7. Fowler, J.S., Wolf, A.P., Synthesis of carbon-11, fluorine-18, and nitrogen-13 labeled radiotracers for biomedical applications, in Other Information: Portions of document are illegible. 1981. p. Medium: ED; Size: Pages: 124.

8. Ido, T., et al., Labeled 2-deoxy-D-glucose analogs. 18F-labeled 2-deoxy-2-fluoro-Dglucose, 2-deoxy-2-fluoro-D-mannose and 14C-2-deoxy-2-fluoro-D-glucose. Journal of Labelled Compounds and Radiopharmaceuticals, 1978. 14(2): p. 175-183.

9. Ruben, S., et al., Photosynthesis with Radio-Carbon. Science, 1939. 90(2346): p. 570571.

10. Calvin, M., Benson, A. A., The Path of Carbon in Photosynthesis. Science, 1948. 107(2784): p. 476-480.

11. Gest, H., Samuel Ruben's Contributions to Research on Photosynthesis and Bacterial Metabolism with Radioactive Carbon. Photosynth Res, 2004. 80(1-3): p. 77-83. 


\section{Chapter 1}

12. Ruben, S., Kamen, M. D., Photosynthesis with Radioactive Carbon. IV. Molecular Weight of the Intermediate Products and a Tentative Theory of Photosynthesis. Journal of the American Chemical Society, 1940. 62(12): p. 3451-3455.

13. Ruben, S., Kamen, M. D., Long-Lived Radioactive Carbon: C-14. Physical Review, 1941. 59(4): p. 349-354.

14. http://www.arabidopsis.org/portals/education/aboutarabidopsis.jsp. 


\section{Chapter 2: Accumulation of Heavy Metals by the Arabidopsis opt3-2 Mutant}

\subsection{Isotope Summary}

\begin{tabular}{|c|c|c|c|}
\hline Isotopes & Half-life & Decay Mode & E \\
\hline${ }^{203} \mathrm{~Pb}$ & $51.9 \mathrm{~h}$ & $\mathrm{EC}(100 \%), \gamma$ & $0.975 \mathrm{MeV}(\mathrm{EC}), 0.279 \mathrm{MeV}(\gamma, 81 \%)$ \\
\hline
\end{tabular}

\subsection{Background}

Metals are naturally present in the Earth's crust at various levels [1]. Mining, industry, and agriculture lead to accelerated release of metals into ecosystems, causing serious environmental problems as well as posing a threat to human health [2]. Although many metals are essential for cells (e.g., $\mathrm{Cu}, \mathrm{Fe}, \mathrm{Mn}, \mathrm{Ni}, \mathrm{Zn}$ ), all metals are toxic at higher concentrations [3]. More than 50,000 metal-contaminated sites await remediation in the U.S. alone. Approximately $80 \%$ of U.S. Superfund sites (designated by the U.S. Environmental Protection Agency as priority sites for cleanup) contain heavy metals, often mixed with organic pollutants [4].

Heavy metal contamination in the environment, if not carefully treated, can be harmful to biological systems since heavy metals are not biodegradable and can accumulate in living organisms such as plants, which are at the low end of the food chain. If consumed as daily food, heavy metals could easily reach toxic levels in animals and humans. Heavy metal contamination also poses dangers to the areas surrounding the contaminated sites due to possible migration as dust, leachates through soil, or untreated 
sewage sludge. There are various methods to clean up heavy metal contaminated sites and reduce the levels of toxicity, but most of them are costly, do not reach their optimum performance, and also no living organisms can use the soil during the remediation process.

Using a natural bio-process to clean up contaminated sites would be more ideal. Phytoremediation is not a new concept $[2,5-10]$, but recently, has been revisited due to its efficiency, affordable cost and most importantly environmental friendliness. Phytoremediation involves using plants to clean up the contaminated environments by removing, containing or transforming hazardous contaminants, such as heavy metals, to improve environmental quality. Research has shown that certain plant species have unique and selective uptake capabilities for heavy metals in their root systems and have translocation, bioaccumulation, and detoxification abilities through the whole plant body system [8, 11-15]. These features make plants a promising alternative for environmental remediation.

Previous research has shown that Arabidopsis oligo-peptide transporter3-2 (opt32) mutant plants were able to over-accumulate Fe and some other di-valent cations, such as $\mathrm{Mn}, \mathrm{Zn}$ and $\mathrm{Cu}$, due to the constitutively up-regulated expression of iron-regulated transporter 1-1 (IRT1-1) [16, 17]. IRT1-1 is an efficient iron transporter found in Arabidopsis roots. IRT1 not only can transport $\mathrm{Fe}^{2+}$ into the plant root system, but also can transport certain di-valent cations, such as Mn, Zn, Cd, etc. [18, 19]. All of the features mentioned above suggest that IRT1-1 could play a role in transporting heavy metals, and likely explains the over-accumulation of these metals in the tissues of the opt3-2 mutant. This makes the opt3-2 Arabidopsis mutant a model system for studying 
the general mechanisms of heavy metal accumulation in plants, which could be later translated to better plant systems (e.g., poplar trees) for phytoremediation purposes.

In this study, inductively-coupled plasma mass spectroscopy (ICP-MS) and radioactive tracer $\left({ }^{203} \mathrm{~Pb}^{2+}\right)$ assays were used to investigate heavy metal accumulation in the tissues of the opt3-2 Arabidopsis mutant, as well as their uptake behavior and distribution patterns. Transport assays using yeast were also used to further explore the possible accumulation mechanism.

\subsection{Materials and Methods}

\subsubsection{Materials}

Bleach used for seed sterilization was purchased from Pure Bright (Columbia, MO). Premier Pro-Mix soil was purchased from Quakertown, PA, USA. The restriction enzyme HindIII was purchased from New England BioLabs (Ipswich, MA). Difco ${ }^{\text {TM }}$ Yeast Nitrogen Base (without amino acids) was purchased from Becton, Dickinson and Company (Franklin Lakes, NJ). Triton X-100, Murashige and Skoog (MS) salts with vitamins, $\mathrm{HgCl}_{2}$, casamino acids, L-tryptophan, adenine and agar were purchased from Sigma (St. Louis, MO). Sucrose, $\mathrm{Pb}(\text { acetate })_{2}$ and MES (2-(N-morpholino)ethanesulfonic acid, monohydrate) were purchased from Fisher Scientific (St. Louis, MO).

${ }^{203} \mathrm{~Pb}(\mathrm{NO} 3)_{2}$ was purchased from Lantheus Medical Imaging (N. Billerica, MA).

\subsubsection{Plant Growth Conditions}

Seeds were surface-sterilized with a $35 \%$ bleach solution containing $0.1 \%$ Triton $\mathrm{X}-100$ for $20 \mathrm{~min}$ and then rinsed several times with sterile water. Prior to germination seeds were placed in the cold room $\left(4^{\circ} \mathrm{C}\right)$ for $2-3$ days to align the seedling germination 
times. For routine growth, seedlings were grown in agar medium containing one-halfstrength Murashige and Skoog (MS) salts, MS vitamins, $1 \%$ sucrose (w/v), $0.05 \%$ MES. The $\mathrm{pH}$ of the medium was adjusted to 5.7 with $5 \mathrm{M} \mathrm{NaOH}$ solution (conc?). For metal uptake assays, one-week-old seedlings were transferred to the medium with different concentrations of $\mathrm{Pb}(\text { acetate })_{2}(500 \mu \mathrm{M}, 750 \mu \mathrm{M})$ or $\mathrm{HgCl}_{2}(10 \mu \mathrm{M}, 20 \mu \mathrm{M})$. For root analysis, petri dish plates were placed vertically. For seed amplification, two-week-old seedlings were transferred and grown in Premier Pro-Mix soil. For ICP-MS analysis, plants were grown under the same conditions as used for seed amplification except that the soil was treated with $\mathrm{Pb}$ or $\mathrm{Hg}$ salts. For $\mathrm{Pb}$ treatment, $500 \mu \mathrm{M} \mathrm{Pb}$ (acetate) ${ }_{2}$ solution was used to water the plants during the growth period. For $\mathrm{Hg}$ treatment, the soil was premixed with $\mathrm{HgCl}_{2}$ salts. Plants were all grown at $22^{\circ} \mathrm{C}$ under $100 \mu$ mole $\mathrm{m}^{-2} \mathrm{~s}^{-}$

${ }^{1}$ fluorescent white light with an $8 \mathrm{hr}$ (day)/16 hr (night) photoperiod. For ${ }^{203} \mathrm{~Pb}^{2+}$ uptake assay, seeds were germinated in sand mix $\left(\right.$ Quikrete $^{\mathrm{TM}}$ all-purpose sand and Sta-Green ${ }^{\mathrm{TM}}$ Vermiculite; sand : vermiculite (v/v) was 5:1; sand was washed with tap water several times, then washed with distilled water 4 times, then mixed with half-strength MS-soaked vermiculite) and grown for 1.5 months. The growth conditions were the same as mentioned above except the photoperiod was $12 \mathrm{hr}$ (day)/12 $\mathrm{hr}$ (night) for this experiment. This photoperiod resulted in plants that grew slower and with larger leaves, which was preferred for the radiotracer experiments. The wild type (WT) Arabidopsis used in this study was Columbia-O (col-0). 


\subsubsection{Determination of Chlorophyll Content}

Plants were grown under the same conditions as mentioned above in the plant growth assay. All the petri dish plates were placed horizontally and the seedlings were grown for 2 weeks in the growth chamber. 5-8 seedlings were selected from both wild type and opt3-2 mutant plants grown under different metal treatments. Each seedling was put into a $1.5 \mathrm{ml}$ Eppendorf tube and the chlorophyll was extracted with $1 \mathrm{ml}$ of $100 \%$ methanol. The extract solutions $(200 \mu \mathrm{l})$ were pipetted into a 96-well plate and measured with a Bio-TEK plate reader at 652, 665 and $750 \mathrm{~nm}$. The chlorophyll content was calculated using the following equations [20]:

$$
\begin{gathered}
\text { Chl } \mathrm{a}=[16.29 \times(\mathrm{A} 665-\mathrm{A} 750)]-[8.54 \times(\mathrm{A} 652-\mathrm{A} 750)] \\
\mathrm{Chl} \mathrm{b}=[30.66 \times(\mathrm{A} 652-\mathrm{A} 750)]-[13.58 \times(\mathrm{A} 665-\mathrm{A} 750)] \\
\mathrm{Chl} \mathrm{a+b}=[22.12 \times(\mathrm{A} 652-\mathrm{A} 750)]+[2.71 \times(\mathrm{A} 665-\mathrm{A} 750)]
\end{gathered}
$$

A652, A655 and A750 indicate the absorbance at 652, 655 and $750 \mathrm{~nm}$, respectively.

Chl a: Chlorophyll a; Chl b: Chlorophyll b; Chl $a+b$ : Chlorophyll a and Chlorophyll b.

\subsubsection{ICP-MS Analysis}

The above-ground tissue of one-month-old pot-grown Arabidopsis plants were harvested and separated into rosette leaves and influorescence stems, and then ground into powders in liquid $\mathrm{N}_{2}$. ICP-MS analysis of $\mathrm{Hg}$ was done at the University of Missouri Research Reactor (MURR). ICP-MS analysis of $\mathrm{Pb}$ was done at the Environmental Molecular Sciences Laboratory (EMSL), Pacific Northwest National Laboratory (PNNL) 


\subsubsection{Radioactive ${ }^{203} \mathrm{~Pb}^{2+}$ Uptake Assay and Imaging}

One-and-a-half month old plants grown in sand/vermiculite mixtures (s/v mix) were used for the ${ }^{203} \mathrm{~Pb}$ uptake assay. Plants were removed from the pot by immersion in water to loosen the s/v mix while keeping the roots intact. The plants were washed gently with tap water and kept in a falcon tube filled with $0.5 \mathrm{mM} \mathrm{CaCl}_{2}$ solution for $15 \mathrm{~min}$ and then transferred to another tube with half-strength MS medium and equilibrated for 6 hours. The experimental set-up is shown in Figure 2-5A. Just before adding $200 \mu \mathrm{Ci}$ of ${ }^{203} \mathrm{~Pb}\left(\mathrm{NO}_{3}\right)_{2}$, non-radioactive carrier $\mathrm{Pb}$ (acetate $)_{2}$ was added into the medium to reach a final concentration of $250 \mu \mathrm{M}$. The medium solution was stirred to uniformly distribute the $\mathrm{Pb}^{2+}$ and the plants were incubated for 24 hours. The roots were rinsed with $0.5 \mathrm{mM} \mathrm{CaCl}_{2}$ solution followed by distilled water to remove any surfaceadhered $\mathrm{Pb}^{2+}$. The plants were dried, weighed and cut into shoots and roots, then counted using a $\mathrm{NaI}(\mathrm{Tl})$ well detector. Individual rosette leaves were cut off, displayed away from the rosette leaf center and then exposed on the phosphor-image screen (FujiFilm), which was later analyzed using a phosphor-imager (Typhoon 900).

\subsubsection{Yeast Transformation and Growth Assays}

The AtIRT1-1 gene was sub-cloned into the yeast expression vector $p D B 20$ (pAtIRT1-1 was obtained from Stacey's Lab). pAtIRT1-1 and vector $p D B 20$ were individually transformed into $S$. cerevicea yeast strain BY4730. The transformed yeast was selected from minus-Uracil (-Ura) medium (means media without uracil). To confirm the transformation, the two plasmids were extracted from $B Y 4730$ yeast, amplified in E. coli and digested with restriction enzyme HindIII. Synthetic Defined 
medium without uracil (SD-Ura) was used, containing $0.67 \%$ Yeast Nitrogen base (without amino acids), $0.1 \%$ casamino acids, $0.01 \%$ tryptophan and adenine. (protocols were obtained from Stacey's lab and attached in Appendix B). One colony of yeast was inoculated in $3 \mathrm{ml}$ of SD-Ura medium and shaken at $180 \mathrm{rpm}$ at $30{ }^{\circ} \mathrm{C}$ overnight (final $\left.\mathrm{OD}_{600}=0.9-1.2\right)$. The cells were centrifuged at $4500 \mathrm{rpm}$ for $6 \mathrm{~min}$ at $25{ }^{\circ} \mathrm{C}$. After decanting the supernatant, the cells were washed three times with sterilized ultrapure $\mathrm{H}_{2} \mathrm{O}$. The cells were re-suspended in ultra-pure $\mathrm{H}_{2} \mathrm{O}$ and added to $3 \mathrm{ml}$ of new SDUra medium to achieve an $\mathrm{OD}_{600}=0.03-0.05$. Yeast was grown in the medium without heavy metals for $\sim 3 \mathrm{hr}$, after which time heavy metal solutions $\left(\mathrm{HgCl}_{2}, \mathrm{~Pb} \text { (acetate }\right)_{2}$, $\mathrm{CdCl}_{2}$ ) of different concentrations were added. The growth of cultures was scored over the period of 20 or 24 hours by measuring the $\mathrm{OD}_{600}$.

\subsubsection{Statistical Analysis}

Data was subjected to the Student t-test for unpaired samples assuming an unequal variance. Statistical significance levels were assigned to the following rating scale $(*, \mathrm{p}<0.05 ; * *, \mathrm{p}<0.01 ; * * *, \mathrm{p}<0.001)$

\subsection{Results}

\subsubsection{Toxic Effects of Heavy Metals on Plant Growth}

Heavy metals are known to be toxic to plants and can significantly inhibit plant growth and physiology, such as shoot/root growth and chlorophyll synthesis [14]. To investigate the response of the opt3-2 mutant relative to the WT, both were grown in media with different concentrations of $\mathrm{Pb}^{2+}$ or $\mathrm{Hg}^{2+}$, and several physiological properties were measured. (Fig. 2-1 and Fig. 2-2) Shoot and root growth of both the opt3-2 mutant 
and the WT plants were inhibited when heavy metal ions were introduced into the medium, especially at the higher concentration of $\mathrm{Pb}^{2+}$ or $\mathrm{Hg}^{2+}$. The shoot growth of the opt3-2 mutant was less than the WT even in the absence of heavy metals. However, in the absence of heavy metals, the chlorophyll content of the mutant and the WT plants were identical. This was not the case when heavy metals were added; the opt3-2 mutant plants showed significantly lower chlorophyll content, which was also observed visually when seedlings were grown in the agar plates. (Fig. 2-1) The root growth of the opt3-2 mutant was similar to the wild type under the control conditions, but was significantly inhibited in the presence of high concentrations of heavy metal ions. Compared to the wild type, the opt3-2 mutant had less root mass, especially fewer lateral roots. (Fig. 2-2)

\subsubsection{Heavy Metal Quantification in Plant Tissues by ICP-MS}

To quantify the amount of heavy metal taken up and stored in the aerial plant tissues, influorescence stems (including leaves, flowers and seliques) and rosette leaves of both opt3-2 mutant and WT plants were ground into powders under liquid nitrogen and analyzed by ICP-MS. Under control conditions, no heavy metals were added to the soil. Therefore, the amount of $\mathrm{Pb}$ or $\mathrm{Hg}$ present in the plant tissues under the control conditions is due to the natural amount of $\mathrm{Pb}$ or $\mathrm{Hg}$ present in the soil or water. Overall,

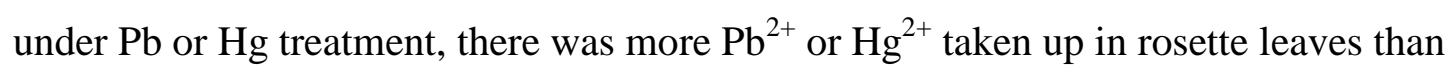
stems in both WT and opt3-2 mutant plants. The opt3-2 mutant showed significantly more $\mathrm{Pb}^{2+}$ in both influorescence and rosette leaves compared to the wild type, which indicates that opt3-2 over-accumulates $\mathrm{Pb}^{2+}$ in the above-ground tissues. (Fig. 2-3a) The $\mathrm{Hg}$ data showed the same general trend as that seen with the $\mathrm{Pb}$ treatment. However, the 
amount of accumulation of $\mathrm{Hg}$ was ten thousand times lower than $\mathrm{Pb}$ and the differences seen were not statistically significant.

Heavy metal uptake in the roots was investigated using radiotracers, not by ICPMS. (Fig. 2-6)

\subsubsection{Radioactive ${ }^{203} \mathrm{~Pb}^{2+}$ Uptake Assay}

Heavy metal uptake in the roots was investigated using radiotracers (Fig. 2-6).

The results from the ${ }^{203} \mathrm{~Pb}^{2+}$ uptake assays showed no significant difference between opt3-2 and WT plants in the total amount of $\mathrm{Pb}$ taken up into the entire plant. There was no apparent over-accumulation of $\mathrm{Pb}$ in the shoots of the opt3-2 mutant plants compared to the WT. Both the opt3-2 mutant and WT plants stored over 90 percent of the $\mathrm{Pb}$ in the roots and only moved a small fraction to the shoots (Fig. 2-6). As shown in the phosphor image (Fig. 2-5), the $\mathrm{Pb}$ in the leaves was intensively localized in small spots, which are possibly the trichomes or the stomata[7]. Further studies are needed to clarify this distinct phenotype.

\subsubsection{IRT1 Does Not Mediate Pb or Hg Uptake}

To investigate the ability of IRT1-1 to transport $\mathrm{Pb}^{2+}$ or $\mathrm{Hg}^{2+}, B Y 4730$ yeast cells was transformed with the $p A t I R T 1-1$. Yeast cells with transformed $p D B 20$ (an empty vector) were used as a negative control. $\mathrm{Pb}^{2+} / \mathrm{Hg}^{2+}$ is toxic to yeast and, therefore, if IRT1-1 does transport these metals, we would assume that yeast expressing this transporter would accumulate more $\mathrm{Pb}^{2+} / \mathrm{Hg}^{2+}$ and their growth would be inhibited significantly more than cells expressing the empty vector. 
Yeast cells were first grown in a medium with $\mathrm{Cd}^{2+}$, a known substrate for IRT1, to test if AtIRT1-1 was expressed and functioning correctly [21]. As shown in Figure 24b, the growth of yeast expressing AtIRT1-1 was inhibited significantly when $\mathrm{Cd}^{2+}$ was added to the medium compared to yeast transformed with $p D B 20$, while in Figure 2-4a both transformed yeast cells grow similarly with normal nutrient medium. These results indicate that the yeast cells are expressing pAtIRT1-1, which is mediating the uptake of $\mathrm{Cd}$. However, in contrast to the results obtained with $\mathrm{Cd}$, there was no apparent difference in growth for the yeast strains under different concentrations of $\mathrm{Pb}^{2+}$ or $\mathrm{Hg}^{2+}$ (Fig. 2-4). Both transformed yeast lines were affected to a similar level with or without pAtIRT1-1. In addition, both yeast transformants exhibited a better tolerance to $\mathrm{Pb}$ than to $\mathrm{Hg}$. Compared to the $\mathrm{Hg}$ treatment, both yeast transformants grew much better even in the presence of the higher concentrations of $\mathrm{Pb}$.

\subsection{Discussion}

Our current results show that compared to wild type Arabidopsis, the opt3-2 mutant over-accumulates $\mathrm{Pb}$ and $\mathrm{Hg}$ in the aerial tissues (Fig. 2-3). This makes the opt3-2 mutant a good system for investigation of the underlying mechanism of heavy metal accumulation in plants, which is very important for developing and improving engineered plants for phytoremediation purposes.

The root growth of opt3-2 mutant plants were more affected by heavy metal treatment than the wild type, although both the opt3-2 mutant and the wild type were inhibited when high concentrations of $\mathrm{Pb}^{2+} / \mathrm{Hg}^{2+}$ were present. Several studies have shown that $\mathrm{Pb}^{2+}$ and $\mathrm{Hg}^{2+}$ are primarily accumulated in plant roots relative to aerial 
tissues and can inhibit root growth (including lateral root growth) dramatically[11, 14, 22]. As seen in Figure 2-2, both the main root and lateral root growth were inhibited when seedlings were grown under high concentrations of $\mathrm{Pb}^{2+} / \mathrm{Hg}^{2+}$, especially opt3-2 mutant plants.

In general, under high concentrations of heavy metals, the shoot growth of both the opt3-2 and wild type was inhibited in terms of shoot mass and chlorophyll content (Fig. 2-1). Hg can be transported into the leaves and it is known to affect photosynthesis and oxidative metabolism by interfering with electron transport in chloroplasts and mitochondria $[23,24]$. Pb may also directly or indirectly affect photosynthesis. As shown in Figure 2-1, the opt3-2 mutant showed significantly lower chlorophyll content under heavy metal treatment, which may be due to a substitution for Fe by the heavy metal to affect the normal chlorophyll synthesis $[25,26]$. The opt3-2 mutant also showed a consistently lower shoot mass than the WT, even in the absence of heavy metal addition. This may be due to the over-accumulation of other metals, such as Fe, which occurs in this mutant even when excess $\mathrm{Pb}$ or $\mathrm{Hg}$ is not present [16].

In the aerial portion of the plants, as shown in Figure 2-3, the major accumulation was observed in rosette leaves for both $\mathrm{Pb}$ and $\mathrm{Hg}$. There was much less $\mathrm{Pb} / \mathrm{Hg}$ present in the stems. This indicates that rosette leaves are sinks for $\mathrm{Pb}^{2+} / \mathrm{Hg}^{2+}$ once they are taken up, immobilized and stored in certain regions of the cell, such as the cell wall and the vacuoles $[7,11,24]$.

The ${ }^{203} \mathrm{~Pb}$ uptake assay showed there was no significant difference in $\mathrm{Pb}$ accumulation between the opt3-2 and the wild type within a 24-hour window. (Fig. 2-6) 
These results are different from the data seen using ICP-MS analysis. The results suggest that the initial rate of metal uptake (measured by ${ }^{203} \mathrm{~Pb}$ uptake) is not affected in the opt32 mutant plants but long term accumulation of heavy metals (as measured by ICP-MS analysis) is strongly affected in the mutant. These data are consistent with the notion that OPT3 does not mediate metal uptake in the roots but rather is involved in internal mobilization of metals within the plant [16]. Most of the studies on heavy metal accumulation only show the cumulative amount of metals in the plant tissues. However, using radiotracer assays to explore what is happening during the accumulation process allowed us to distinguish between short term uptake versus long term accumulation mechanisms.

$\mathrm{Hg}$, unlike $\mathrm{Pb}$, was not shown to be over-accumulated in any of the tissues of the opt3-2 mutant according to the ICP-MS results (Fig. 2-3). However, it is possible that some of the $\mathrm{Hg}$ that was taken up by the plants was transformed into $\mathrm{Hg}^{0}$ through plant biological processes; $\mathrm{Hg}^{0}$ is volatile and easily released into air. This process may result in a loss of $\mathrm{Hg}$ content in both of the WT and the opt3-2 mutant, which could affect the accuracy of ICP-MS analysis.

In the past, the over-accumulation of $\mathrm{Fe}$ and $\mathrm{Cd}$ in opt3-2 mutant plants has been explained by the constitutive, up-regulation of the IRT1 transporter in this mutant [16]. Indeed, IRT1-1, although initially described as an iron transporter, does transport Cd, as well as a few other divalent cations. However, IRT1 is not known to transport either Pb or Hg. In order to test this directly, we expressed AtIRT1 in yeast and measured the effects of heavy metal addition on yeast growth. In control experiments, yeast expressing AtIRT1 showed increased sensitivity to Cd addition, consistent with the expression of a 


\section{Chapter $2=26$}

functional transporter. However, yeast expressing AtIRT1 showed no increased sensitivity to the toxic effects of $\mathrm{Pb}$ or $\mathrm{Hg}$. Therefore, we conclude that up-regulation of AtIRT1 in opt3-2 mutant plants is unlikely to be the explanation for the increased sensitivity and accumulation of $\mathrm{Pb}$ or $\mathrm{Hg}$ by these plants. The analysis of DNA microarray data (unpublished data from David G. Mendoza-Cozatl, data not shown) comparing the opt3-2 mutant to wild type indicates that the expression of a number of transporter genes is affected in the mutant, in addition to AtIRT1. It is possible that one or more of them may be responsible for $\mathrm{Pb} / \mathrm{Hg}$ trafficking. 

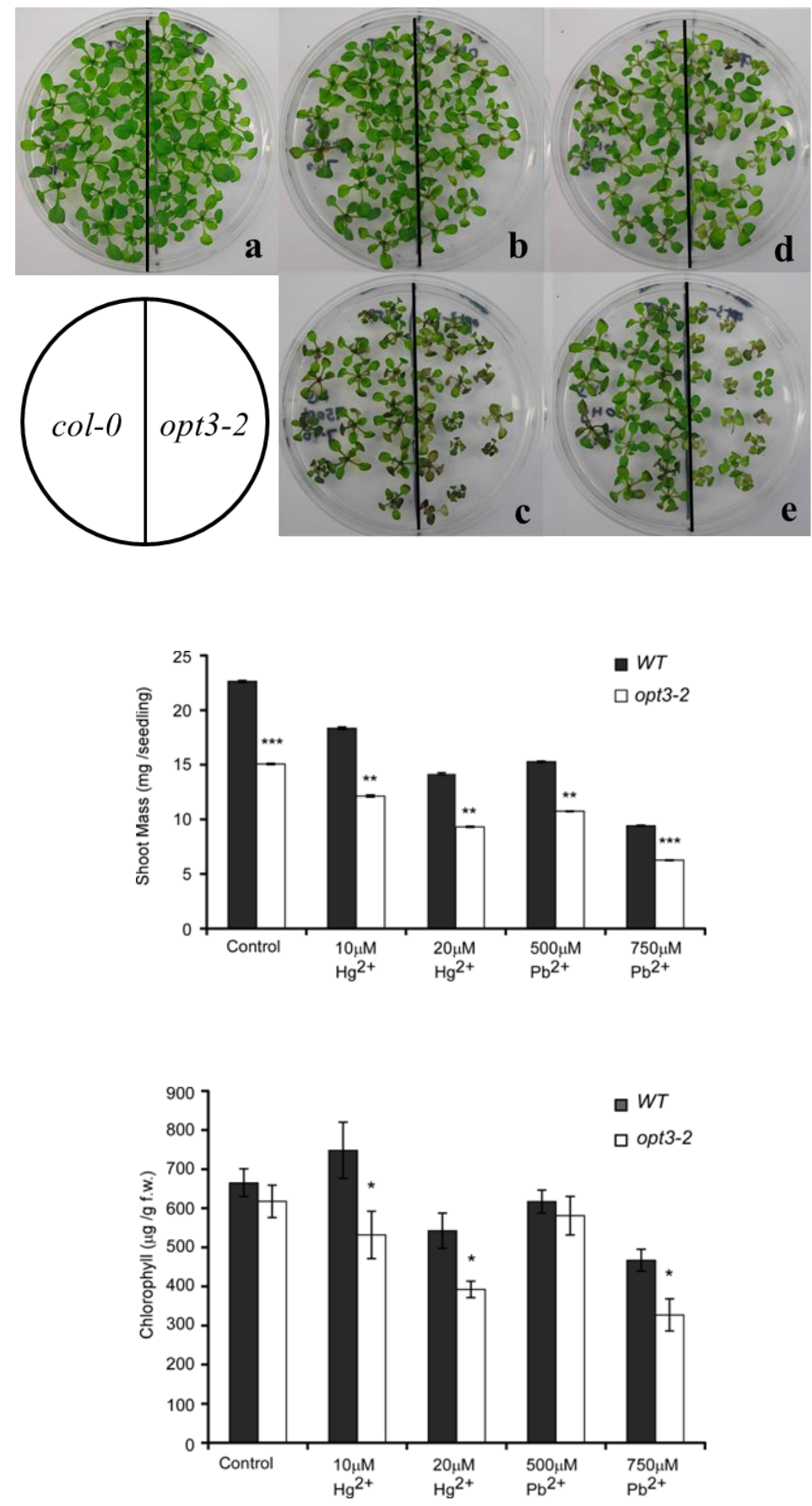

Figure 2-1. Shoot growth in different heavy metal treatments. upper panel: a, without heavy metal; $\mathbf{b}$ and $\mathbf{c}$, with $500 \mu \mathrm{M}$ and $750 \mu \mathrm{M} \mathrm{Pb}$ (acetate) $)_{2}$; d and $\mathbf{e}$, with $10 \mu \mathrm{M}$ and $20 \mu \mathrm{M} \mathrm{HgCl}_{2}$. col-0 is on the left side while opt3-2 is on the right side. Seedlings are two weeks old. Lower panel: shoot mass ( $n=12$ seedlings) and chlorophyll content ( $n=8$ seedlings, mean \pm SE) under different heavy metal treatments. col-O is the wild type Arabidopsis used in all the studies. See Table A1-3 and A1-4 in Appendix A for tabular data. 

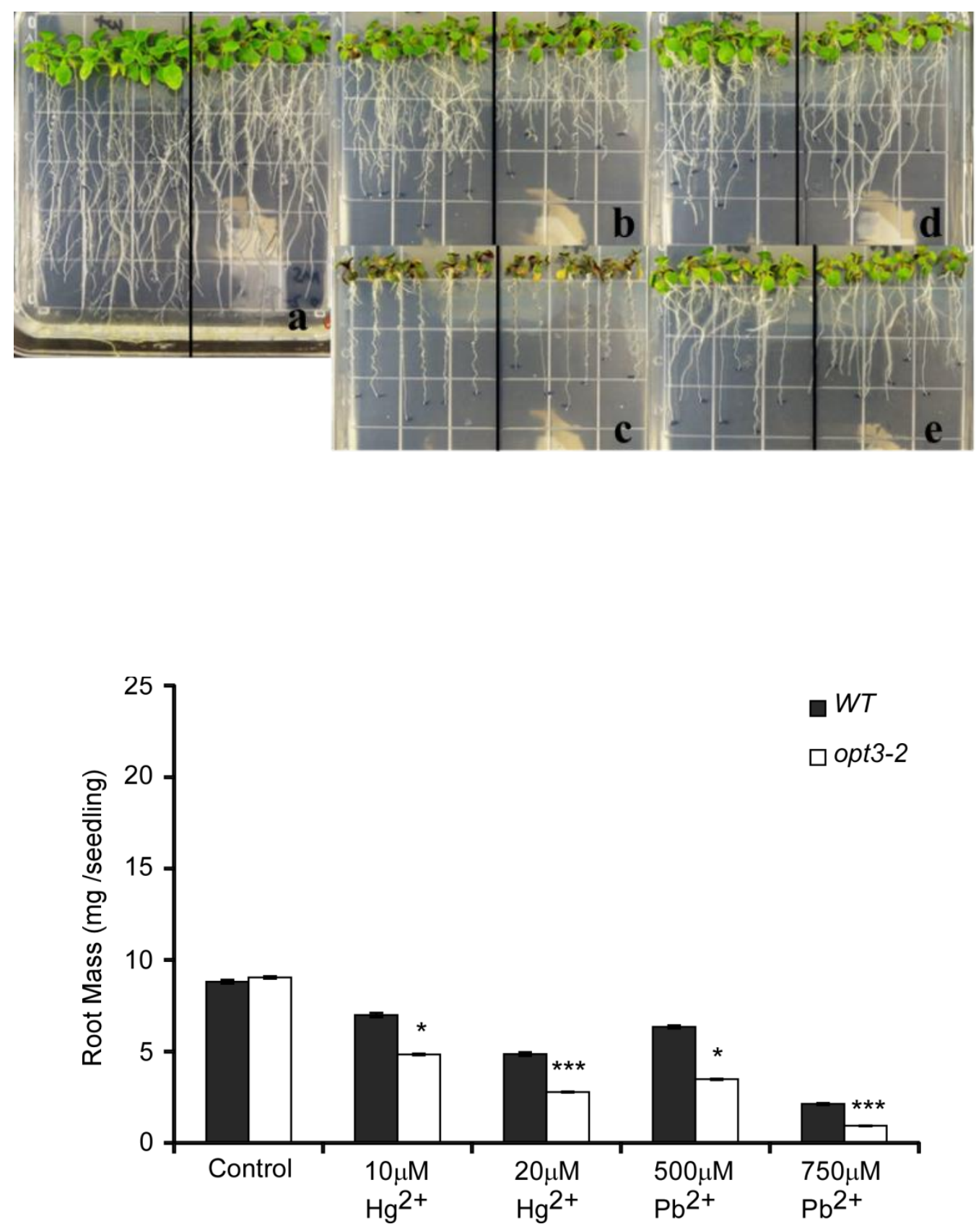

Figure 2-2. Root growth in different heavy metal treatments. Left panel: a, without heavy metal; b and c, with $500 \mu \mathrm{M}$ and $750 \mu \mathrm{M} \mathrm{Pb}$ (acetate) ${ }_{2} ; \mathbf{d}$ and $\mathbf{e}$, with $10 \mu \mathrm{M}$ and $20 \mu \mathrm{M} \mathrm{HgCl}_{2}$. col-0 is on the left side while opt $3-2$ is on the right side. Seedlings are two weeks old. Right panel: Root mass ( $n=12$ seedlings, mean \pm SE) under different heavy metal treatments. See Table A1-3 in Appendix A for tabular data. 

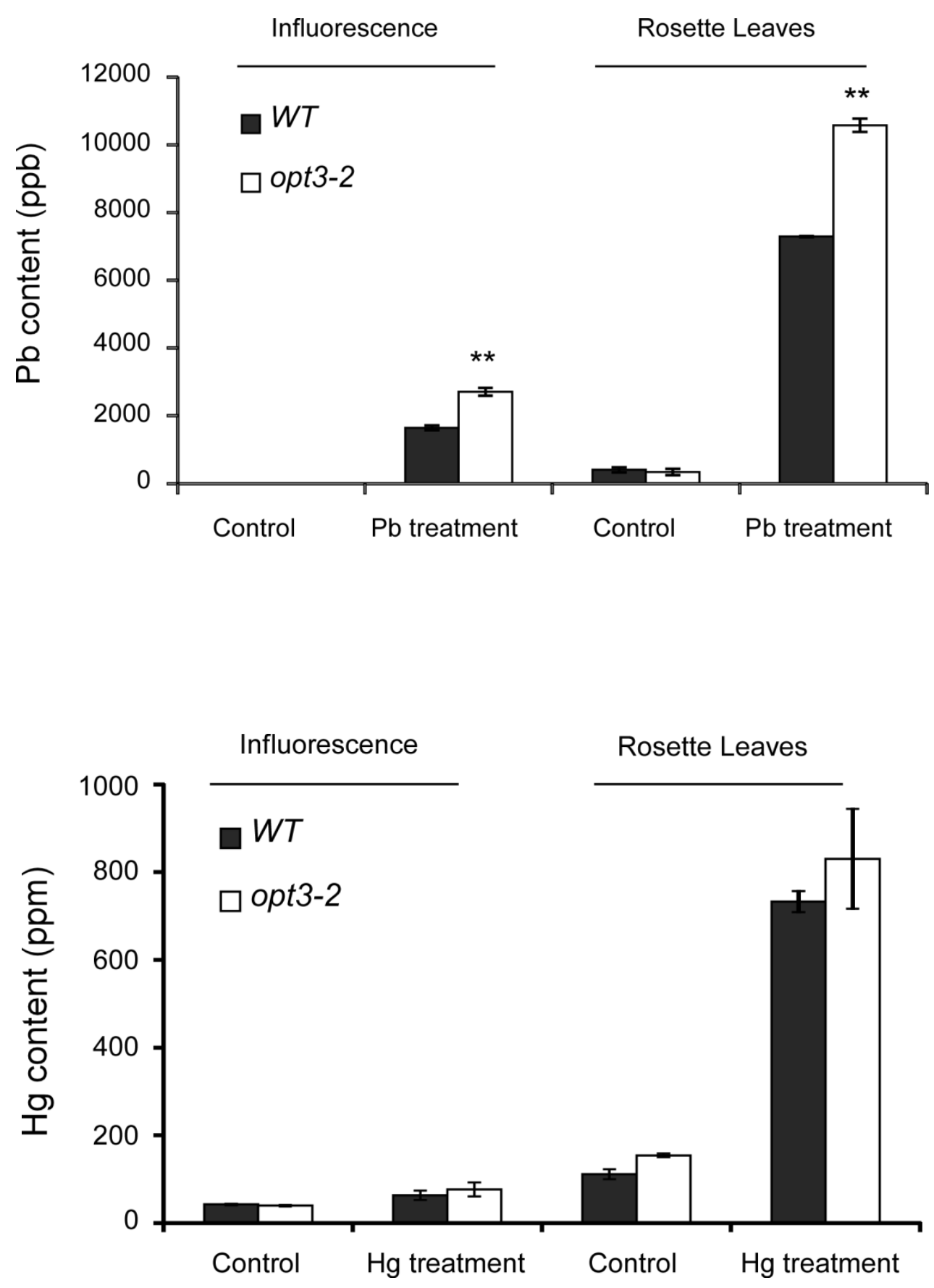

Figure 2-3. ICP-MS shows the amount of heavy metals in different above-ground tissues of both col-O and opt3-2 mutant. Left: $\mathrm{Pb}$ content; Right: $\mathrm{Hg}$ content. Plants used in this experiment are one month old. $n=2-3$, mean \pm SE. See Table A1-5 and A1-6 in appendix A for the tabular data. 


\section{Chapter 230}

a.

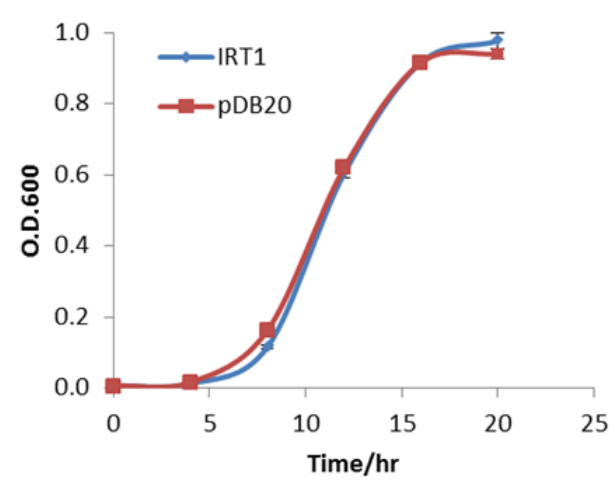

c.

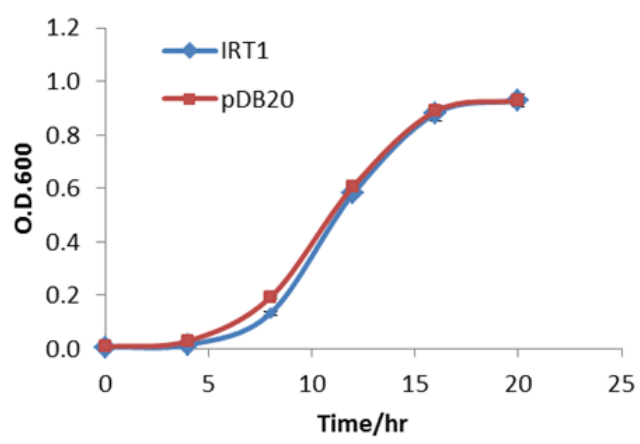

e.

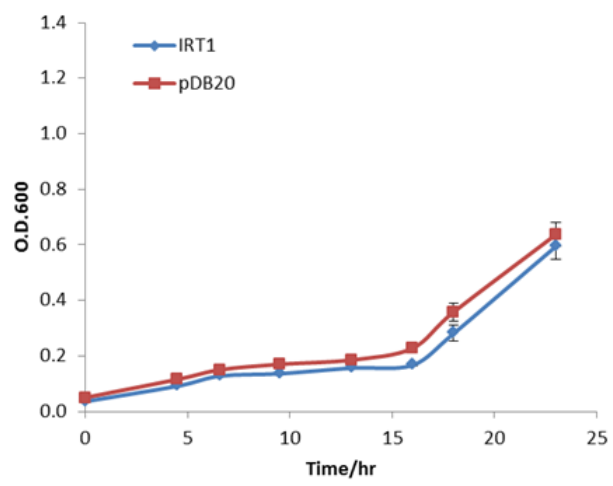

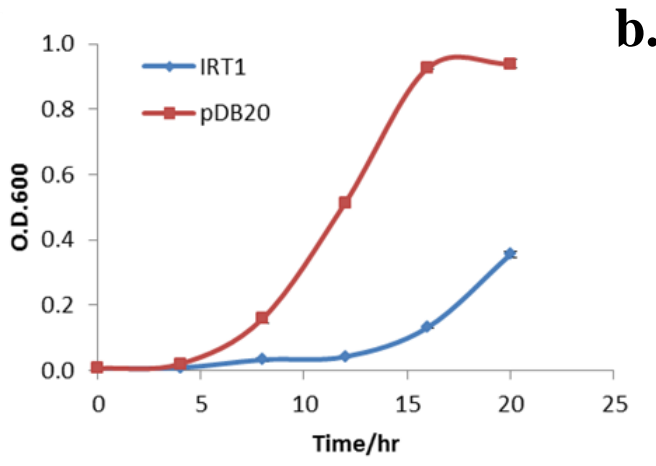

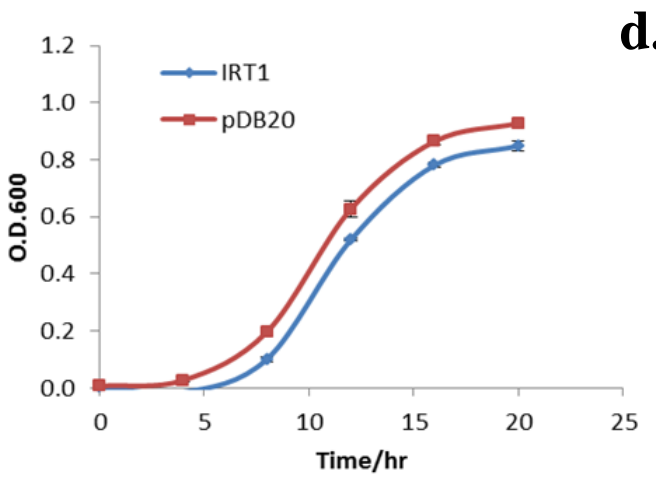

d.

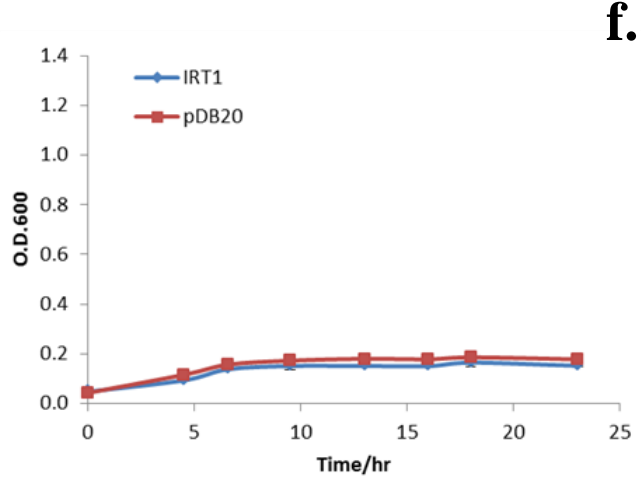

Figure 2-4. Comparison of different yeast transformants grown under various concentrations of heavy metal treatments. a) control, b) $5 \mu \mathrm{M} \mathrm{Cd}^{2+}$, c) $125 \mu \mathrm{M} \mathrm{Pb}^{2+}$, d) $250 \mu \mathrm{M} \mathrm{Pb}^{2+}$, e) $5 \mu \mathrm{M} \mathrm{Hg}^{2+}$ and f) $8 \mu \mathrm{M} \mathrm{Hg}^{2+}$. $\mathrm{n}=3$, mean $\pm \mathrm{SE}$ 


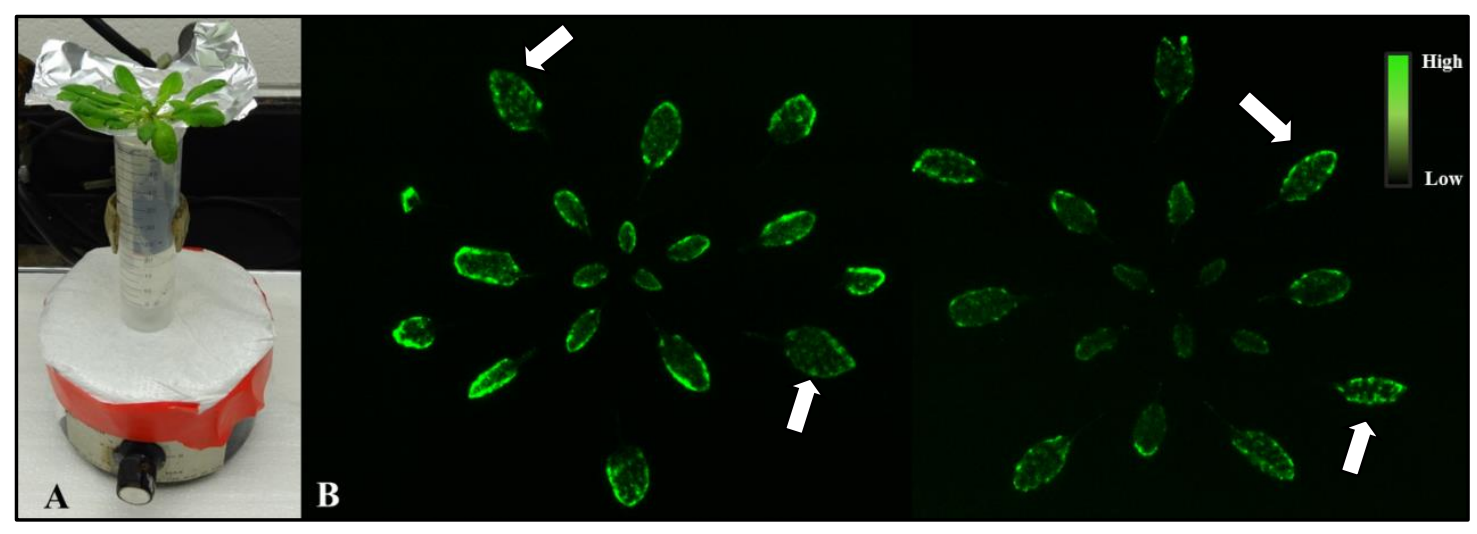

Figure 2-5. Phosphor image of ${ }^{203} \mathrm{~Pb}$ distribution in opt3-2 mutant and wild type plants. A, set-up for $\mathrm{Pb}-203$ uptake assay; B, Left one is wild type (col-O) and right one is opt3-2 mutant. Arrows are pointing out the leaves with concentrated spots which are very likely $\mathrm{Pb}$ accumulation in trichomes.

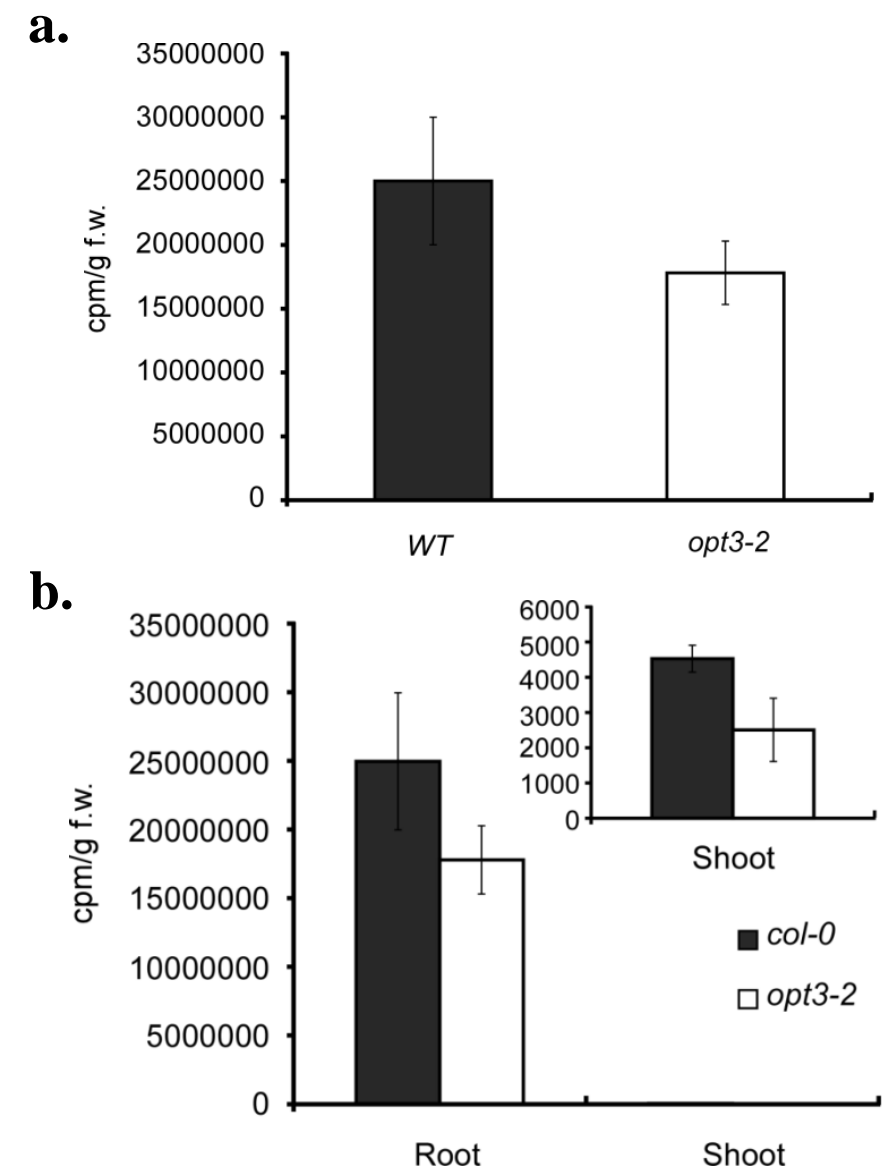

Figure 2-6. ${ }^{203} \mathrm{~Pb}$ distribution in opt3-2 mutant and wild type plants. a, total counts of $\mathrm{Pb}$ in the entire plant tissues (roots and shoots); $\mathbf{b}$, counts of $\mathrm{Pb}$ in roots and shoots separately. $n=4$, mean \pm SE. See Table A1-7 in AppendixA for the tabular data. 


\section{Chapter 232}

Table 2-1. Fractions of total ${ }^{203} \mathrm{~Pb}$ taken up in the plant transported into roots and shoots of the WT (col-0) and the opt3-2 mutant

\begin{tabular}{ccc}
\hline Type of plant & $\begin{array}{c}\text { \% of total }{ }^{\mathbf{2 0 3}} \mathbf{P b} \\
\text { Transported to the roots }\end{array}$ & $\begin{array}{c}\text { \% of total }{ }^{\mathbf{2 0 3}} \mathbf{P b} \\
\text { Transported to the shoots }\end{array}$ \\
\hline col-0 & $99.80 \pm 0.03$ & $0.20 \pm 0.03$ \\
\hline opt3-2 & $99.84 \pm 0.07$ & $0.16 \pm 0.07$ \\
\hline
\end{tabular}




\section{References}

1. Angelone, M., Bini, C., Trace element concentrations in soils and plants of Western Europe. In: Biogeochemistry of Trace Metals, 1992: p. 19-60.

2. Pilon-Smits, E., Pilon, M., Phytoremediation of Metals Using Transgenic Plants. Critical Reviews in Plant Sciences, 2002. 21(5): p. 439-456.

3. Marschner, H., Mineral Nutrition of Higher Plants Academic. Press, London, 1995.

4. Ensley, B.D., Rationale for use of phytoremediation. In: Phytoremediation of Toxic Metals - Using Plants to Clean up the Environment. Raskin, I. and Ensley, B.D., Eds., Wiley, New York, 2000: p. 1-12.

5. Salt, D.E., Blaylock, Michael, Kumar, Nanda P.B.A., Dushenkov, Viatcheslav, Ensley, Burt D., Chet, Ilan, Raskin, Ilya, Phytoremediation: A Novel Strategy for the Removal of Toxic Metals from the Environment Using Plants. Nat Biotech, 1995. 13(5): p. 468-475.

6. Hinchman, R.R., Negri, M. Cristina, Gatliff, Edward G., Phytoremediation: Using Green Plants to Clean up Contaminated Soil, Groundwater, and Wastewater. Argonne National Laboratory Hinchman, Applied Natural Sciences, Inc, 1998.

7. Clemens, S., Palmgren, M. G., Kramer, U., A long way ahead: understanding and engineering plant metal accumulation. Trends Plant Sci, 2002. 7(7): p. 309-315.

8. Ghosh, M., Singh, S. P., A review on phytoremediation of heavy metals and utilization of its byproducts. Asian Journal on Energy and Environment, 2005. 6(4): p. 214-231.

9. Krämer, U., Cadmium for all meals - plants with an unusual appetite. New Phytologist, 2000. 145(1): p. 1-3.

10. Lasat, M.M., Phytoextraction of toxic metals: a review of biological mechanisms. J Environ Qual, 2002. 31(1): p. 109-120.

11. Liu, D., et al., Uptake and accumulation of lead by roots, hypocotyls and shoots of Indian mustard [Brassica juncea (L.)]. Bioresource Technology, 2000. 71(3): p. 273-277.

12. Prasad, M.N.V., Metal hyperaccumulation in plants-biodiversity prospecting for phytoremediation technology. Electronic Journal of Biotechnology, 2003. 6(3): p. 285320. 
13. Xiao, S., Chye, M. L., Arabidopsis ACBP1 overexpressors are Pb(II)-tolerant and accumulate Pb(II). Plant Signal Behav, 2008. 3(9): p. 693-694.

14. Tangahu, B.V., et. al., A Review on Heavy Metals (As, Pb, and Hg) Uptake by Plants through Phytoremediation. International Journal of Chemical Engineering, 2011. 2011: p. 31 .

15. Lee, K.K., et. al., Cadmium and Lead Uptake Capacity of Energy Crops and Distribution of Metals within the Plant Structures. Journal of Civil Engineering, 2013. 17(1): p. 44-50.

16. Stacey, M.G., et al., The Arabidopsis AtOPT3 protein functions in metal homeostasis and movement of iron to developing seeds. Plant Physiol, 2008. 146(2): p. 589-601.

17. Garcia, M.J., et al., Shoot to root communication is necessary to control the expression of iron-acquisition genes in Strategy I plants. Planta, 2013. 237(1): p. 65-75.

18. Korshunova, Y.O., et al., The IRT1 protein from Arabidopsis thaliana is a metal transporter with a broad substrate range. Plant Mol Biol, 1999. 40(1): p. 37-44.

19. Vert, G., et al., IRT1, an Arabidopsis transporter essential for iron uptake from the soil and for plant growth. Plant Cell, 2002. 14(6): p. 1223-1233.

20. Porra, R.J., Thompson W.A., Kreidemann, P.E., Determination of Accurate Extinction Coefficients and Simultaneous Equations for Assaying Chlorophylls a and b Extracted with Four Different Solvents: Verification of the Concentration of Chlorophyll Standards by Atomic Absorption Spectroscopy. Biochimica et Biophysica Acta, 1989. 975: p. 348389.

21. Rogers, E.E., D.J. Eide, and M.L. Guerinot, Altered selectivity in an Arabidopsis metal transporter. Proc Natl Acad Sci U S A, 2000. 97(22): p. 12356-60.

22. Sharma, P., Dubey, R.S, Lead Toxicity in Plants. Braz. J. Plant Physiol., 2005. 17(1): p. $35-52$.

23. Morenoa, F.N., Andersonb, C.W.N, Stewartb, R.B., Robinson, B.H., Phytofiltration of mercury-contaminated water: Volatilisation and plant-accumulation aspects. Environmental and Experimental Botany, 2008. 62: p. 78-85.

24. Ferreira, C., Lopes Vieira, C., Azevedo, H., Caldeira, G., The effects of high levels of $\mathrm{Hg}$ on senescence, proline accumulation and stress enzymes activities of maize plants.

Agrochimica, 1998. 42(5): p. 209-218. 


\section{Chapter 235}

25. Spiller, S.C., Castelfranco, A. M., Castelfranco, P. A., Effects of Iron and Oxygen on Chlorophyll Biosynthesis: I. In Vivo Observations on Iron and Oxygen-Deficient Plants. Plant Physiology, 1982. 69(1): p. 107-111.

26. Pushnik, J.C., Miller, G.W., Iron regulation of chloroplast photosynthetic function: mediation of PSI development. J. Plant Nutr., 1989. 12: p. 407-421. 


\section{Chapter 3: Dynamics of Carbon Metabolism and Allocation in Arabidopsis Starch Mutants Using ${ }^{11} \mathrm{CO}_{2}$}

\subsection{Isotope Summary}

\begin{tabular}{|c|c|c|c|}
\hline Isotopes & Half-life & Decay Mode & $\mathbf{E}_{\max }$ \\
\hline${ }^{11} \mathrm{C}$ & \multirow{2}{*}{$20.3 \mathrm{~min}$} & $\beta^{+}(99.8 \%)$, & $0.96 \mathrm{MeV}\left(\beta^{+}\right)$, \\
& & $\mathrm{EC}(0.2 \%)$ & $0.511 \mathrm{MeV}$ annihilation photons $(199.6 \%)$ \\
\hline
\end{tabular}

\subsection{Background}

A fundamental understanding of how higher plants allocate and utilize their carbon-based resources for growth and development can be an important factor guiding future research toward attaining sustainability of both agricultural and bioenergy cropping systems.

Plants assimilate the fixed carbon into fructose and glucose during the day, which is then used for sucrose synthesis to support daily biological activities and plant growth, with the remaining sugars stored as transient starch that can be degraded at night back into soluble sugars to be used as a source of energy.

Sucrose is the primary sugar in plants and can be loaded into the phloem of source tissues (such as mature leaves) for long-distance transport to sink tissues (such as roots, young leaves and flowers). Other forms of sugars (such as fumarate, sorbitol, mannitol and raffinose) are used for long-distance transportation, but they are not preferred in Arabidopsis plants [1-3]. Fructose and glucose are building blocks for sucrose synthesis, 
as shown in Equation 1 below. This reaction is reversible. Sucrose can be degraded into fructose and glucose using enzymes, such as invertase or sucrose synthase.

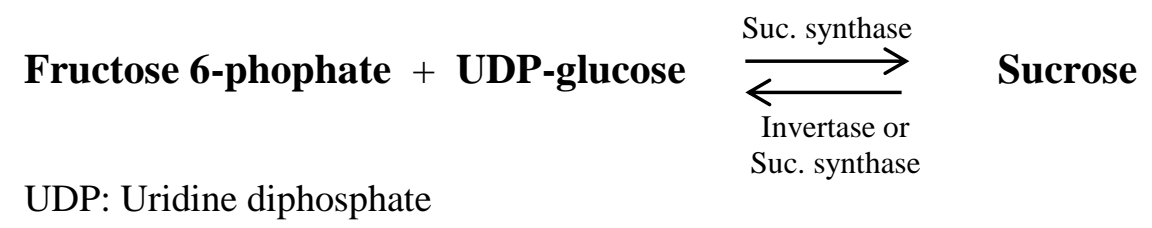

Equation 3-1: Sucrose synthesis and degradation.

Starch is the major storage carbohydrate in plants and there are two types of starch, amylose and amylopectin. Amylopectin, a highly branched glucan made of $\alpha-1,4-$ linked glucose units and branched at the $\alpha$-1,6-bonds, makes up $70 \%$ to $80 \%$ of the starch. The remaining $20 \%$ to $30 \%$ of the stored starch is in the form of amylose, a linear glucan containing few $\alpha$-1,6-branches. [4, 5] Plants assimilate and store a large portion of fixed carbon as transient starch in the chloroplast during the day, and degrade it into glucans at night to provide a steady supply of carbon as an energy source to support the normal growth of plants. [6-8] Photosynthetic partitioning of carbon into starch is well regulated. An imbalance in starch synthesis and degradation leads to changes in the pool size of key carbon metabolites, affecting the plant's overall growth and development. [4, 6, 9-14]

There are several important enzymes that play essential roles in starch synthesis and degradation (Fig. 3-1). Adenosine diphosphate (ADP)-glucose pyrophosphorylase (AGP) is considered the key enzyme for converting glucose-1-phosphate (glc-1P) into ADP-glucose (ADPG), which is then used for subsequent starch biosynthesis [15]. Plastidic phosphoglucomutase (PGM) is another important enzyme used in starch synthesis. PGM converts glucose 6-phosphate (glc-6P) to glc-1P, which is a precursor of 
ADPG (Equation 2) [16] and is found in both the plastids and the cytosol [17]. Starch excess1 protein (SEX1), reported by $\mathrm{Yu}[18]$, is a protein with significant homology to the starch granule-bound protein (R1) found in potato and plays an important role in regulating starch mobilization by controlling the phosphate content of starch.

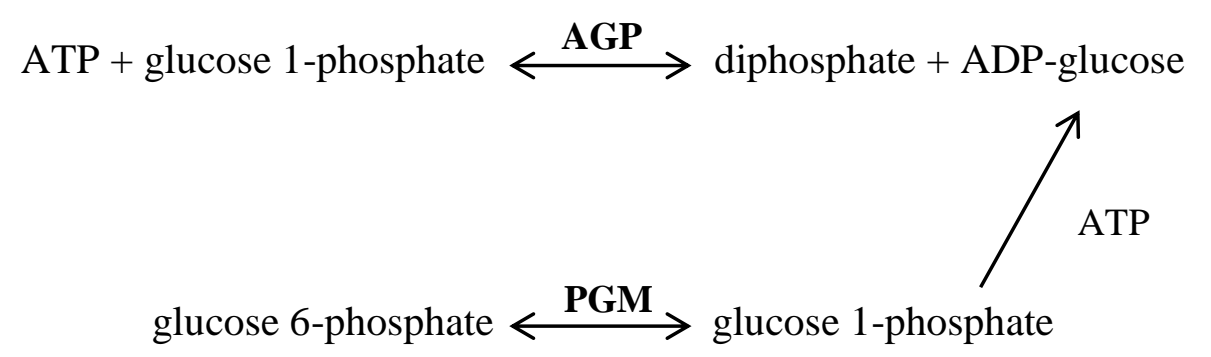

Equation 3-2: Biological functions of the enzyme ADP-glucose pyrophosphorylase (AGP) and phosphoglucomutase (PGM).

Starch metabolism has been extensively studied in the past [4-6, 8, 19], leading to sound knowledge of the pathways and the enzymes involved. However, there are still many open questions regarding the signals and mechanisms regulating starch metabolism. To further explore this topic, several Arabidopsis starch mutants were used to target specific steps in the starch metabolism pathway as well as to see how plants respond physiologically to diurnal (time-of-day) effects.

\section{Starch excess mutants}

Starch excess Arabidopsis mutants exhibit over-accumulation of starch in leaf tissues due to an impairment of starch degradation at night. The sexl and the sex4 mutants have been studied in the past $[9,18,20]$. The sexl mutant was shown, in Trethewey and Rees' study [21], to be incapable of exporting glucose produced by starch hydrolysis in the chloroplast. However, in Yu's study years later [18], the sexl mutant 
was shown to lack the SEX1 protein instead of a hexose transporter. The sex4 mutant is deficient in a chloroplastic endoamylase, which reduces the rate of starch degradation [20]. Both starch excess mutants were found to contain a large amount of starch content in the early morning compared to the wild type Arabidopsis (Columbia-0), and this high starch level phenotype arises from the gradual accretion of starch during development. Also, an altered carbon partitioning to soluble sugars was observed in the sex 4 mutant [9].

\section{Starch deficient mutants}

In general, starch deficient mutants exhibit little to no starch synthesized in tissues. The $a d g l$ and the $p g m-1$ mutants have been well studied in the past. The $a d g l-1$ mutant is a monogenic recessive mutation with less than $2 \%$ of the starch of the wild type. Bahaji's research [15] showed that the adg1-1 mutant has much less ADGase activity in leaf extracts, $2 \%$ of the ADGase activity of the wild type, but has comparable amounts of ADP-glucose. Thus, there might be an alternate pathway to synthesize ADPglucose in the adgl-1 mutant and subsequently to synthesize starch. The pgm-1 mutant lacks the plastidic PGM enzyme activity compared to the wild type and has very low levels of starch synthesis. Since the assimilated carbon cannot be used to synthesize starch, a large amount of the carbohydrates are stored in the form of soluble sugars. Research has shown that the pgm-1 mutant has an increased pool size of sucrose and hexose sugars $[16,22]$.

The plant carbohydrate metabolism is not only dependent on the internal carbon status, but also can be affected by hormone levels, such as the indole-3-acetic acid 
(IAA/Auxin) level in plants. [5, 23] IAA is a plant hormone that plays a role in many diverse developmental and cellular processes impacting axis formation and patterning $[24,25]$, especially in regulating root development [26]. Most particularly, this hormone is known to cause extensive lateral root patterning as well as extensive root hair formation at the expense of root elongation. A recent maize study [23] showed that by using the ${ }^{11} \mathrm{CO}_{2}$ labeling technique, in combination with a continuous root treatment with IAA, an alteration of new carbon partitioning into soluble sugars was affected along with starch content. For example, an increasing amount of ${ }^{11} \mathrm{C}$-partitioning into sucrose rather than glucose and fructose sugars was observed in the IAA treated plants.

In this study, we leverage a combination of molecular tools $\left({ }^{12} \mathrm{C}\right.$ and ${ }^{11} \mathrm{C}$ analyses $)$ through the use of starch mutants (sex1-1, adg1-1, pgm-1) and the plant's natural evolutionary trait of diurnal cycling of carbon resources to explore the effects of these parameters on the terminal phenotype.

Many studies have been done to investigate these starch mutants individually, but a detailed comparison has not yet been conducted. In addition, previous carbon metabolism studies have focused on ${ }^{12} \mathrm{C}$ analysis, which demonstrates the total amount of carbon in different forms in the plant tissues, but is limited in its use for detecting carbon flux in a short time window. Carbon-14 $\left({ }^{14} \mathrm{C}\right)$ chase assays have been widely used to track the dynamics of carbon partitioning, however, ${ }^{14} \mathrm{C}$ is a weak $\beta^{-}$emitter with a long halflife requiring a long exposure time to acquire the amount of signal needed for analysis. In our study, ${ }^{11} \mathrm{CO}_{2}$ was used to explore the dynamics of carbon metabolic partitioning and allocation during the daytime. Carbon-11 $\left({ }^{11} \mathrm{C}\right)$ decays by positron emission with a short half-life of $20.36 \mathrm{~min}$. The positrons can be detected by a phosphor screen with high 
sensitivity and it gives excellent signal resolution with a relatively short exposure time,

even though the concentration of ${ }^{11} \mathrm{C}$ is very low in plant tissues. Thus, ${ }^{11} \mathrm{C}$ analysis is a more efficient technique to study the quickly changing concentration of carbon in plant tissues. All of the ${ }^{11} \mathrm{C}$ studies were conducted at Brookhaven National Laboratory (BNL), which has a unique facility capable of producing and administering discrete doses of radioactive ${ }^{11} \mathrm{CO}_{2}$ to intact plants.

\subsection{Materials and Methods}

\subsubsection{Materials}

Bleach used for seed sterilization was purchased from Pure Bright (Columbia, MO). All other chemicals used in this study were purchased from Sigma Aldrich (St. Louis, MO).

\subsubsection{Plant Growth Conditions}

Seeds used in this study were purchased from The Arabidopsis Information Resource (TAIR).

Seeds were surface-sterilized with a $35 \%$ bleach solution containing $0.1 \%$ Triton $\mathrm{X}-100$ for $15 \mathrm{~min}$, and rinsed several times with sterile water. Prior to germination seeds were placed in the cold $\left(4^{\circ} \mathrm{C}\right)$ for $2-3$ days to align seedling germination times. For routine growth, seedlings were grown in an agar medium containing fullstrength Hoagland's salts, 0.05\% morpholinoethanesulfonic acid (MES) and 5\% agar. The $\mathrm{pH}$ of the medium was adjusted to 6.0 with $\mathrm{NaOH}$ solution. Seedlings were 
grown at $22^{\circ} \mathrm{C}$ under fluorescent white light $\left(100 \mu\right.$ mole $\left.\mathrm{m}^{-2} \mathrm{~s}^{-1}\right)$ with a $12 \mathrm{~h}$ (day)/12 $\mathrm{h}$ (dark) photoperiod.

\subsubsection{Starch Staining Assay}

Either 48-well or 96-well plates were used for this assay. Two-week old seedlings were immersed in methanol and kept at $80^{\circ} \mathrm{C}$ for $10 \mathrm{~min}$ before decanting all of the extraction solution. This step was repeated another time to ensure complete chlorophyll removal from the leaves. An aliquot of iodine solution (containing $0.34 \% \mathrm{I}_{2}$ and $0.68 \%$ KI) was then added to each well of the plate to cover the whole seedling. The seedlings were stained for 30 min before being rinsed with deionized water. An upright microscope (Olympus Vanox AHBT3) with a color digital camera (provided by the MU Molecular Cytology Research Core Facility) was used for taking images. Leaf starch staining was done both in the early morning ( 1 hour after the light turned on) and late afternoon ( 7 hours after the morning assay). Root tips were cut off from the root just before imaging them.

\subsection{4 ${ }^{11} \mathrm{CO}_{2}$ Production, Pulsing and Incubation Apparati}

${ }^{11} \mathrm{CO}_{2}$ was produced via the ${ }^{14} \mathrm{~N}(\mathrm{p}, \alpha){ }^{11} \mathrm{C}$ nuclear transformation [27] from a 20 $\mathrm{mL}$ target filled with high-purity nitrogen gas (400 mL @ STP) using $18 \mathrm{MeV}$ protons from the TR-19 (Ebco Industries Ltd, Richmond, BC, Canada) cyclotron at BNL, and captured on molecular sieves $(4 \AA)$. The captured ${ }^{11} \mathrm{CO}_{2}$ was desorbed and quickly released into an air stream at $200 \mathrm{~mL} / \mathrm{min}$ as a discrete pulse for labeling the seedlings in customized sealed incubation chambers (Fig. 3-2). Seedlings within the chamber were 
pulse-fed ${ }^{11} \mathrm{CO}_{2}$ for 1 minute, and then chased with normal air for the duration of the exposure.

Two types of customized apparati were used as sealed systems during ${ }^{11} \mathrm{CO}_{2}$ pulsing and incubation of the seedlings. For sugar analysis, a square petri dish (without its cover) with seedlings grown in agar media was kept in a sealed tip box (Fig. 3-2A). For the carbon allocation assay, $1.5 \mathrm{ml}$ Eppendorf ${ }^{\mathrm{TM}}$ tubes with seedlings grown in agar media were placed in a sealed customized tube rack (Fig. 3-2B). Both apparati have two connections, one for ${ }^{11} \mathrm{CO}_{2}$ introduction and one to serve as an exhaust for the closed system. Fixed red/blue LED lights were placed on top of the tip box/ tube rack to ensure normal photosynthesis at $100 \mu$ mole $\mathrm{m}^{-2} \mathrm{sec}^{-1}$. Light intensity was monitored before each experiment using a LiCor Quantum sensor.

The ${ }^{11} \mathrm{CO}_{2}$ experiments were conducted both in the early morning (1 hour into the start of the photoperiod) and late afternoon (7 hours after the AM experiment) as described in the Starch Staining Assay.

\subsubsection{Tissue Extraction and Soluble Sugars Analysis}

Seedlings for sugar analysis (wild type and three starch mutants) were grown on a square plate filled with half-strength MS agar medium for 2-3 weeks. After ${ }^{11} \mathrm{CO}_{2}$ pulsing and incubation for $20 \mathrm{~min}$, all the leaf tissues were harvested and immediately frozen in liquid nitrogen and ground by hand in a pre-weighed and pre-chilled Eppendorf ${ }^{\mathrm{TM}}$ tube. Methanol, four-times the milligram mass of the powder, was added to the centrifuge tube, vortexed (VWR analog vortex mixer; Sigma-Aldrich Corp., St. Louis, MO, USA) and then sonicated (Branson Bransonic 32; Sigma-Aldrich Corp. St. Louis, MO, USA) at $0{ }^{\circ} \mathrm{C}$ 
for $10 \mathrm{~min}$. Additional vortexing was performed during this period to ensure complete mixing. Afterward the Eppendorf tubes were centrifuged for $2 \mathrm{~min}$ at 15,000 rpm to separate the insoluble portion from the soluble one. The insoluble portion contained mostly cell-wall polymers and starch. The soluble portion contained small soluble compounds, including soluble sugars. These sugars were separated and analyzed by thin layer chromatography (TLC) [28, 29]. Glass backed $\mathrm{NH}_{2}$-silica HPTLC-plates $(200 \mu \mathrm{m}$, w/UV254) were used for the sugar separation (Sorbent Technologies, Atlanta, GA, USA). Plates were pre-spotted with $2 \mu \mathrm{L}$ of the sugar standards (including 4, 3, 2, 1, 0.5 and $0.2 \mathrm{mM}$ of glucose, sucrose and fructose) using a semi-automatic Linomat 5 sample applicator (Camag Scientific Inc., Wilmington, NC, USA) for a high precision of spot size and sample volume. Aliquots $(2 \mu \mathrm{l})$ of leaf extract were also spotted. TLC plates were developed using a mobile phase consisting of 75:25 acetonitrile/water (v/v). The developed TLC plates were imaged using a Phosphor screen (FujiFilm BAS-MS 2040, GE Healthcare Biosciences, PA) to determine the yield of each radiolabeled sugar. The plates were then heat-treated $\left(200^{\circ} \mathrm{C}, 10 \mathrm{~min}\right)$ to initiate a chemical reaction of individual sugars with the amino functionalized Si-support giving a fluorescence marker detectable under long wavelength (365 nm) UV light [28]. Digital photographs were taken of the fluorescent markers to provide a measure of the ${ }^{12} \mathrm{C}$-sugar yields. Image Quant TL software was used to analyze both the radiographic and the digital images to determine the amount of carbon-11 $\left({ }^{11} \mathrm{C}\right)$ or carbon-12 $\left({ }^{12} \mathrm{C}\right)$ sugars. Linear Log regression was used for plotting standard curves generated from the ${ }^{12} \mathrm{C}$-sugar standards. 


\section{Chapter $3 \quad 45$}

\subsubsection{Carbon Allocation}

Seedlings for carbon allocation analysis were grown for 2-3 weeks in $1.5 \mathrm{ml}$ Eppendorf ${ }^{\mathrm{TM}}$ tubes filled with agar media. After ${ }^{11} \mathrm{CO}_{2}$ pulsing and 1 hour incubation, the entire seedlings were removed from the agar media and cut into roots and shoots, then stored in respective vials filled with methanol. Tissues were later counted in a $\mathrm{NaI}(\mathrm{Tl})$ well-counter. The remaining tubes, containing agar medium, were also placed individually into empty vials for counting, which indicated the amount of ${ }^{11} \mathrm{C}$-labeled root exudates. This experiment was conducted both in the early morning and late afternoon as described in the ${ }^{11} \mathrm{CO}_{2}$ production, pulsing and incubation apparatus.

The percentage of ${ }^{11} \mathrm{C}$-phytosynthates transported to below-ground and exuded from the roots was calculated as follows:

$$
\begin{aligned}
& \% \text { of }{ }^{11} \mathrm{C} \text {-phytosynthates below }=\frac{\mathrm{A}_{\text {roots }}+\mathrm{A}_{\text {exudates }}}{\mathrm{A}_{\text {shoots }}+\mathrm{A}_{\text {roots }}+\mathrm{A}_{\text {exudates }}} \times 100 \% \\
& \% \text { of }{ }^{11} \mathrm{C} \text {-phytosynthates exudates }=\frac{A_{\text {exudates }}}{A_{\text {shoots }}+A_{\text {roots }}+A_{\text {exudates }}} \times 100 \%
\end{aligned}
$$

$\mathrm{A}_{\text {shoots }}, \mathrm{A}_{\text {roots }}$ and $\mathrm{A}_{\text {exudates }}$ indicate the activities measured in the shoots, root tissues and in the gel (root exudates). 


\section{Chapter 3}

\subsubsection{Phosphor Imaging}

After the Carbon Allocation assay, some of the seedlings were removed from the $1.5 \mathrm{ml}$ Eppendorf $^{\mathrm{TM}}$ tubes and exposed to a phosphor imaging screen (FujiFilm, BAS-MS grade) for 20 seconds, which was then analyzed using a phosphor-imager (Typhoon ${ }^{\mathrm{TM}}$ FLA 9000, GE Healthcare).

\subsubsection{Root Morphology}

Seedlings were prepared using the same method as described in the Tissue Extraction and Soluble Sugar Analysis. The area of the entire roots and lateral roots was measured by RootSnap software (CID Bio-Science, Inc.).

\subsubsection{Root Elongation and Gravitropic Response}

Seedlings were germinated vertically in full-strength Hoagland's agar medium for

5 days before transferring into fresh agar medium under the same conditions. Seedlings were placed perpendicular to the ground for a day as time zero. Then the plates containing the seedlings were rotated $90^{\circ}$. Pictures were taken over the first 24 hours. Root lengths and angles were measured later by ImageJ software.

\subsubsection{Statistical Analysis}

Data was subjected to the Student t-test for unpaired samples assuming an unequal variance. Statistical significance levels were assigned to the following rating scale $(*, \mathrm{p}<0.05 ; * *, \mathrm{p}<0.01 ; * * *, \mathrm{p}<0.001)$ 


\subsection{Results}

\subsubsection{Plant Growth}

Compared to the wild type (WT, col-0), both starch excess and starch deficient mutants showed stunted root growth (Fig. 3-3). Their roots grew slower than WT roots (Fig. 3-4). Among the starch mutants, adgl-1 and pgm-1 mutants have significantly more total root area developed than the sex $1-1$ and the WT plants, especially lateral root development (Fig. 3-3 and Fig. 3-5). The chlorophyll levels in the starch mutants were similar to the WT. However, among the starch deficient mutants, the pgm-1 mutant had around $15 \%$ more chlorophyll than the adgl-1 mutant, although both mutants were deficient in starch.

\subsubsection{Plant Physiology}

\section{Starch content}

The starch staining analysis on leaf showed that the WT had more starch present in the leaves in afternoons compared to mornings. However, under either time point, the WT did not contain as high a level of starch as the sex $1-1$ mutant did. The sex $1-1$ mutant leaves contained more starch than all the other plant genotypes in both mornings and afternoons (Fig. 3-6), but it showed decreased staining areas on the leaf in the afternoon, which indicated that some starch was degraded into other metabolites. There was little to no starch in the adgl-1 and the pgm-1 mutant leaves regardless of the time of the day. The root tips were also stained for starch, which showed that both WT and sex1-1 mutants contained visible amounts of starch granules. However, in contrast to leaves (Fig. 3-6), the sex 1-1 mutant did not show significantly more starch in the root tip than 
the WT (Fig. 3-7). At the same time, there was a lack of starch in the root tips of the adgl-1 and the pgm-1 mutants.

\section{Carbon allocation}

Results from the ${ }^{11} \mathrm{CO}_{2}$ experiments showed that the starch mutants have altered carbon relocation. The WT tended to transport more assimilated carbohydrates to the belowground segments (roots + root exudates) in the afternoon than in the morning, while both the adg1-1 and the pgm-1 mutants consistently showed $36 \%$ more of assimilated carbon being transported into the belowground segments than the WT did in both mornings and afternoons. The sex 1-1 mutant transported 30\% more assimilated carbon to the belowground segments than the WT did in the morning but $17 \%$ less than the WT in the afternoon.

The root exudation data indicated that $2 \%$ or lower of assimilated carbon was secreted to the rhizosphere through plant roots and the amount of root exudation in all types of Arabidopsis seedlings in this study exhibited a reduction in afternoons (Fig. 3-8). Around $1 \%$ of the assimilated carbon in the WT was exuded to the rhizosphere. The sexl1 mutant had a greater percentage, around $1.6 \%$ of assimilated carbon, being secreted from the roots in the mornings, but only $0.8 \%$ in the afternoons. Among the starch deficient mutants, the adg $1-1$ had a significantly higher amount, around $2 \%$, of the assimilated carbon exuded into the rhizosphere in the mornings (even higher than the WT), and decreased to the WT level in the afternoons; whereas the pgm-1 mutant exhibited the same levels of root exudates as the WT in both mornings and afternoons. 


\subsubsection{Carbon Metabolism}

The combination of ${ }^{12} \mathrm{C}$ and ${ }^{11} \mathrm{C}$ data showed that the starch mutants have differing patterns of carbon partitioning from the WT.

\section{Soluble sugars}

Based on the ${ }^{12} \mathrm{C}$ AM data (Fig. 3-9a), the sex $1-1$ mutant contained similar amounts of all three soluble sugars as did the WT. The adgl-1 and the pgm- 1 mutants showed significantly more fructose and glucose present in the leaves than the WT, and showed a comparable amount of sucrose to the WT. In the ${ }^{12} \mathrm{C}$ PM data (Fig. 3-9b), the adgl-1 and the pgm-1 mutants also showed higher amounts of fructose and glucose than the WT as well as an increased amount of sucrose in the leaves compared to the WT.

Based on the ${ }^{11} \mathrm{C}$ AM data (Fig. 3-9c), all types of Arabidopsis seedlings showed a large fraction of fixed ${ }^{11} \mathrm{C}$ assimilated into sucrose synthesis and relatively less into fructose and glucose synthesis. The sex $1-1$ mutant had a trend of more ${ }^{11} \mathrm{C}$ labeled soluble sugars partitioned in the leaves than did the WT, but it was not statistically significant. The $\operatorname{adg} 1-1$ and $\mathrm{pgm}-1$ assimilated significantly more ${ }^{11} \mathrm{C}$ into all three soluble sugars, except that $p g m-1$ did not show as much ${ }^{11} \mathrm{C}$-sucrose as the adg $1-1$ mutant did (16\% vs. $21 \%)$. The ${ }^{11} \mathrm{C}$ PM data showed a similar trend as the AM data with some differences. Compared to the ${ }^{11} \mathrm{C}$ AM data, all types of Arabidopsis in PM (Fig. 3-9d) tended to have less ${ }^{11} \mathrm{C}$ partitioned into ${ }^{11} \mathrm{C}$-fructose and ${ }^{11} \mathrm{C}$-glucose. As to sucrose, the sex $1-1$ mutant showed decreased (from $17 \%$ to $10 \%$ ), while the pgm-1 mutant showed increased (from $16 \%$ to $21 \%$ ) ${ }^{11} \mathrm{C}$ partitioned into ${ }^{11} \mathrm{C}$-sucrose in PM than in AM. The 
WT and the adgl-1 did not show any notable changes in the amount of ${ }^{11} \mathrm{C}$ partitioned into sucrose from AM to PM.

Insoluble carbohydrates

The three starch mutants also showed differing patterns in carbon partitioning to insoluble carbohydrates (Fig. 3-10). Around half of the fixed ${ }^{11} \mathrm{C}$ was partitioned into insoluble carbohydrates in the WT and it did not change from AM to PM. Compared to the WT, all three starch mutants had a lower fraction of fixed ${ }^{11} \mathrm{C}$ partitioned into insoluble carbohydrates in the early mornings. This trend was also observed in the afternoons.

\subsubsection{Root Gravitropic Response}

The sex1-1 mutant showed a root gravitropic response similar to the WT (Fig. 34). However, both of the starch deficient mutants exhibited significantly slower root tip turning rates than the WT within the first 6 hours of the experiment, but a similar turning rate as the WT at later times. The root turning rates did not stay constant. All Arabidopsis seedlings showed two major increases in root turning rates during the experiment regardless of the genotype; one was within the first three hours and the other was between 6 to 9 hours into the experiment.

Root elongation rates were also measured along with the root gravitropic response. Both the starch excess and the starch deficient mutants exhibited slower root elongation rates than the WT. The root elongation rates fluctuated as did the root turning rates. However, they were not in sync, but staggered with the increases occurring in root turning rates. 


\subsection{Discussion}

\subsubsection{Carbon Metabolism and Allocation}

Our results show that the starch excess mutant and the starch deficient mutants have notably altered patterns of carbon partitioning and carbon allocation compared to the wild type. According to the conceptual model mentioned in Agtuca's study [23], the

${ }^{11} \mathrm{C}$ data demonstrates snapshots of the dynamics of carbon flux, and is combined with ${ }^{12} \mathrm{C}$ analysis, which demonstrates the carbon pool size (such as the sugar pool size), to gives a full picture of the fluctuation of carbon metabolism in both the starch mutants and the WT.

\section{$\underline{\text { Wild Type Arabidopsis }}$}

The combination of ${ }^{12} \mathrm{C}$ and ${ }^{11} \mathrm{C}$ data for the WT leaf tissue showed continuous carbon influx (as soluble sugars) to the soluble sugar pools (Fig. 3-9c and d) during the day but no change in the sugar pool sizes (Fig. 3-9a and b), suggesting that the assimilated soluble sugars (such as fructose, glucose and sucrose) were used in the downstream carbon metabolic pathways or translocated to other sink tissues in order to keep the size of the sugar pools constant. Our insoluble carbohydrate data (Fig.3-10), root allocation and exudation data (Fig.3-8) are consistent with the hypothesis.

A diurnal effect on carbon partitioning in the WT was observed. Less ${ }^{11} \mathrm{C}$ fructose and ${ }^{11} \mathrm{C}$-glucose was found in the leaves of the WT in the afternoon (Fig. 3-9d), which suggests that the carbon portioning was likely re-directed. Fructose, glucose and sucrose are under constant inter-conversions as shown in Equation 1, and the reaction might be shifted towards sucrose in the afternoon, which means fructose and glucose 
were removed from their sugar pools. However, no increase was observed in the downstream sugar metabolism, such as sucrose and starch synthesis in the WT (Fig. 3-9 and 3-10). The carbon allocation data (Fig. 3-8a) showed an increase in translocation of carbohydrates to belowground, which suggests that more fructose and glucose were used to synthesize sucrose in the afternoon, which was then transported to the roots of the WT.

The root allocation and exudation data showed that most of the carbohydrates translocated to belowground were retained in the roots rather than secreted into the rhizosphere. There was also a diurnal effect observed for carbon allocation to belowground. More carbohydrates were translocated to belowground in the afternoon, with more retained in the roots and a similar level in the root exudates compared to the morning data.

$\underline{\text { sex } 1-1 \text { Mutant }}$

Previous research has shown that the sexl (TC26) mutant exhibits a normal rate of starch synthesis similar to the WT during the day, but a slower rate of starch degradation at night $[9,30]$. Over time the sex 1 mutant tended to accumulate much more starch in the leaves (Fig. 3-7). Our ${ }^{11} \mathrm{C}$ data showed that the leaves of the sex $1-1$ mutant exhibited a similar synthetic rate of the insoluble carbohydrates (mainly starch) as the WT (Fig. 3-10). This impairment of starch degradation could lead to an imbalance between starch synthesis and degradation, which may subsequently affect carbon partitioning in the mutant. Zeeman's study [9] showed that the sex4 mutant has elevated amounts of fructose and glucose during the day, whereas the amount of sucrose was comparable to the WT. However, prior to our study, there was no detailed sugar analysis 
of the sexl-1 mutant. Our data shows that the sugar pools in the sexl-1 mutant leaves were not disturbed and exhibited similar levels of soluble sugars as the WT (Fig. 3-9a and b). The amount of fixed ${ }^{11} \mathrm{C}$ in leaves, however, tended to be partitioned more into the fructose and glucose pools compared to the WT. The increased carbon influx during the daytime did not change either the size of the soluble sugar pools or the amount of assimilated insoluble carbohydrates, which suggests that the soluble sugars were likely translocated rapidly, primarily as sucrose, from the source leaves to the other sink tissues, such as the roots in this case. Compared to the WT, a higher amount of carbohydrates in the belowground of the sex 1-1 mutant was observed in the early morning (Fig 3-8), but was significantly reduced in the afternoon, to levels below that of the WT. The mechanism of the reduced amount of belowground carbohydrates in the sex $1-1$ mutant is still unknown. A reduction of root exudates in the sex $1-1$ mutant in the afternoon was also observed (Fig. 3-8b).

\section{$\underline{\text { adg } 1-1 \text { and pgm-1 Mutants }}$}

The adg 1-1 and pgm-1 mutants are deficient in starch synthesis and only produce around $2 \%$ of the starch that the wild type does $[15,16,31,32]$. In our ${ }^{11} \mathrm{C}$ study, a low percentage of assimilated insoluble carbohydrates in the starch deficient mutants was also observed (Fig. 3-10). Previous research has shown that the carbon in the pgm mutant was redirected into soluble sugar synthesis, such as fructose, glucose and sucrose synthesis [16]. In our study, the same trend was seen in both the adgl-1 and the pgm-1 mutants (Fig. 3-9). In addition, we found that neither starch deficient mutant had elevated sucrose in the early morning compared to the WT, but both showed an increase to twice the quantity of the WT in the afternoon (Fig. 3-9a and 3-9b). Casper has shown that the 
soluble sugars in the pgm mutant were consumed rapidly at night [16] since there is little starch available that can be used to re-generate sugars. The sugars are then regenerated rapidly through photosynthesis in the early morning, refilling the sugar pools. Thus, it is likely that the starch deficient mutants were completely depleted in sucrose overnight and just started to synthesize and refill the sucrose pool, elevating the amount of sucrose to the level observed in the WT at the time of measurement (Fig. 3-9c). In addition, it is possible that large amounts of sucrose were exported into the roots at the same time (Fig 3-8a), which may have contributed to the relatively low sucrose levels observed in both mutants in the early morning.

\subsubsection{Root Morphology and Gravitropic Response}

An imbalance in starch regulation not only alters carbon partitioning and allocation, but also subsequently affects root morphology and root gravitropic responses. We found that the starch deficient mutants tended to have a greater number of lateral roots and total root area than the wild type (Fig. 3-3 and 3-5). This phenotype might be the result of the increased carbohydrate translocation to the belowground segments observed in these starch deficient mutants (Fig. 3-8). The carbohydrates translocated to the belowground segments could be the excess soluble sugars produced by photosynthesis, or other carbon compounds serving as signaling molecules, such as auxin, or both of them. Auxin has been shown to regulate lateral root growth. Thus, it is possible that the auxin level in these starch deficient mutants is altered [33] and is responsible for the increased lateral root growth, which creates a large sink for carbohydrates. However, the mechanism of this elevated carbohydrate translocation to the roots in these starch 
deficient mutants is still unknown. This is worth studying in the future with the aim of developing methods to improve root growth and increase root biomass.

The root gravitropic response has been shown to be related to the starch granules in plant root tips. A lack of starch in plants can result in a loss of such response [33-35]. For example, the results of our root gravitropic response experiments showed that the starch deficient mutants had reduced root turning angles (Fig. 3-4). The phosphor images of the starch deficient mutants (Fig. 3-5) also showed "zig-zag" root growth patterns. However, an over-accumulation of starch in plants, such as in the sexl-1 mutant, did not show any impaired root gravitropic response. Our hypothesis is that starch is an initial physical stimulus to root columbella cells in responding to the gravity and is not the only factor that causes the gravitropic response. Our root gravitropic experiments showed that the starch deficient mutants can eventually turn toward the ground even though they did not show any starch granules in the root tips (only an observation). Followed by the starch stimulus, a chemical signal was then induced to one side of the root cap cells to expand. For example, the Cholodny-Went theory indicates that auxin plays a key role in the root gravitropic response [36-38]. Such a mechanism explains: 1) why there was only a significant difference between the starch deficient mutants and the WT at the beginning of the gravitropic response, but not in the later stages (Fig. 3-4); and 2) why it showed a staggered pattern between root turning rate and root growth rate in our root gravitropic response experiments (Fig. 3-4). 


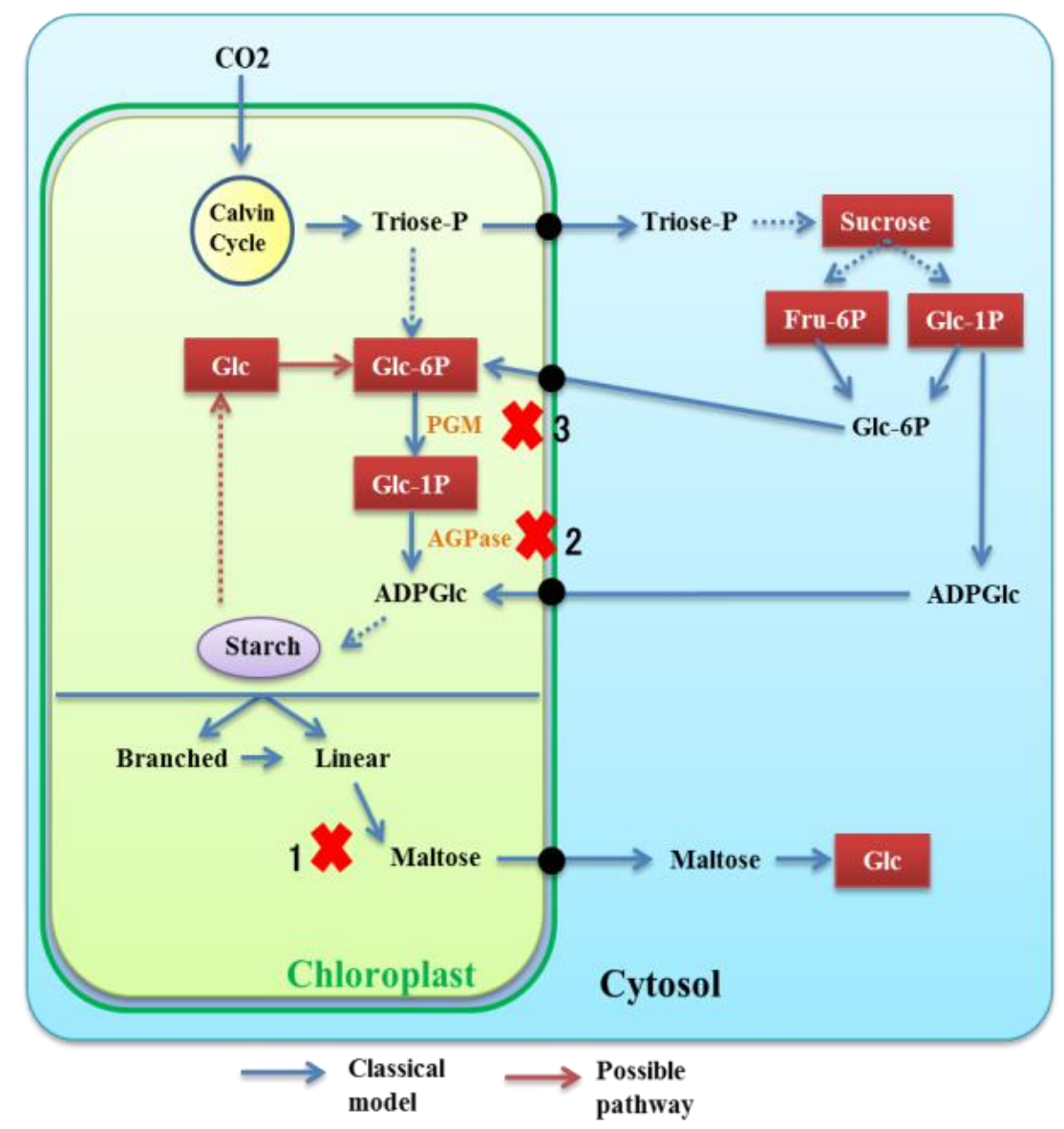

Figure 3-1. Brief summary of carbon fixation and downstream carbon metabolic pathway. This figure also shows where the pathway is impaired for the three starch mutants used in the studies. 1. sex1-1(Starch Excess 1-1) mutant cannot degrade starch efficiently at night and subsequently accumulates a lot of starch in the leaves; 2. adg1-1 (ADP-Glucose pyrophosphorylase) mutant cannot transform Glc-1P into ADP-Glucose; 3. pgm-1 (phosphoglucomutase) mutant cannot transform Glc-6P into Glc-1P. 


\section{Chapter 3}

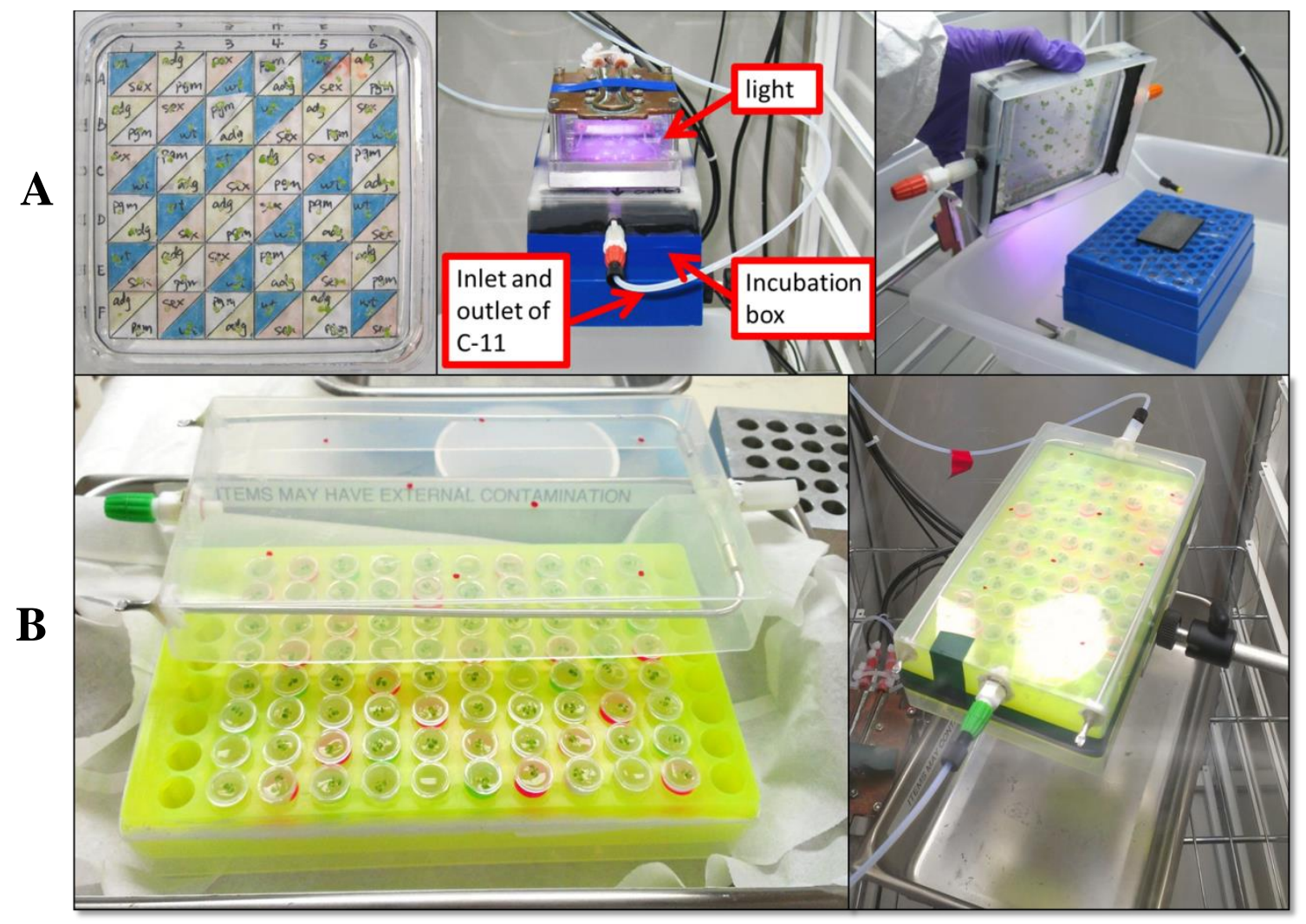

Figure 3-2. The apparati used for ${ }^{11} \mathrm{CO}_{2}$ pulsing and incubation. A: the tip box style incubation chamber for sugar extraction assays; B: the rack style incubation chamber for carbon relocation assays. 

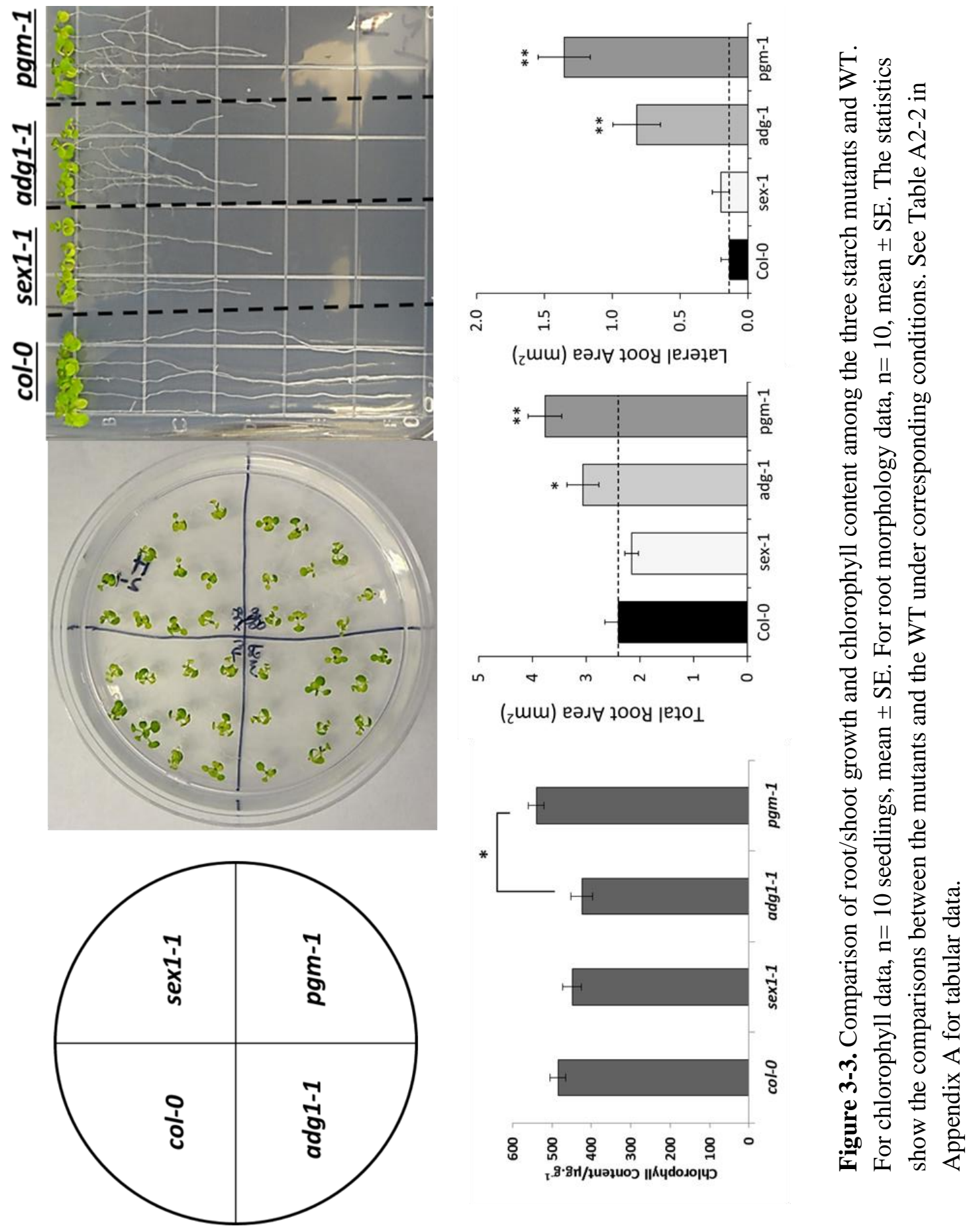


\section{Chapter 359}
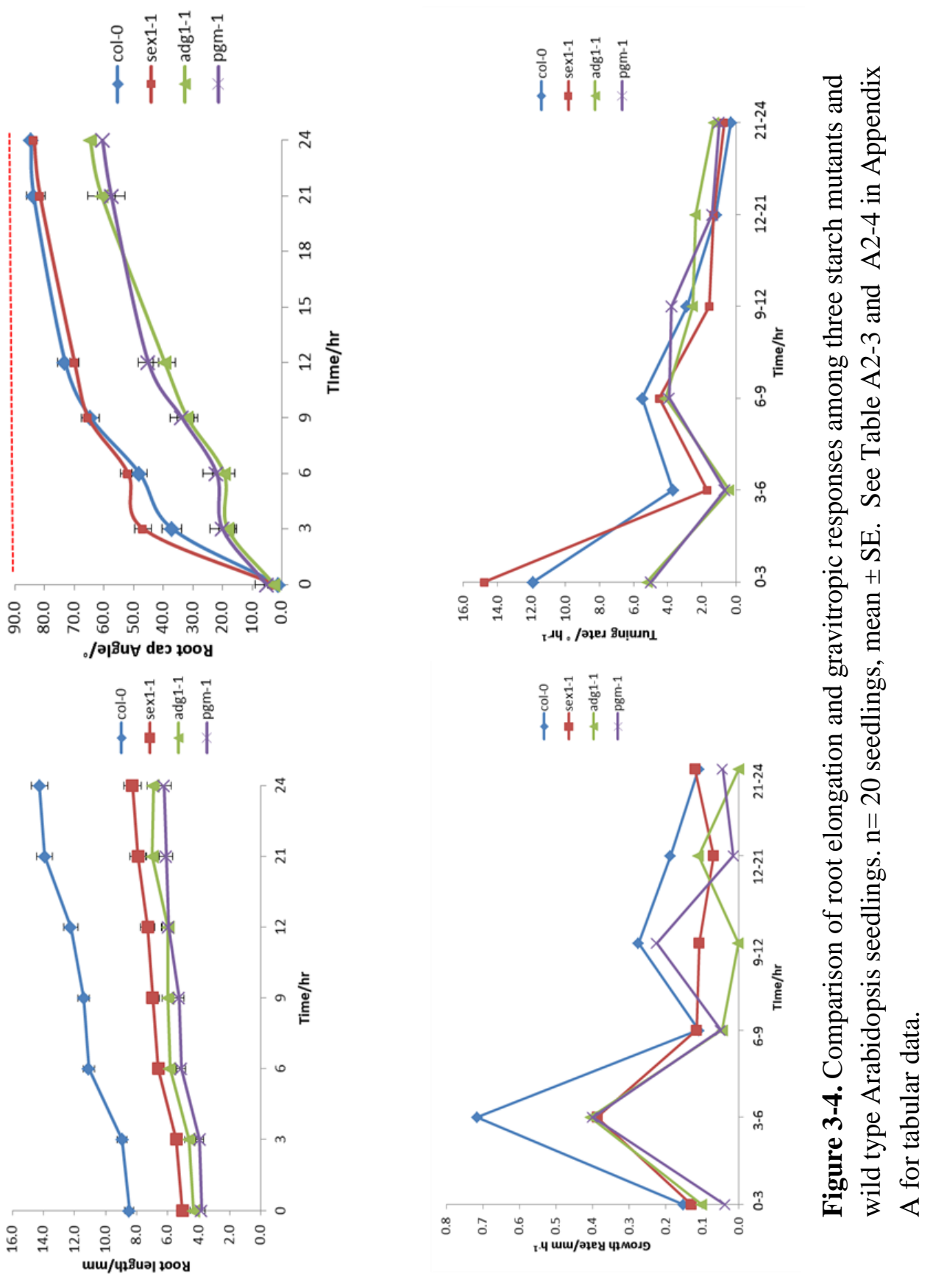


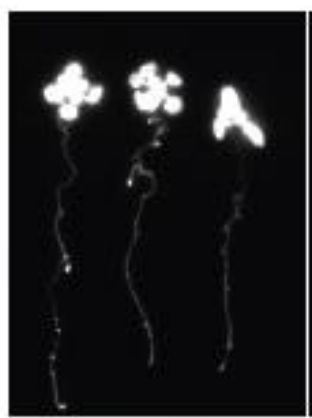

Col-0 (wt)

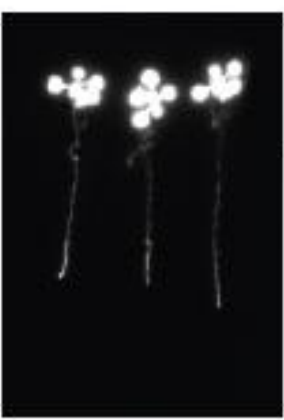

$\operatorname{sex} 1-1$

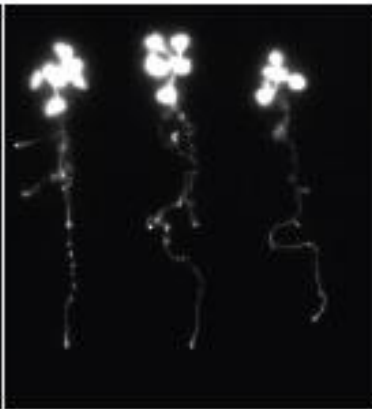

adg1-1

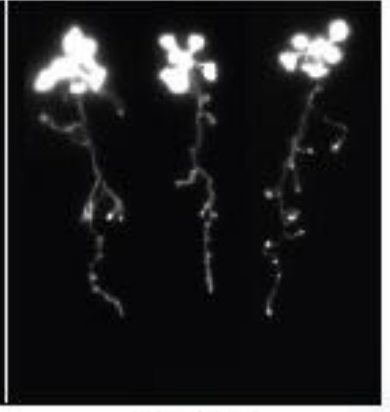

pgm-1

Figure 3-5. Carbon-11 phosphor imaging of the three starch mutants and the wild type. Seedlings were exposed to the phosphor screen 1 hour after the ${ }^{11} \mathrm{CO}_{2}$ pulsing.

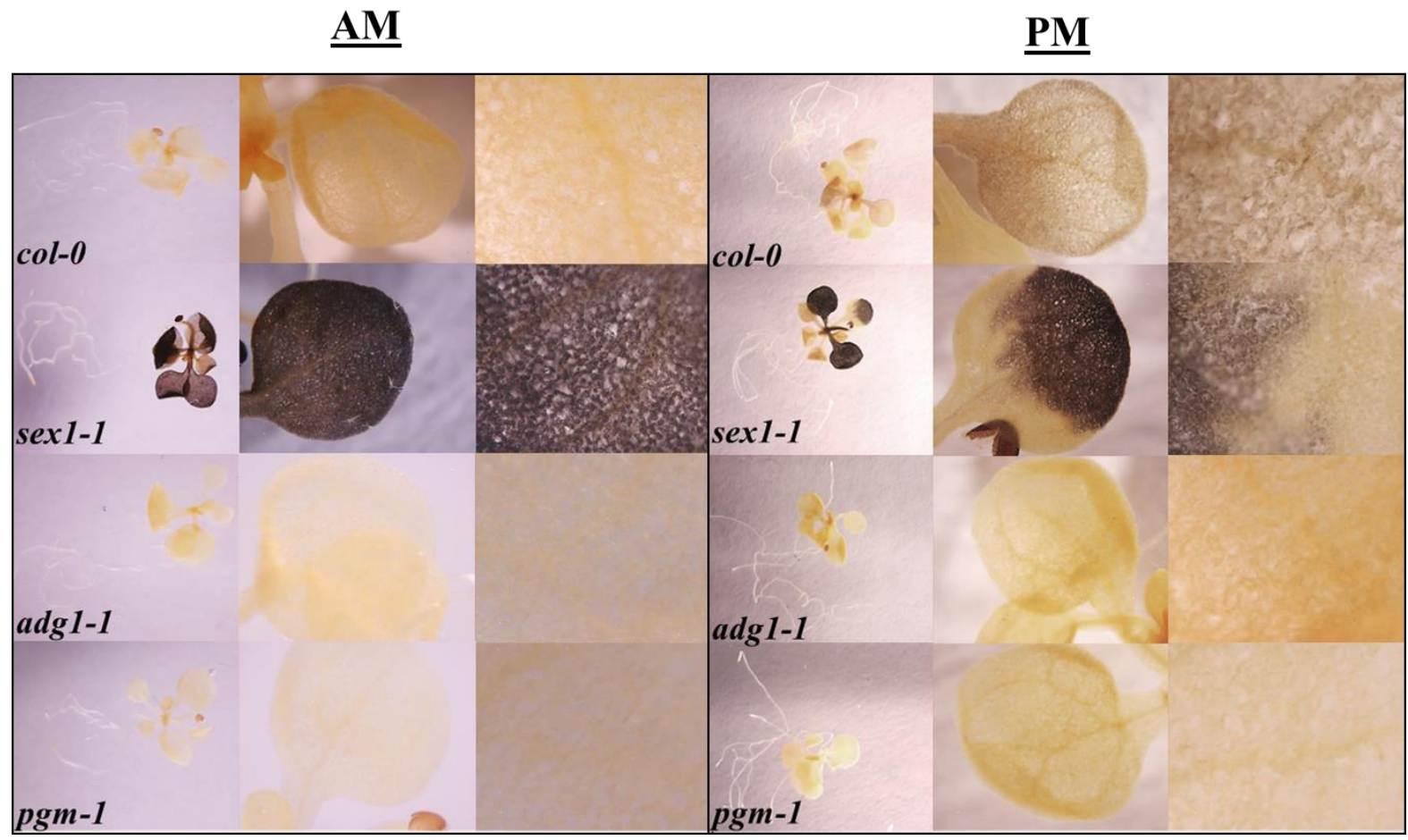

Figure 3-6. Iodine staining assays of starch in three starch mutants and wild type Arabidopsis. The assay was done both in the early morning and late afternoon, labeled as AM and PM. 


\section{Chapter 3}

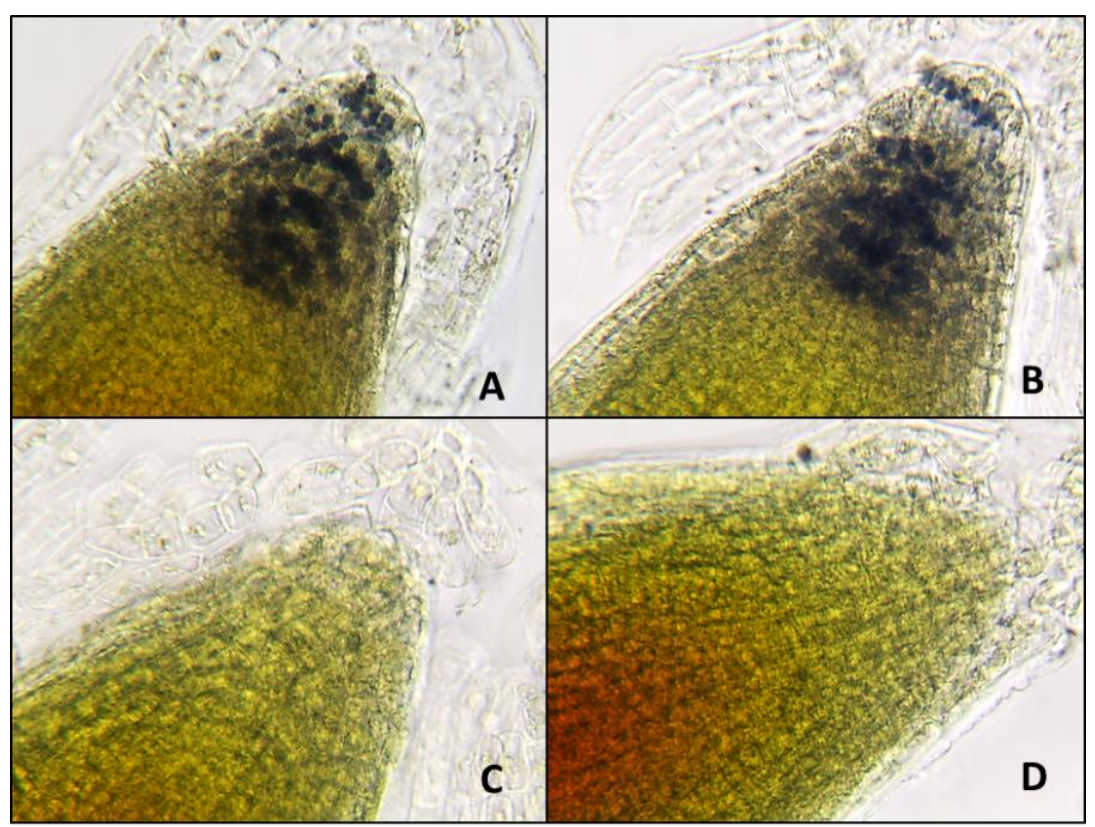

Figure 3-7. Iodine staining assay of starch in the root tips of three starch mutants and wild type Arabidopsis. 

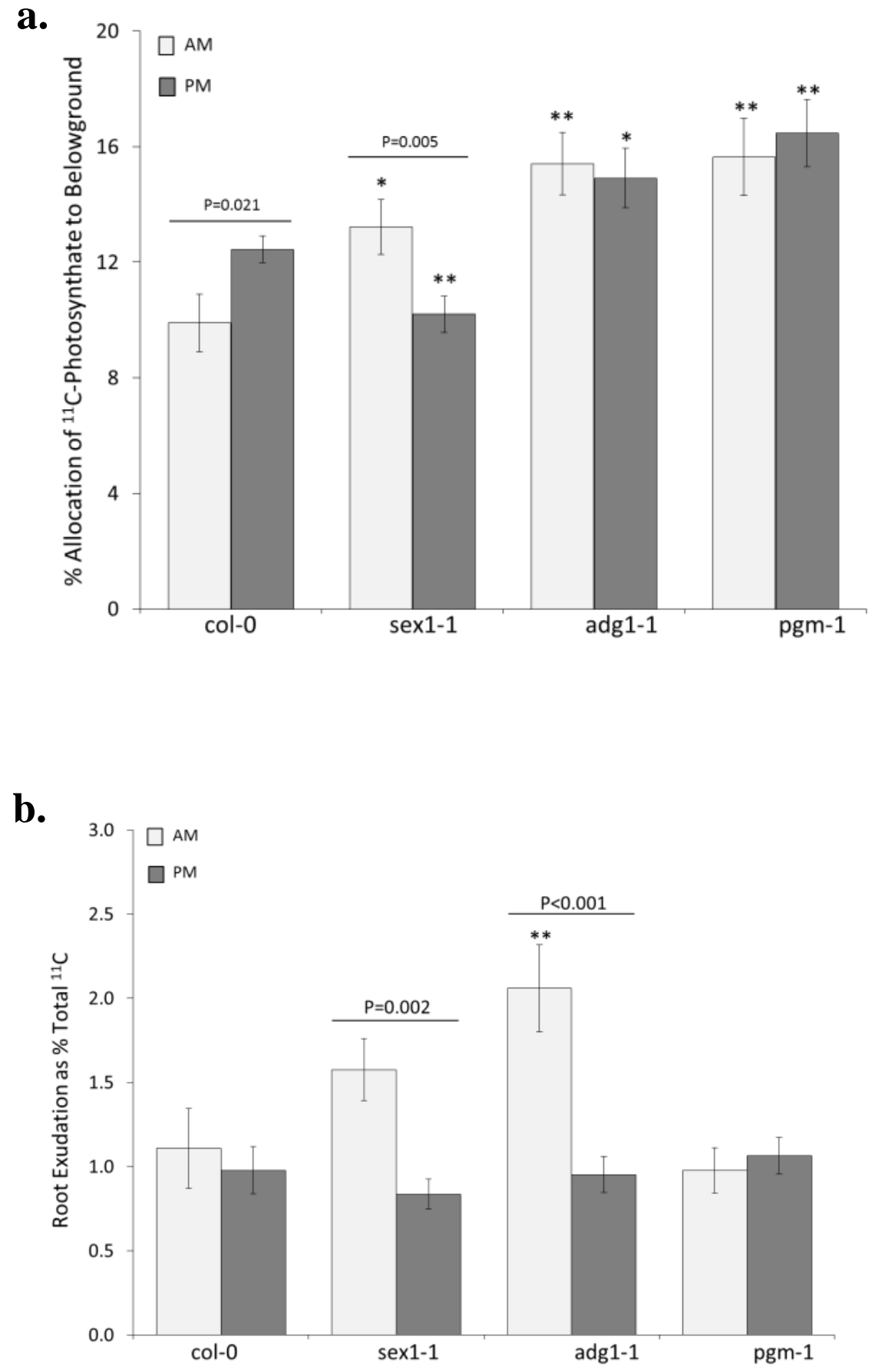

Figure 3-8. ${ }^{11} \mathrm{C}$-carbohydrate relocation to belowground and root exudation. $\mathrm{n}=8-14$ seedlings. The figures show mean $\pm \mathrm{SE}$. The statistics show the comparisons between the mutants and the corresponding wild type. See Table A2-8 in Appendix A for tabular data. 
a
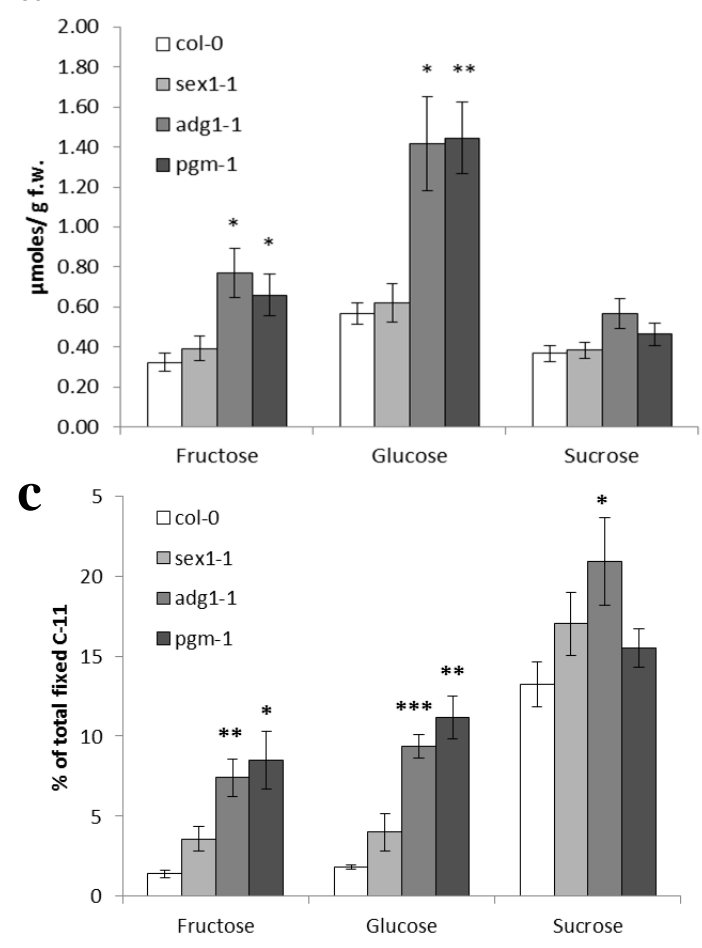

b
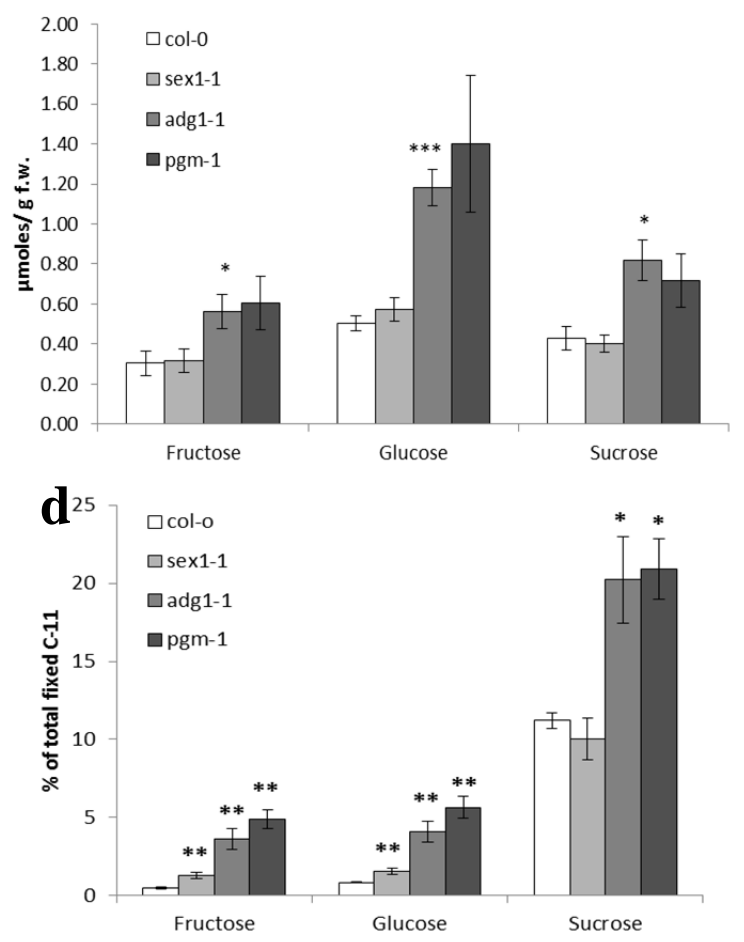

Figure 3-9. ${ }^{12} \mathrm{C}$ and ${ }^{11} \mathrm{C}$ sugar partitioning in the starch mutants and the wild type. a and b, C-12 analysis in AM and PM; c and d, C-11 analysis in AM and PM. Eighteen seedlings of each type of Arabidopsis were combined for sugar extraction and the experiment was repeated 4-5 times. The figures show mean \pm SE. The statistics show the comparisons between the mutants and the corresponding wild type. See Table A2-5 and A2-6 in Appendix A for tabular data. 


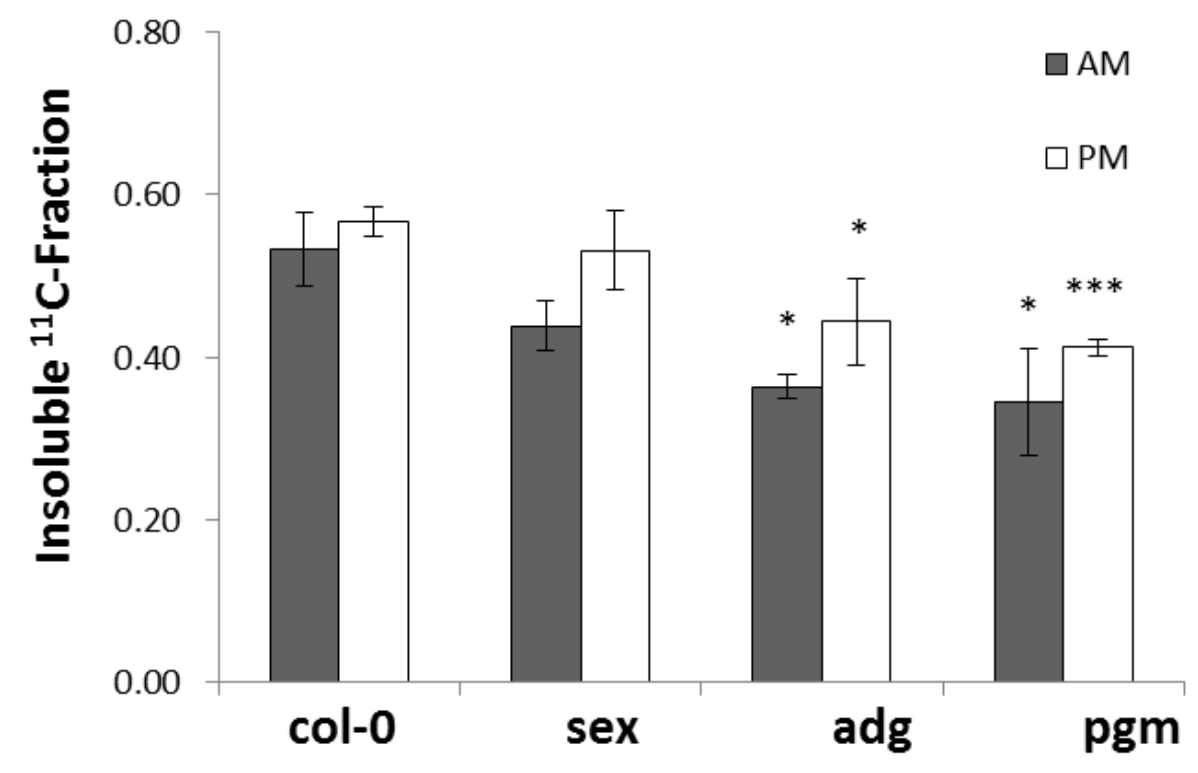

Figure 3-10. Percentage of total fixed ${ }^{11} \mathrm{C}$ assimilated into insoluble carbohydrates. 18 seedlings of each type of Arabidopsis were combined for sugar extraction and the experiment was repeated 4-5 times. The figures show mean $\pm S E$. The statistics show the comparisons between the mutants and the corresponding wild type. See Table A27 in Appendix A for tabular data. 


\section{References}

1. Zimmermann, M., Ziegler, H., List of sugars and sugar alcohols in sieve-tube exudates. Encyclopedia of Plant Physiology, NS, Springer, 1975. 1: p. 480-503.

2. Chia, D.W., et al., Fumaric acid: an overlooked form of fixed carbon in Arabidopsis and other plant species. Planta, 2000. 211(5): p. 743-51.

3. Slewinski, T.L. and D.M. Braun, Current perspectives on the regulation of whole-plant carbohydrate partitioning. Plant Science, 2010. 178(4): p. 341-349.

4. Stettler, M., et al., Blocking the metabolism of starch breakdown products in Arabidopsis leaves triggers chloroplast degradation. Mol Plant, 2009. 2(6): p. 1233-46.

5. Geigenberger, P., Regulation of starch biosynthesis in response to a fluctuating environment. Plant Physiol, 2011. 155(4): p. 1566-77.

6. Zeeman, S.C., S.M. Smith, and A.M. Smith, The diurnal metabolism of leaf starch. Biochem J, 2007. 401(1): p. 13-28.

7. Grennan, A.K., Regulation of starch metabolism in Arabidopsis leaves. Plant Physiol, 2006. 142(4): p. 1343-5.

8. Orzechowski, S., Starch metabolism in leaves. Acta Biochimica Polonica, 2008. 55(3): p. 435-445.

9. Zeeman, S.C. and T.A. Rees, Changes in carbohydrate metabolism and assimilate export in starch-excess mutants of Arabidopsis. Plant, Cell \& Environment, 1999. 22(11): p. $1445-1453$.

10. Schulze, W., et al., Growth and reproduction of Arabidopsis thaliana in relation to storage of starch and nitrate in the wild-type and in starch-deficient and nitrate-uptakedeficient mutants. Plant, Cell \& Environment, 1994. 17(7): p. 795-809.

11. Graf, A., et al., Circadian control of carbohydrate availability for growth in Arabidopsis plants at night. Proc Natl Acad Sci U S A, 2010. 107(20): p. 9458-63.

12. Gibon, Y., et al., Adjustment of growth, starch turnover, protein content and central metabolism to a decrease of the carbon supply when Arabidopsis is grown in very short photoperiods. Plant Cell Environ, 2009. 32(7): p. 859-74. 
13. Smith, A.M. and M. Stitt, Coordination of carbon supply and plant growth. Plant Cell Environ, 2007. 30(9): p. 1126-49.

14. Cross, J.M., et al., Variation of enzyme activities and metabolite levels in 24 Arabidopsis accessions growing in carbon-limited conditions. Plant Physiol, 2006. 142(4): p. 157488.

15. Bahaji, A., et al., Arabidopsis thaliana mutants lacking ADP-glucose pyrophosphorylase accumulate starch and wild-type ADP-glucose content: further evidence for the occurrence of important sources, other than ADP-glucose pyrophosphorylase, of ADPglucose linked to leaf starch biosynthesis. Plant Cell Physiol, 2011. 52(7): p. 1162-76.

16. Caspar, T., S.C. Huber, and C. Somerville, Alterations in Growth, Photosynthesis, and Respiration in a Starchless Mutant of Arabidopsis thaliana (L.) Deficient in Chloroplast Phosphoglucomutase Activity. Plant Physiol, 1985. 79(1): p. 11-7.

17. Periappuram, C., et al., The plastidic phosphoglucomutase from Arabidopsis. A reversible enzyme reaction with an important role in metabolic control. Plant Physiol, 2000. 122(4): p. 1193-9.

18. Yu, T.S., et al., The Arabidopsis sexl mutant is defective in the R1 protein, a general regulator of starch degradation in plants, and not in the chloroplast hexose transporter. Plant Cell, 2001. 13(8): p. 1907-18.

19. Smith, A.M., S.C. Zeeman, and S.M. Smith, Starch degradation. Annu Rev Plant Biol, 2005. 56: p. 73-98.

20. Zeeman, S.C., et al., A starch-accumulating mutant of Arabidopsis thaliana deficient in a chloroplastic starch-hydrolysing enzyme. Plant J, 1998. 15(3): p. 357-65.

21. Trethewey, R.N. and T. ap Rees, A mutant of Arabidopsis thaliana lacking the ability to transport glucose across the chloroplast envelope. Biochem J, 1994. 301 ( Pt 2): p. 44954.

22. Streb, S., et al., The debate on the pathway of starch synthesis: a closer look at lowstarch mutants lacking plastidial phosphoglucomutase supports the chloroplast-localized pathway. Plant Physiol, 2009. 151(4): p. 1769-72.

23. Agtuca, B., Rieger, E., Hilger, K., Song , L., Ferrieri, R.A., Carbon-11 used to Study the Physiological and Metabolic Basis for Hormonal Cross-Talk between Auxin and Salicylic Acid in Maize (Zea mays L.) Root Development. (in review), 2013. 
24. Kazan, K. and J.M. Manners, Linking development to defense: auxin in plant-pathogen interactions. Trends Plant Sci, 2009. 14(7): p. 373-82.

25. Woodward, A.W., Bartel, B., Auxin: Regulation, Action, and Interaction. Annals of Botany, 2005. 95(5): p. 707-735.

26. McSteen, P., Auxin and Monocot Development. Cold Spring Harbor Perspect. Biol 2010. 2: p. 1-17.

27. Ferrieri, R.A., Wolf, A.P., The chemistry of positron emitting nucleogenic atoms with regards to preparation of labeled compounds of practical utility. Radiochimica Acta 1983. 34: p. 69-83.

28. Klaus, R., Fisher, W., Hauck, H.E., Application of a thermal in situ reaction for fluorometric detection of carbhhydrates on NH2-layers. chromatographia, 1990. 29: p. 467-472.

29. Babst B.A., K.A.a.J.T., Biochemical partitioning of recently fixed carbon for studies of rapid plant responses: Radiometabolite analysis of $C-11$ labeled nonstructural carbohydrates. Plant Methods, 2012.

30. Caspar, T., et al., Mutants of Arabidopsis with altered regulation of starch degradation. Plant Physiol, 1991. 95(4): p. 1181-8.

31. Gibon, Y., et al., Adjustment of diurnal starch turnover to short days: depletion of sugar during the night leads to a temporary inhibition of carbohydrate utilization, accumulation of sugars and post-translational activation of ADP-glucose pyrophosphorylase in the following light period. Plant J, 2004. 39(6): p. 847-62.

32. Niittyla, T., et al., A previously unknown maltose transporter essential for starch degradation in leaves. Science, 2004. 303(5654): p. 87-9.

33. Band, L.R., et. al., Root gravitropism is regulated by a transient lateral auxin gradient controlled by a tipping-point mechanism. PNAS, 2012. 109: p. 4668-4673.

34. Caspar, T. and B.G. Pickard, Gravitropism in a starchless mutant of Arabidopsis: implications for the starch-statolith theory of gravity sensing. Planta, 1989. 177: p. 18597.

35. Kiss, J.Z., R. Hertel, and F.D. Sack, Amyloplasts are necessary for full gravitropic sensitivity in roots of Arabidopsis thaliana. Planta, 1989. 177: p. 198-206. 


\section{Chapter 3}

36. Cholodny, N., Wuchshormone und Tropismen bei den Pflanzen. Biol Zentralbl, 1927. 47: p. 604-626.

37. Went, F., Wuchsstoff und Wachstum. Rec Trav BotNeerl, 1928. 25: p. 1-116.

38. Wolverton, C., Paya, A. M., Toska, J., Root cap angle and gravitropic response rate are uncoupled in the Arabidopsis pgm-1 mutant. Physiol Plant, 2011. 141(4): p. 373-82. 


\section{Chapter 4: Effect of Fe status on Carbon Metabolism in Arabidopsis}

\subsection{Isotope Summary}

\begin{tabular}{|c|c|c|c|}
\hline Isotopes & Half-life & Decay Mode & $\mathbf{E}_{\max }$ \\
\hline${ }^{11} \mathrm{C}$ & $20.3 \mathrm{~min}$ & $\beta^{+}(99.8 \%)$, & $0.96 \mathrm{MeV}\left(\beta^{+}\right)$ \\
& & $\mathrm{EC}(0.2 \%)$ & $0.511 \mathrm{MeV}$ annihilation photons $(199.6 \%)$ \\
\hline${ }^{59} \mathrm{Fe}$ & $44.6 \mathrm{~d}$ & $\beta^{-}(100 \%), \gamma$ & $0.273 \mathrm{MeV}(45 \%)$ and $0.466 \mathrm{MeV}(53 \%)\left(\beta^{-}\right) ;$ \\
& & & $1.099 \mathrm{MeV}(57 \%)$ and $1.292 \mathrm{MeV}(43 \%)(\gamma)$ \\
\hline
\end{tabular}

\subsection{Background}

Iron $(\mathrm{Fe})$ is an essential mineral nutrient for plant growth and development. As a co-factor for many vital enzymes [1, 2], including the cytochromes of the electron transport chain [3], it is required for a wide range of biological functions, such as chlorophyll synthesis $[4,5]$, and the maintenance of chloroplast structure and function $[6$, 7]. However, the bio-availability of Fe in the environment varies dramatically, which can affect the normal growth of plants, especially crop species [8, 9]. Iron comprises around $5 \%$ of the earth's crust, but in aerobic alkaline environments, Fe is very limited due to the chemical form of $\mathrm{Fe}(\mathrm{III})$, mainly as oxyhydroxide polymers with extremely low solubility in such environments. The bio-availability of Fe under these conditions is not sufficient to meet plant needs. Plants under Fe deficient conditions can exhibit symptoms like chlorosis in leaves (lack of chlorophyll) and inhibition of root growth. At the other extreme, Fe can be readily available in excess for plants to take up in waterlogged soils due to the low oxygen and low $\mathrm{pH}$ conditions [10]. Excessive Fe content is potentially 
toxic to plants because of the formation of reactive oxygen radicals in cells (Fenton reaction), which can damage important cellular constituents, such as cell membranes. Therefore, the Fe status in plants is tightly controlled. This behavior in plants is called iron homeostasis [11].

The main mechanism to maintain Fe homeostasis is through the regulation of $\mathrm{Fe}$ uptake by plant roots. To avoid excessive uptake of Fe, wetland plants have evolved mechanisms for oxidizing ferrous $\mathrm{Fe}$ in the rhizosphere [12]. To cope with the $\mathrm{Fe}$ limitation in aerobic soils, plants have developed two approaches, referred as Strategy I and Strategy II (Fig. 4-1). Strategy II plants (graminaceous plants), such as grasses, rely on the secretion of phyto-siderophores (PS) and subsequently take up the Fe(III)-PS complex via a trans-membrane transporter, such as the Yellow Stripe1 (YS1) transporter found in maize [13]. Strategy I plants (dicots, non-graminaceous plants), such as Arabidopsis, rely on the induction of a series of physiological responses to assist in the mobilization of the Fe compounds in the environment, such as ATPase-mediated acidification of the rhizosphere [14], up-regulated activity of a plasma membrane-bound ferric reductase (FRO2) [15, 16], and increased expression of an Fe(II) transporter (IRT1) $[15,17,18]$. Impairment of these pathways can disturb the Fe homeostasis in plants and subsequently affect plant growth and development. One Arabidopsis mutant (irt 1-1) that lacks IRT1 expression showed chlorosis and growth inhibition even with sufficient Fe supplies [18]. On the other hand, the Arabidopsis mutant opt3-2 was recently found to over-accumulate $\mathrm{Fe}$ in aerial tissues due to the constitutively up-regulated FRO2 and IRT1 proteins [15, 19], known as the "Fe starvation" response, regardless of the Fe availability in the environment. 
An imbalance of Fe status in plants not only can affect photosynthesis in leaves due its contribution to chlorophyll synthesis, protein synthesis and electron-transfer chain reactions $[3-5,7,20]$, but also can affect carbon partitioning and allocation in both leaves and roots. Carbon metabolism is vital for providing an energy source for plants, contributing to plant skeleton development, and is also involved in metal transportation $[21,22]$, plant long-distance signaling and root exudation. Fe deficiency stress is shown to be associated with enhanced production of organic acids, particularly citric acid, which is a known chelator of $\mathrm{Fe}(\mathrm{III})$ [21]. In the roots of $\mathrm{Fe}$ deficient plants, citrate concentrations can be 3.7- to 8.8-fold higher than the plants with sufficient Fe supplies [22]. Increases in organic acid concentrations in roots of Fe deficient plants are fairly ubiquitous, and occur both in Strategy I and Strategy II plant species. Enhanced $\mathrm{H}^{+}$ exudation in Strategy I plant roots can occur in parallel with increased organic acid under Fe deficient conditions [23]. Sugar metabolism is also found to be altered under Fe deficient conditions in grapevine. Glucose-6-phosphate, fructose-6-phosphate, and 3phosphoglycerate content were found decreased as active Fe decreased in the plant cells [24].

In this study, we investigated the relationship of Fe status and carbon metabolism in two Arabidopsis mutants, opt3-2 and irt1-1. Both of these mutants have malfunctioning Fe regulation systems, but in opposite ways as mentioned above. How the disturbed Fe regulation affects carbon partitioning and allocation was studied using radioactive isotopes of iron and carbon $\left({ }^{59} \mathrm{Fe}\right.$ and $\left.{ }^{11} \mathrm{C}\right)$ to explore the dynamics of $\mathrm{Fe}$ uptake, carbon metabolism and the cross-talk between them in the mutants and the wild type (WT) under different Fe conditions. 


\subsection{Materials and Methods}

\subsubsection{Materials}

Bleach used for seed sterilization was purchased from Pure Bright (Columbia, MO). The $\mathrm{pH}$ indicator, bromocresol purple, was purchased from Thermo Fisher Scientific (New Jersey, USA). ${ }^{59} \mathrm{FeCl}_{3}$ was purchased from Perkin Elmer (Waltham, MA, USA). All other chemicals used in this study were purchased from Sigma Aldrich (St. Louis, MO, USA).

\subsubsection{Plant Growth Conditions}

Seeds were surface-sterilized with a $35 \%$ bleach solution containing $0.1 \%$ Triton X-100 for $15 \mathrm{~min}$, and rinsed several times with sterile deionized water. Prior to germination, seeds were placed at $4^{\circ} \mathrm{C}$ for $2-3$ days to align the seed germination times. Seeds were then germinated and grown in agar medium containing fullstrength modified [-Fe]-Hoagland's solution (5 mM $\mathrm{KNO}_{3}, 5 \mathrm{mM} \mathrm{Ca}\left(\mathrm{NO}_{3}\right)_{2}, 2 \mathrm{mM}$

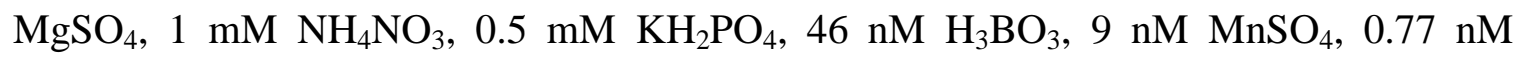
$\mathrm{ZnSO}_{4}, 0.2 \mathrm{nM} \mathrm{CuSO}$ and $0.5 \mathrm{nM} \mathrm{NaMoO}$ ), $0.05 \%$ morpholinoethanesulfonic acid (MES) and $0.5 \%$ agar. $\mathrm{FeCl}_{3}$ solution $(50 \mu \mathrm{M})$ was added to the medium to ensure normal seed germination. The $\mathrm{pH}$ of the medium was adjusted to 6.0 with $5 \mathrm{M} \mathrm{NaOH}$ solution. Seedlings were grown at $22^{\circ} \mathrm{C}$ under $100 \mu$ mole $\mathrm{m}^{-2} \mathrm{~s}^{-1}$ fluorescent white light with a $12 \mathrm{~h}$ (day)/12 h (night) photoperiod. One-week-old seedlings were transferred to a new agar medium for various experimental purposes. For Fe treatment, $\mathrm{FeCl}_{3}$ solution was added to the nutrient medium to reach final concentrations of $9 \mu \mathrm{M}$ (the $\mathrm{Fe}$ 
concentration present in a normal full-strength Hoagland's solution) and $200 \mu \mathrm{M}$. For root analysis, petri dish plates were placed vertically.

\subsubsection{Chlorophyll Content Determination}

Plants were grown under the same conditions as described above in the plant growth conditions. Various concentrations of $\mathrm{FeCl}_{3}(0,9$ and $200 \mu \mathrm{M})$ were added into the growth medium. All the petri dish plates were placed horizontally and the seedlings were grown for 2 weeks in the growth chamber. Eight seedlings were selected from wild type (WT), opt3-2 and irt 1-1 mutant plants grown under different Fe

treatments $\left(0,9\right.$ and $\left.200 \mu \mathrm{M} \mathrm{Fe}^{3+}\right)$. Individual seedlings were put into $1.5 \mathrm{ml}$ Eppendorf tubes and the chlorophyll was then extracted with $1 \mathrm{ml}$ of $100 \%$ methanol. The extract solutions $(200 \mu \mathrm{l})$ were pipetted into 96-well plates and measured using a Bio-TEK plate reader at 652, 665 and $750 \mathrm{~nm}$. Chlorophyll content was calculated using the following equations [25]:

$$
\begin{gathered}
\text { Chl a }=[16.29 \times(\mathrm{A} 665-\mathrm{A} 750)]-[8.54 \times(\mathrm{A} 652-\mathrm{A} 750)] \\
\mathrm{Chl} \mathrm{b}=[30.66 \times(\mathrm{A} 652-\mathrm{A} 750)]-[13.58 \times(\mathrm{A} 665-\mathrm{A} 750)] \\
\mathrm{Chl} \mathrm{a+b}=[22.12 \times(\mathrm{A} 652-\mathrm{A} 750)]+[2.71 \times(\mathrm{A} 665-\mathrm{A} 750)]
\end{gathered}
$$

A652, A655 and A750 indicate the absorbance at 652, 655 and $750 \mathrm{~nm}$ respectively.

Chl a: Chlorophyll a; Chl b: Chlorophyll b; Chl $a+b$ : Chlorophyll a and Chlorophyll b. 


\subsubsection{Proton Concentration Measurements in the Rhizosphere}

The pattern of $\mathrm{pH}$ change in the growth medium was determined by $\mathrm{pH}$ indicator visualization [26]. Each agar plate contained $0.2 \mathrm{mM} \mathrm{CaSO}_{4}$, the $\mathrm{pH}$ indicator bromocresol purple $(0.006 \%)$ and $0.7 \%$ agar. The $\mathrm{pH}$ of the medium was adjusted to 6.0 with $\mathrm{NaOH}$ solution. Seeds were germinated in the agar media with modified [-Fe]Hoagland's solution plus $50 \mu \mathrm{M} \mathrm{FeCl}_{3}$. The addition of $\mathrm{Fe}$ was to ensure normal seed germination. One-week-old seedlings were transferred to agar media with various $\mathrm{Fe}$ concentrations $(0,9$ and $200 \mu \mathrm{M})$ and grown for one week before being transferred to the agar medium containing the $\mathrm{pH}$ indicator. Photographs were taken 24 hours after the transfer.

\subsection{5 ${ }^{59}$ Fe Uptake Assay}

\section{Preparation and Incubation}

Seedlings were grown under the same conditions as mentioned in the Proton Concentration Measurements. Individual seedlings were weighed before being transferred into $1.5 \mathrm{ml}$ centrifuge tubes filled with modified [-Fe]-Hoagland's solution plus the corresponding concentration of Fe. Centrifuge tubes were covered with parafilm, leaving only the shoots of the plants outside (Fig. 4-2), to greatly reduce the possibility of plant shoots contacting the radioactive incubation solution. Syringes and needles were used to introduce the radioactive ${ }^{59} \mathrm{FeCl}_{3}$ solution into the centrifuge tubes. Seedlings were incubated in the radioactive ${ }^{59} \mathrm{FeCl}_{3}$ solution for 2 hours before being harvested. 


\section{Harvesting}

Shoots were removed and placed in counting vials. Roots were removed from the incubation solution, dried, and then placed into $1.5 \mathrm{~mL}$ centrifuge tubes filled with cleaning solution and rinsed by gently vortexing the tubes. This action was repeated three times, twice with the original incubation solution (without ${ }^{59} \mathrm{Fe}$ ) and once with $0.5 \mathrm{mM}$ $\mathrm{CaCl}_{2}$ solution (containing $0.05 \% \mathrm{MES}, \mathrm{pH}$ 6.0). In between each rinse, the roots were dried with KimWipes. Finally, the roots were placed into counting vials. All the samples were counted using liquid scintillation counting (LSC, Tri-Carb 2900TR, Perkin Elmer, Waltham, MA, USA).

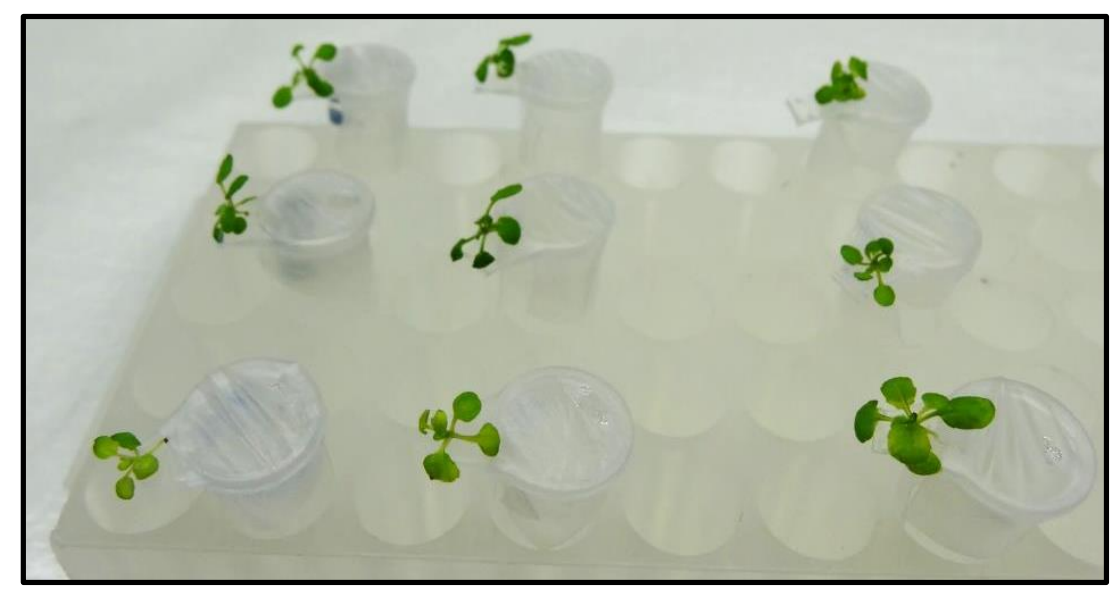

Figure 4-2. Experimental set-up for ${ }^{59} \mathrm{Fe}$ uptake.

\subsection{6 ${ }^{11} \mathrm{CO}_{2}$ production, pulsing and incubation apparatus}

${ }^{11} \mathrm{CO}_{2}$ was produced via the ${ }^{14} \mathrm{~N}(\mathrm{p}, \alpha){ }^{11} \mathrm{C}$ nuclear transformation [27] from a $20 \mathrm{ml}$ target filled with high-purity nitrogen gas (400 mL @ STP) using $18 \mathrm{MeV}$ protons from the TR-19 (Ebco Industries Ltd, Richmond, BC, Canada) cyclotron at Brookhaven National Laboratory (BNL), and captured on molecular sieves $(4 \AA)$. The captured ${ }^{11} \mathrm{CO}_{2}$ 
was desorbed and quickly released into an air stream at $200 \mathrm{~mL} / \mathrm{min}$ as a discrete pulse for labeling the seedlings in different customized sealed incubation chambers (Fig 3-2, Chapter 3). Seedlings within the chamber were pulse-fed ${ }^{11} \mathrm{CO}_{2}$ for 1 minute, then chased with normal air for the duration of the exposure.

Two types of customized apparatus were used as sealed systems during ${ }^{11} \mathrm{CO}_{2}$ pulsing and incubation of the seedlings. For sugar analysis, a square petri dish (without its cover) with seedlings grown in the agar medium was placed within a sealed tip box (Fig. 3-1A). For carbon relocation assays, $1.5 \mathrm{ml}$ Eppendorf ${ }^{\mathrm{TM}}$ tubes with seedlings grown in agar media were placed within a sealed custom fitted tube rack (Fig. 3-1B). Both apparati had two connections, one for ${ }^{11} \mathrm{CO}_{2}$ introduction and one to serve as an exhaust for the closed system. Fixed LED lights were placed on top of the tip box/ tube rack to ensure normal photosynthesis. The ${ }^{11} \mathrm{CO}_{2}$ experiments were conducted both in the early morning ( 1 hour into the start of the photoperiod) and late afternoon ( 7 hours after the AM experiment) as described in the Starch Staining Assay.

\subsubsection{Tissue extraction and soluble sugar analysis}

Seedlings for sugar analysis (wild type, opt3-2 and irt1-1 mutants) were grown on square plates filled with modified [-Fe]-Hoagland's solution plus various concentrations of Fe treatments for 2-3 weeks. After ${ }^{11} \mathrm{CO}_{2}$ pulsing and 20 min of incubation, shoots were harvested and immediately frozen in liquid nitrogen before being ground by hand in pre-weighed and pre-chilled Eppendorf ${ }^{\mathrm{TM}}$ tubes. Four-times the mass of the powder (in $\mathrm{mg}$ ) was introduced as the volume of methanol (in $\mu \mathrm{L}$ ) to the centrifuge tubes, vortexed (VWR analog vortex mixer; Sigma-Aldrich Corp. St. Louis, MO, USA), and then 
sonicated (Branson Bransonic 32; Sigma-Aldrich Corp. St. Louis, MO, USA) at $0{ }^{\circ} \mathrm{C}$ for $10 \mathrm{~min}$. Additional vortexing was performed during this period to ensure complete mixing. The tubes were centrifuged (Eppendorf Centrifuge 5424) for $2 \mathrm{~min}$ at 15,000 rpm to separate the insoluble portion from the soluble one. The insoluble portion contained mostly cell-wall polymers and starch. The soluble portion contained small soluble compounds, including soluble sugars. These sugars were separated and analyzed by thin layer chromatography (TLC) [28, 29]. Glass backed $\mathrm{NH}_{2}$-silica HPTLC-plates $(200 \mu \mathrm{m}$, w/UV254) were used for the sugar separation (Sorbent Technologies, Atlanta, GA, USA). Plates were pre-spotted with $2 \mu \mathrm{L}$ of sugar standards (including 4, 3, 2, 1, 0.5 and $0.2 \mathrm{mM}$ glucose, sucrose and fructose) using a semi-automatic Linomat 5 sample applicator (Camag Scientific Inc., Wilmington, NC, USA) for high precision of the spot size and sample volume. Aliquots $(2 \mu \mathrm{l})$ of leaf extract were also spotted. TLC plates were developed using a mobile phase consisting of 75:25 acetonitrile/water (v/v). The developed TLC plates were imaged using a phosphor imaging screen (FujiFilm BAS-MS 2040, GE Healthcare Biosciences, PA) to determine the yield of each radiolabeled sugar. The plates were then heat-treated $\left(200^{\circ} \mathrm{C}, 10 \mathrm{~min}\right)$ to initiate a chemical reaction of individual sugars with the amino functionalized Si-support giving a fluorescence marker detectable under long wavelength $(365 \mathrm{~nm})$ UV light [28]. Digital photographs were taken of the fluorescent markers to provide a measure of the ${ }^{12} \mathrm{C}$-sugar yields. Image Quant TL software was used to analyze both the radiographic and the digital images to determine the amount of carbon-11 $\left({ }^{11} \mathrm{C}\right)$ and carbon-12 $\left({ }^{12} \mathrm{C}\right)$ sugars. Linear Log regression was used for plotting standard curves generated from the ${ }^{12} \mathrm{C}$-sugar standards. 


\subsubsection{Carbon Allocation}

Seedlings for carbon allocation analysis were grown in square plates for one week, as described in the Tissue Extraction and Soluble Sugar Analysis section. Seedlings were transferred from growth plates into $1.5 \mathrm{ml}$ Eppendorf ${ }^{\mathrm{TM}}$ tubes filled with agar medium containing various concentrations of $\mathrm{FeCl}_{3}$ and allowed to grow for 2-3 weeks. After ${ }^{11} \mathrm{CO}_{2}$ pulsing and 1 hour incubation, the entire seedlings were removed from the agar medium and cut into roots and shoots, then stored in respective vials filled with methanol. Tissues were later counted in a NaI well-counter. The remaining tubes containing agar medium were also placed individually into empty vials for counting, which indicated the amount of ${ }^{11} \mathrm{C}$-labeled root exudates. This experiment was conducted both in the early morning and late afternoon as mentioned in the ${ }^{11} \mathrm{CO}_{2}$ production, pulsing and incubation apparatus.

The percentages of ${ }^{11} \mathrm{C}$-phytosynthates transported to belowground and exuded from the roots were calculated as follows:

$$
\begin{gathered}
\% \text { of }{ }^{11} \text { C-phytosynthates below }=\frac{A_{\text {roots }}+A_{\text {exudates }}}{A_{\text {shoots }}+A_{\text {roots }}+A_{\text {exudates }}} \times 100 \% \\
\% \text { of }{ }^{11} \text { C-phytosynthates exudates }= \\
A_{\text {exudates }}
\end{gathered}
$$

$\mathrm{A}_{\text {shoots, }} \mathrm{A}_{\text {roots }}$ and $\mathrm{A}_{\text {exudates }}$ indicate the activities measured in the shoots, root tissues and in the gel (root exudates). 


\subsubsection{Phosphor imaging}

After the Carbon Allocation assay, some of the seedlings were removed from the $1.5 \mathrm{ml}$ Eppendorf ${ }^{\mathrm{TM}}$ tubes and exposed to a phosphor imaging screen (FujiFilm, BAS-MS grade) for 20 seconds, which was then analyzed using a phosphor-imager Typhoon $^{\mathrm{TM}}$ FLA 9000, GE Healthcare).

\subsubsection{Statistical Analysis}

Data was subjected to the Student t-test for unpaired samples assuming an unequal variance. Statistical significance levels were assigned to the following rating scale $(*, p<0.05 ; * *, p<0.01 ; * * *, p<0.001)$.

\subsection{Results}

\subsubsection{Plant Growth}

The opt3-2 and irt1-1 mutants and the WT Arabidopsis seedlings were grown under three different Fe conditions: no Fe, sufficient Fe $(9 \mu \mathrm{M})$ and excess Fe $(200 \mu \mathrm{M})$. The shoot/root growth and the chlorophyll content of the mutants and the WT Arabidopsis seedlings were compared.

The WT seedlings had low chlorophyll content and inhibited root growth under Fe deficient conditions and showed increased chlorophyll content and improved shoot/root growth under Fe sufficient and Fe excess conditions. There was no significant change in the growth of the WT seedlings between Fe sufficient and Fe excess conditions. 
The opt3-2 and irt1-1 mutants showed severe chlorosis and inhibited shoot/root growth under Fe deficient condition, with the irt $1-1$ mutant seedlings more affected (Fig. 4-3). When sufficient Fe was present in the media, the opt3-2 mutant seedlings exhibited increased levels of chlorophyll content and improved shoot/root growth compared to the ones under Fe deficient conditions. The irt1-1 mutant showed equal levels of severe chlorosis and inhibited shoot/root growth under the sufficient Fe conditions, as under the Fe deficient conditions. However, under Fe excess conditions, the irt1-1 mutant seedlings showed significant improvements in growth, with bigger shoots and longer roots, in comparison to both the WT and the opt3-2 mutant. The chlorophyll content in the irt1-1 mutant was also increased more under Fe excess conditions than under Fe sufficient conditions, although it was still lower than observed with the WT and opt3-2 mutant.

\subsubsection{Acidification of Rhizosphere through Root exudation}

Each type of Arabidopsis seedling had a unique response to the varying levels of Fe availability, with regard to the $\mathrm{pH}$ measurement assays of the root exudates. Seedlings were originally grown in the media at $\mathrm{pH}$ 6.0. All three types of Arabidopsis seedlings showed rhizosphere acidification ( $\mathrm{pH}$ 5.0-5.5) around the roots under Fe deficient conditions (Fig. 4-4B). With sufficient $\mathrm{Fe}^{3+}(9 \mu \mathrm{M})$ present in the media, all three types showed increased $\mathrm{pH}(\mathrm{pH}$ 6.5-7.0) in areas surrounding the roots, while the opt $3-2$ and irt1-1 mutants continued to exhibit acidification around the roots (Fig. 4-4C). With excess $\mathrm{Fe}^{3+}(200 \mu \mathrm{M})$ present in the medium, the WT and the opt3-2 mutant showed only a slight change in the $\mathrm{pH}$ of root exudates, whereas the irt $1-1$ mutant exhibited low $\mathrm{pH}$ 
areas around the roots (Fig. 4-4D). The increasing $\mathrm{pH}$ observed around the roots is likely due to $\mathrm{NO}_{3}{ }^{-}$supplied in the medium as a nitrogen source [30].

\subsection{3 ${ }^{59}$ Fe Uptake under Various Levels of External Fe Availability}

The ${ }^{59} \mathrm{Fe}$ uptake assay reports the amount of Fe taken up into a seedling within a 2-hour period (Fig. 4-5). With sufficient Fe $(9 \mu \mathrm{M})$ available in the environment, the WT tended to take up $45 \%$ more Fe than the opt $3-2$ and 52\% more than the irt $1-1$ mutants.

With excess Fe present in the environment, both the WT and the opt3-2 mutants showed around twice as much of the Fe uptake as the irt $1-1$ mutant did. The irt $1-1$ mutant consistently showed low Fe uptake levels due to a lack of the IRT1 Fe transporter regardless of the external $\mathrm{Fe}$ availability. Data for ${ }^{59} \mathrm{Fe}$ uptake in the three Arabidopsis genotypes under Fe deficient conditions are not shown here because the seedlings were too weak to survive the 2 hours incubation.

\subsubsection{Carbon Fixation, Metabolism and Allocation}

The ${ }^{11} \mathrm{CO}_{2}$ fixation data showed that there was no significant change in carbon fixation levels in the WT seedlings when they were grown under Fe deficient or Fe excess conditions (Fig. 4-6). The opt3-2 mutant had similar levels of carbon fixation as the WT. However, the irt $1-1$ mutant showed a dramatic decrease in carbon fixation compared to the WT when grown under Fe deficient conditions. For example, the irt $1-1$ mutant only fixed $19000 \mathrm{nCi}$ of $\mathrm{C}-11$ whereas the WT fixed around $45000 \mathrm{nCi}$ of $\mathrm{C}-11$. As the amount of Fe present in the environment increased, the irt1-1 mutant showed improved carbon fixation and reached a level similar to the WT. 
The levels of ${ }^{11} \mathrm{CO}_{2}$ fixation in both mornings and afternoons were analyzed. Under Fe deficient conditions, the carbon fixation levels of the three types of seedlings did not change between the morning and afternoon. Under Fe sufficient and Fe excess conditions, both the WT and the irtl-1 mutant showed decreased levels of carbon fixation in the afternoon. Taking the carbon fixation under Fe sufficient conditions as an example, the level in the WT decreased from $40000 \mathrm{nCi}(\mathrm{AM})$ to $30000 \mathrm{nCi}(\mathrm{PM})$, whereas the level in the irt1-1 mutant decreased from $40000 \mathrm{nCi}(\mathrm{AM})$ to $26000 \mathrm{nCi}(\mathrm{PM})$. The opt32 mutant showed no change in carbon fixation level between morning and afternoon.

Our ${ }^{11} \mathrm{C}$ metabolic data (Fig. 4-7) demonstrated that the opt3-2 and irt 1-1 mutants tended to assimilate ${ }^{11} \mathrm{C}$ to high percentages of glucose in the leaves, especially under $\mathrm{Fe}$ sufficient and Fe excess conditions, which was observed in both mornings and afternoons. The opt3-2 mutant also showed a high percentage of glucose under Fe deficient conditions during the morning (opt3-2 vs. WT, $6.9 \%$ vs. $5.4 \%$ ) but not the afternoon (opt3-2 vs. WT, $4.7 \%$ vs. $4.5 \%$ ).

Sucrose was shown to be the primary form of soluble sugars and sucrose synthesis showed a different pattern from glucose (Fig. 4-7B). The opt3-2 mutant exhibited a similar percentage of sucrose as the WT did in the leaves regardless of the Fe availability in the environment. However, the irt1-1 mutant showed a significantly lower percentage of sucrose than the WT (irtl-1 vs. WT, $30.7 \%$ vs. $41.7 \%$ in the morning and $18.0 \%$ vs. $32.5 \%$ in the afternoon) under Fe deficient conditions and exhibited an increase when more Fe was present in the environment, from $30.7 \%$ (Fe deficient) to $37.8 \%$ (Fe excess) in the morning and from $18.0 \%$ (Fe deficient) to $36.7 \%$ (Fe sufficient) and $26.1 \%$ (Fe excess) in the afternoon. Under Fe excess conditions, the percentages of 
sucrose in the form of soluble sugars in the irt1-1 mutant reached the same levels as in the WT (irt $1-1$ vs. WT, $37.8 \%$ vs. $33.7 \%$ in the morning and $26.1 \%$ vs. $24.0 \%$ in the afternoon). Our results also showed that under Fe sufficient conditions, all three types of Arabidopsis seedlings showed a higher percentage of sucrose in the afternoon (an increase from $24.5 \%$ to $36.6 \%$ in the irt $1-1$ mutant), while under Fe deficient or Fe excess conditions, a significantly reduced sucrose fraction was found in all the seedlings (a decrease from $30.7 \%$ to $18.0 \%$ in the irt $1-1$ mutant under Fe deficient conditions).

Our carbon allocation data indicated that the WT had 2-4\% of ${ }^{11} \mathrm{C}$-phytosynthates transported belowground under Fe deficient conditions and exhibited an increase to around $6 \%$ in the morning when more Fe was present in the environment (Fig. 4-8A). Compared to the WT, the opt3-2 and the irt $1-1$ mutants exhibited high percentages of ${ }^{11} \mathrm{C}$-phytosynthates in the belowground portion regardless of the Fe availability in the environment. Our results also showed that all seedlings tended to transport relatively less

${ }^{11} \mathrm{C}$-phytosynthates to the belowground in the afternoon (Fig 4-8B).

In addition, our root exudation data showed that the WT Arabidopsis had reduced root exudation under Fe stress conditions, while the irt 1-1 mutant constantly had relatively high root exudation. The opt3-2 mutant behaved similarly to the WT, but only showed increased root exudation under Fe excess conditions (Fig. 4-9A). Root exudations were dramatically reduced in the afternoon for all the seedlings (Fig. 4-9B).

\subsection{Discussion}

Previous research has shown the Fe uptake ability and Fe regulation in the opt3-2 and irt 1-1 mutants and all of the studies focus on Fe status in mature plants, with most of 
the results based on a long-term Fe accumulation process [15, 31]. Vert's study [32] used

${ }^{55} \mathrm{Fe}$ as a radiotracer to track the Fe status in the $\operatorname{irt} 1-1$ mutant. However, the plants in his study required 2 days of incubation, which is still a cumulative result. In our study, ${ }^{59} \mathrm{Fe}$ was used to investigate the total Fe uptake of the entire plant within hours. Also, seedlings were used to test if they exhibit the same behavior as the mature plants.

Our ${ }^{59} \mathrm{Fe}$ uptake results showed that, within the short incubation time window, different Fe uptake abilities among mutants and the WT were observed, especially under Fe excess conditions (Fig. 4-5). The irt 1-1 mutant took up less ${ }^{59}$ Fe than the WT under both Fe sufficient (6000 cpm/mg vs. $9000 \mathrm{cpm} / \mathrm{mg}$ ) and Fe excess conditions (4000 cpm/mg vs. $10000 \mathrm{cpm} / \mathrm{mg})(9 \mu \mathrm{M}$ and $200 \mu \mathrm{M})$, whereas the opt3-2 mutant showed similar levels of ${ }^{59} \mathrm{Fe}$ as the WT did under all Fe treatments. Previous studies have shown that the opt3-2 mutant plants over-accumulate Fe in the aerial tissues; however, this phenotype was not seen in our ${ }^{59} \mathrm{Fe}$ uptake experiments, where plants were only incubated in the radioactive ${ }^{59} \mathrm{FeCl}_{3}$ solution for 2 hours. This result indicates that $\mathrm{Fe}$ over-accumulation phenotype of the opt3-2 mutant is very likely a result of long-term regulation, rather than a kinetically rapid Fe uptake. (This behavior is also seen in heavy metal accumulation in the opt3-2 mutant. See Chapter 2).

Iron plays key roles in many biological processes. Plant growth and development can be significantly affected under long-term Fe stress (deficiency or toxicity). The most common symptom under Fe deficiency is leaf chlorosis. This is because the precursor of chlorophyll and heme synthesis is aminolevulinic acid (ALA), and the rate of ALA synthesis is controlled by Fe [4]. Fe can also affect chloroplast development due its contributions to many chloroplastic proteins and RNA syntheses, as well as its role in the 
electron transport chain. Under Fe deficient conditions, less Fe is present in the cells, which can limit all of the bio-processes mentioned above and result in a chlorosis phenotype.

The WT Arabidopsis seedlings in our study showed lower chlorophyll content under Fe deficiency conditions than under Fe sufficient and Fe excess conditions (Fig. 43). However, our ${ }^{11} \mathrm{C}$ data showed no significant change in carbon fixation in the WT under any Fe treatments (Fig. 4-6). This result indicated a disconnection between chlorophyll content and photosynthesis (i.e., it could be positively or negatively correlated), which was also found in previous studies [33-35]. It is possible that the decreased level of chlorophyll content in the WT was not significant enough to affect carbon fixation.

The iron over-accumulation phenotype in the opt3-2 mutant can promote the production of reactive oxygen species, which are radicals that can damage the regular biological activities in plant cells, especially photosynthetic machinery in the chloroplasts. Our ${ }^{11} \mathrm{C}$ data showed that, compared to the WT, although the opt3-2 mutant exhibited a relatively high level of carbon fixation under Fe sufficient conditions, a decrease was observed under Fe excess conditions (Fig. 4-6). However, the Fe overaccumulation in the opt3-2 mutant did not inhibit chlorophyll synthesis in the mutant (Fig. 4-3), which indicated that high Fe levels in plants is more likely to cause damage in carbon fixation, rather than chlorophyll synthesis.

The irt 1-1 mutant plants, with excess Fe present in the environment, showed significant increases in chlorophyll content and shoot size, compared to itself under $9 \mu \mathrm{M}$ 
$\mathrm{Fe}^{3+}$ treatment, although the irt1-1 mutant plants do not have an efficient Fe transporter (IRT1) in roots $[32,36]$. This result is consistent with the previous studies [32]. It was shown that IRT1 is not the only iron transporter in root cells. IRT2 is also found in Arabidopsis roots and up-regulated as part of the "Fe starvation" response although it is not used as a major Fe transporter [37]. This contributes to the phenotype mentioned above. The shoot growth of the irt $1-1$ showed a dramatic improvement, even having a larger size than the WT under the same Fe conditions (Fig. 4-3). The mechanism for this phenotype is still unclear. The carbon fixation level in the irtl-1 mutant showed a positive correlation with chlorophyll content (Fig. 4-6), which supported that an Fe deficient status in plants can significantly affect chlorophyll synthesis and subsequently carbon fixation levels.

In addition to the effects on shoot growth, the internal Fe status can also affect root growth. Fe deficiency is associated with inhibition of root elongation, increases in the diameter of apical root zones, and abundant root hair formation [13, 38, 39]. Our results showed that, under Fe deficient conditions, the irt1-1 mutant exhibited the most severe inhibition of root growth compared to the other two types (Fig. 4-3). With addition of $9 \mu \mathrm{M} \mathrm{Fe}^{3+}$, the root growth of all types of Arabidopsis seedlings improved notably, with the irt $1-1$ mutant still exhibiting much shorter roots than the other two types. The irt 1-1 mutant showed dramatic improvements in root growth with an excess of Fe supply. Like the shoot growth, the mechanism of this phenotype for the irt $1-1$ mutant is still unknown.

Plant Fe status can also cause a redirection of carbon partitioning and carbon allocation. Iron deficiency has generally been shown to cause increases in the organic 
acid concentrations, such as citrate, in roots, stem exudates and leaves of different plant species $[22,40]$. This shift in carbon usage can indirectly affect the carbon metabolic pathways, such as sugar synthesis, which also can be affected by photosynthesis. In our

${ }^{11} \mathrm{C}$ experiments, glucose tended to be elevated in Fe-deficient plants (Fig. 4-7A), whereas the sucrose level in the irtl-1 mutant was much lower than in the other two types of Arabidopsis and this trend only occurred under low Fe concentrations (Fig. 4-7B). This might be due to reduced carbon fixation ability (Fig. 4-6) as well as high levels of carbon allocation to roots (Fig. 4-8).

Our ${ }^{11} \mathrm{C}$ data showed that $\mathrm{Fe}$ status could alter carbon allocation and carbon metabolism in plants and subsequently affect root exudation. Under Fe deficient conditions, the opt3-2 and the irt1-1 mutants transported higher percentages of ${ }^{11} \mathrm{C}$ photosynthates to the belowground compared to the WT. However, when more Fe was added to the medium, this trend disappeared (Fig. 4-8A) This increased transport of photosynthates to belowground in the mutants under Fe deficiency stress is possibly due to a high demand for carbon in the roots for either enhanced root growth to reach out for more Fe, or to synthesize Fe-stress related molecules, such as organic acids or chelators. The data are not normalized by mass, so the increasing trend for the WT and the opt3-2 mutant under Fe sufficient and Fe excess conditions may be partially due to the increasing root/mass ratio. However, the opt3-2 and irt $1-1$ mutants under Fe deficient conditions showed high photosynthates present in roots even though they did not have high root/shoot ratios (Fig. 4-3). Furthermore, our root exudation data indicated that the irt1-1 had much higher amounts of carbohydrates exuded from the roots under $\mathrm{Fe}$ deficiency stress. Also, the root exudation $\mathrm{pH}$ data showed that the irt $1-1$ exhibited 


\section{Chapter 4}

rhizosphere acidification under Fe deficient conditions (Fig. 4-4). Therefore, the combination of these two results suggests that, besides $\mathrm{H}^{+}$exudation, the irt $1-1$ mutant is likely to have elevated amounts of organic acids synthesized, which are transported to roots and secreted to the rhizosphere to increase Fe acquisition ability. To verify the hypothesis, root exudates need to be analyzed in the future.

In addition, we also found that the amount of carbon allocated to belowground and secreted out of the roots was notably reduced in the afternoon for all types of Arabidopsis under all Fe treatments. Therefore, this behavior is very likely a general regulation pattern found in plants.

In this project, using ${ }^{59} \mathrm{Fe}$ - and ${ }^{11} \mathrm{C}$ as radiotracers has provided us an opportunity to not only individually study the dynamic properties of the metabolism and allocation of $\mathrm{Fe}$ and $\mathrm{C}$ within a short-time window, but also explore the relationships between $\mathrm{Fe}$ homeostasis and carbon metabolism and allocation among the three types of Arabidopsis. However, further investigations (root exudate analysis) and experimental repetitions (the

${ }^{59} \mathrm{Fe}$ uptake assays) are still needed in the future to better understand the cross-talk between iron homeostasis and carbon metabolism. 


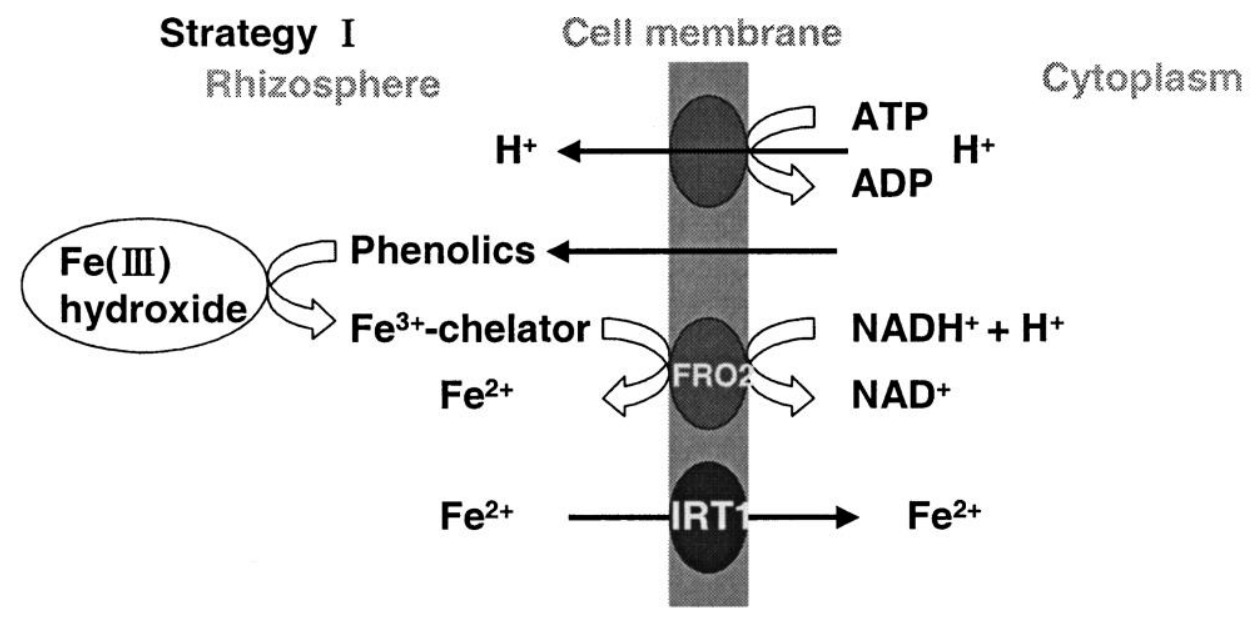

Strategy II

Rhizosphere

Cell membrane

Cytoplasm

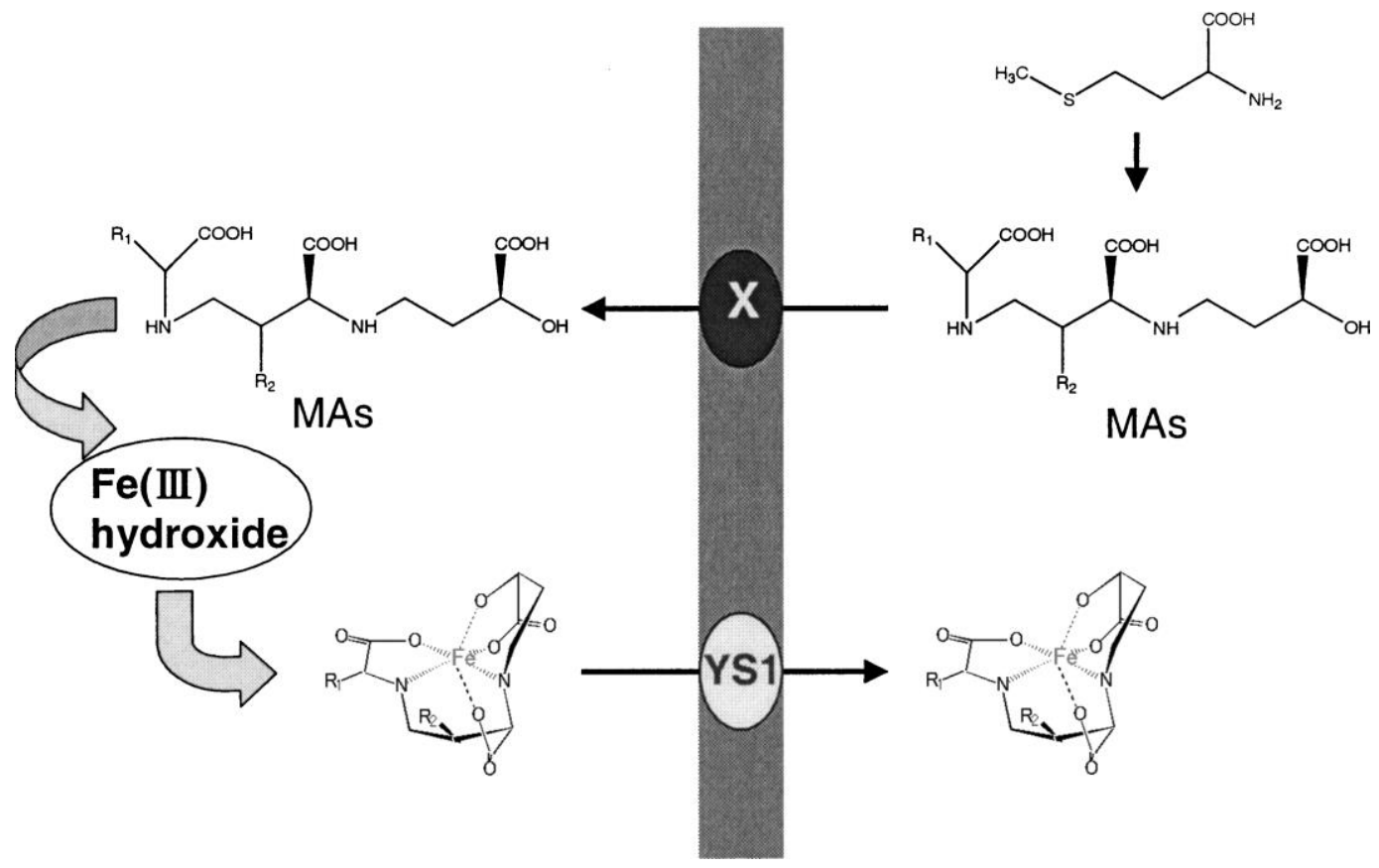

Figure 4-1. Two strategies for iron uptake in plants [41]. Strategy I is used by monocot and nongraminaceous plants and Strategy II is used by graminaceous plants. FRO, Ferric-chelate Reductase; IRT, Iron Regulated Transporter; YS, Yellow Stripe. 

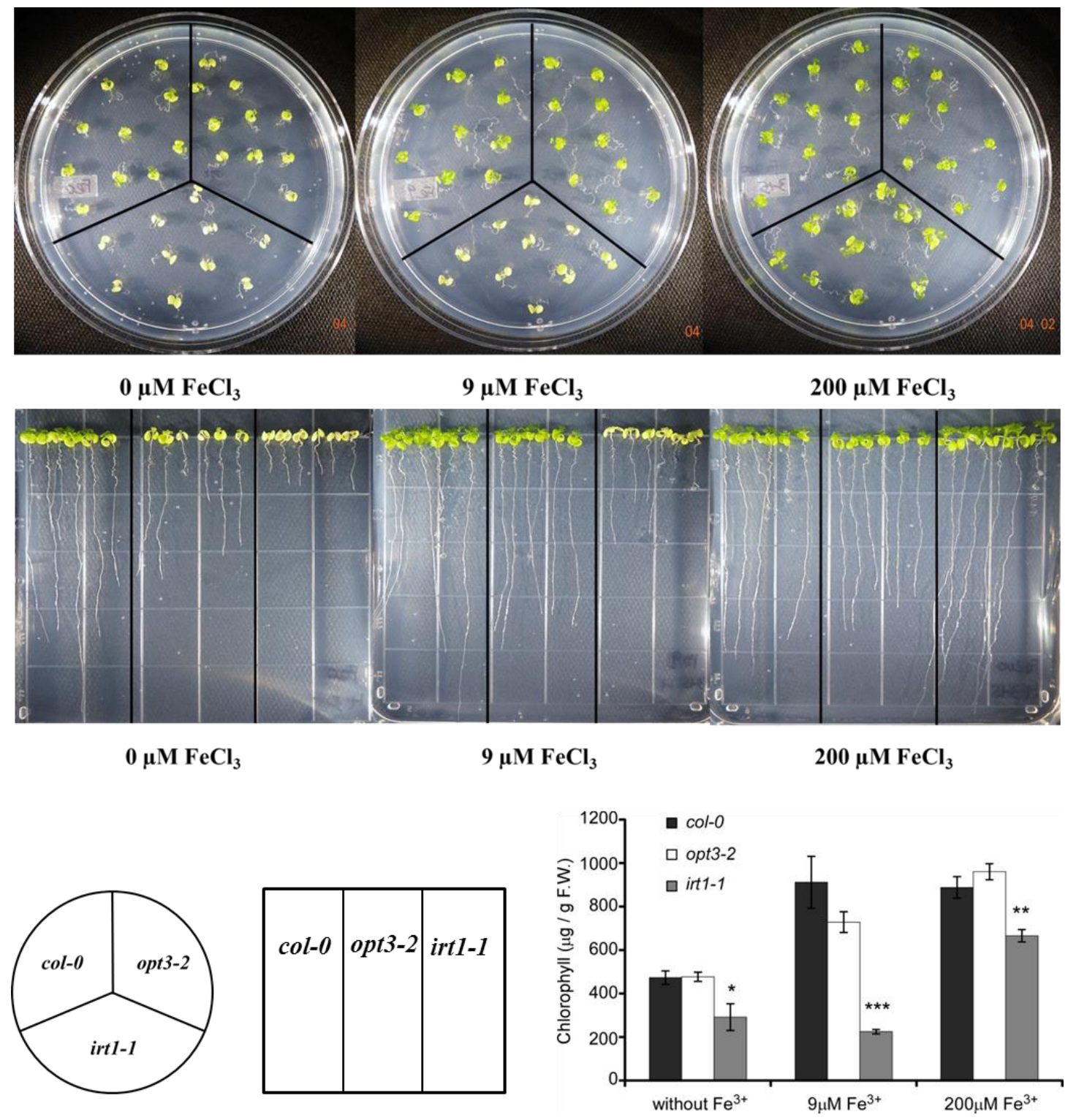

Figure 4-3. Comparison of root/shoot growth and chlorophyll content among the mutants and WT. For chlorophyll data, $n=8$ seedlings, mean \pm SE. The statistics show the comparisons between the mutants and the WT under corresponding conditions. The tabular data is listed in Table 3-1 in Appendix A. 


\section{Chapter 4}

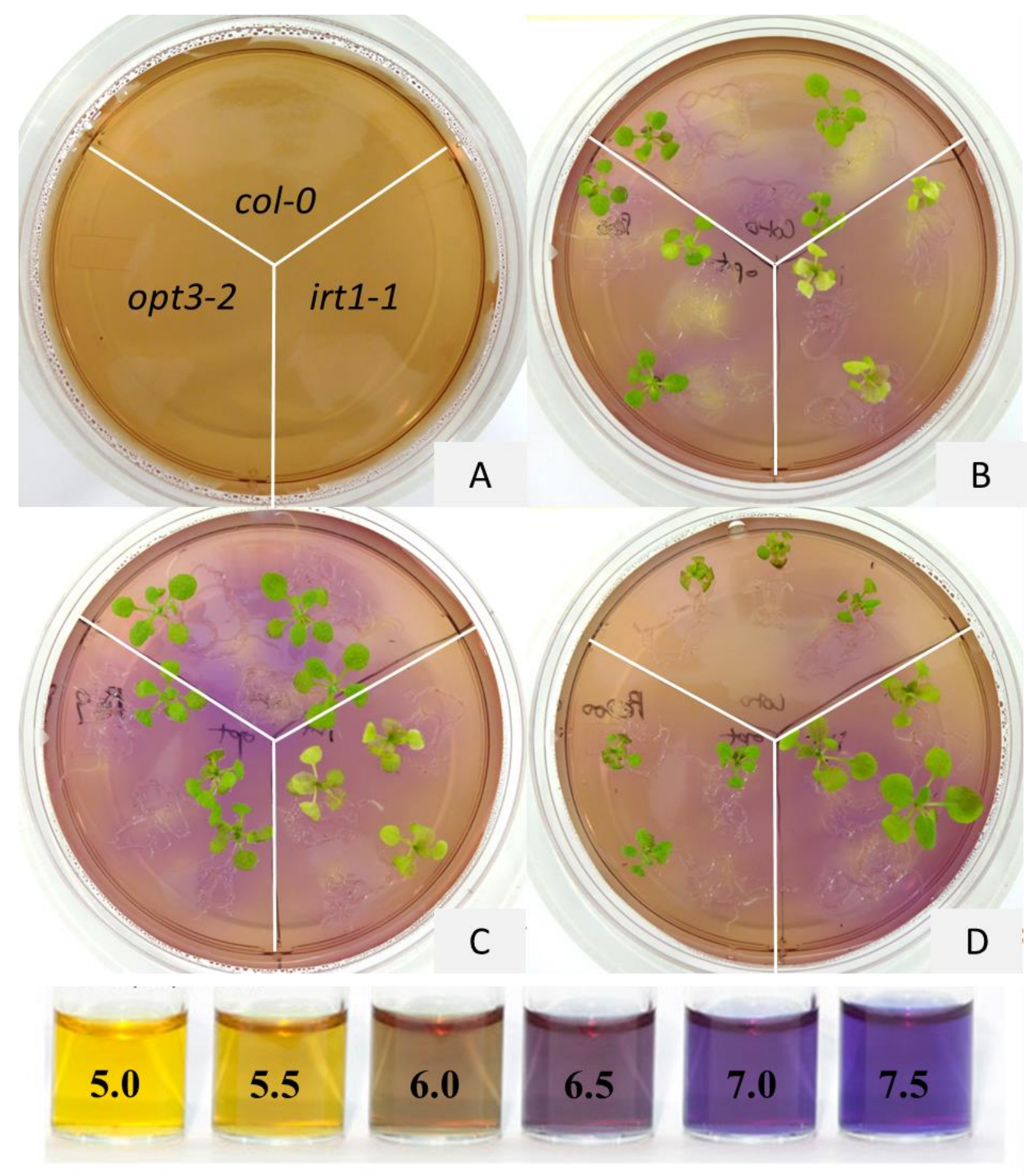

Figure 4-4. Measurement of root exudate $\mathrm{pH}$ of Arabidopsis seedlings (wt, opt3-2and irt1-1) grown under various concentrations of $\mathrm{Fe}$ treatments using a $\mathrm{pH}$ indicator. $\mathbf{A}$, blank; $\mathbf{B}$, without $\mathrm{Fe}$; C, with $9 \mu \mathrm{M} \mathrm{Fe}^{3+} ; \mathbf{D}$, with $200 \mu \mathrm{M} \mathrm{Fe}^{3+}$. 


\section{Chapter 492}

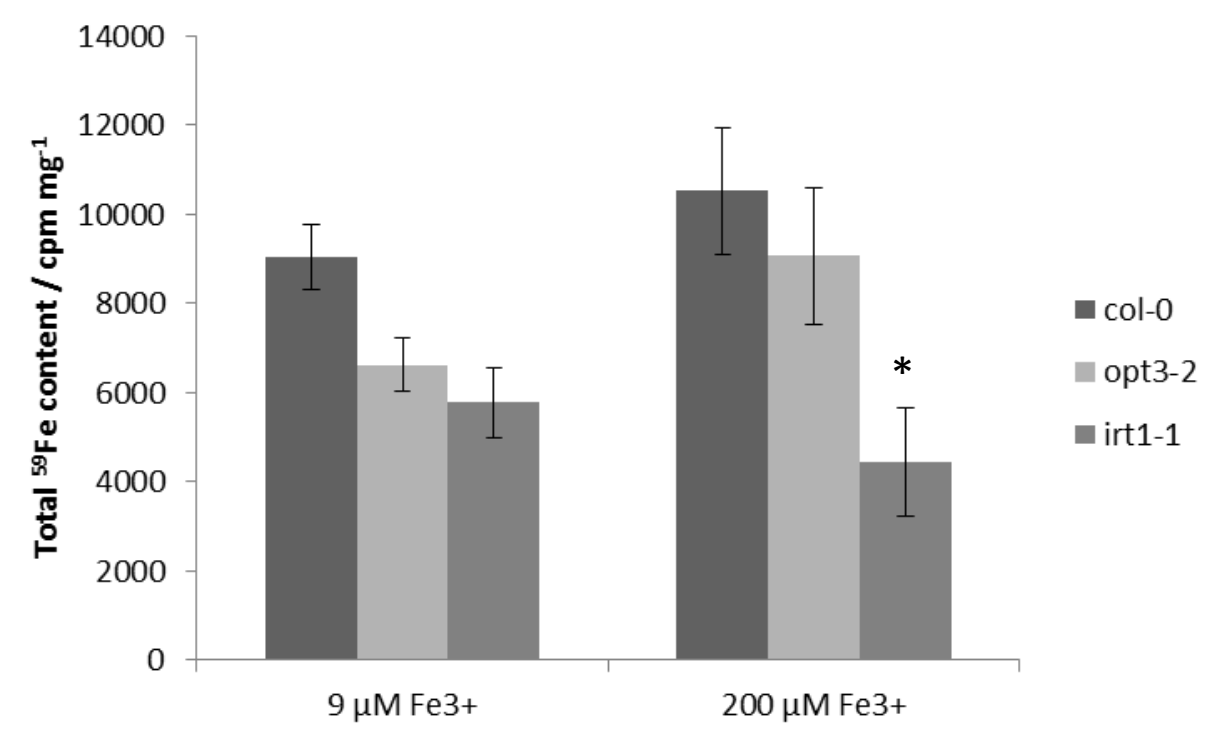

Figure 4-5. ${ }^{59} \mathrm{Fe}$ uptake under various levels of external Fe availability. $n=8-9$ seedlings, mean \pm SE. The statistics show the comparisons between the mutants and the WT under corresponding conditions. Tabular data is presented in Table A3-2 in Appendix A. 


\section{A}

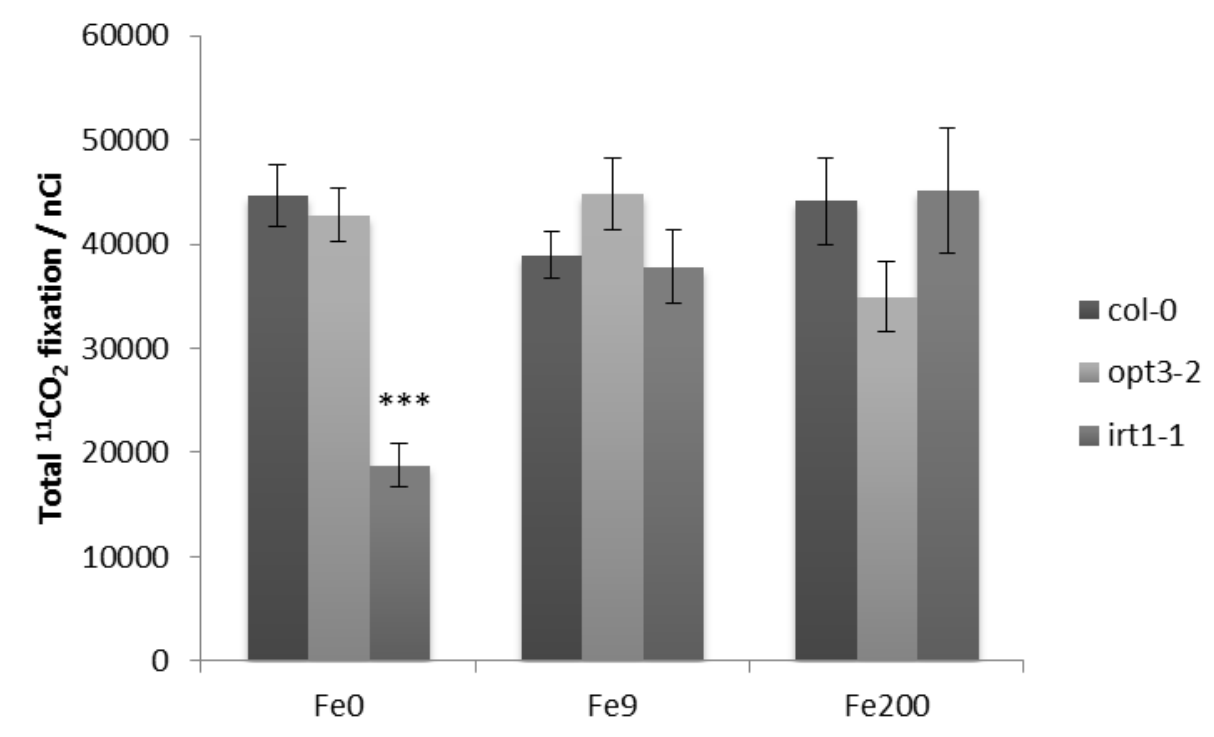

B

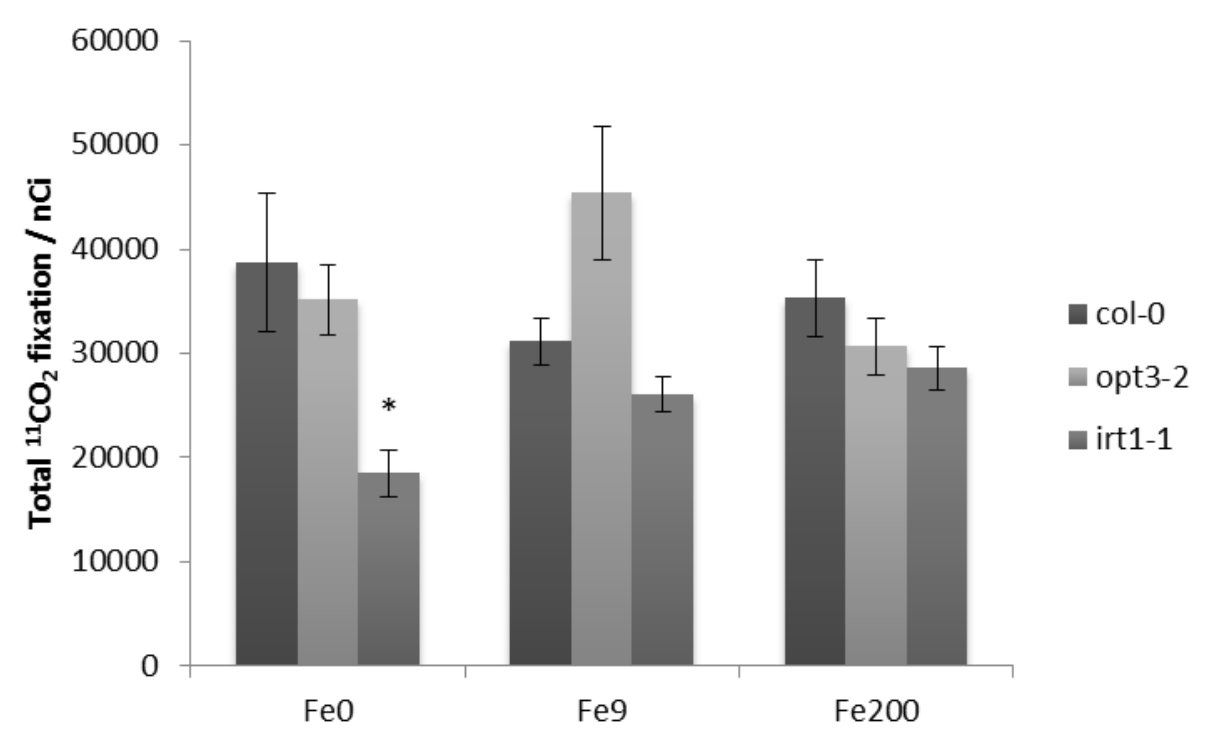

Figure 4-6. Total ${ }^{11} \mathrm{CO}_{2}$ fixation. The experiments were conducted both in the morning (A) and afternoon (B). $n=6-8$ seedlings. The statistics show the comparisons between the mutants and the WT under corresponding conditions. Tabular data is listed in Table A3-3 in Appendix A. 

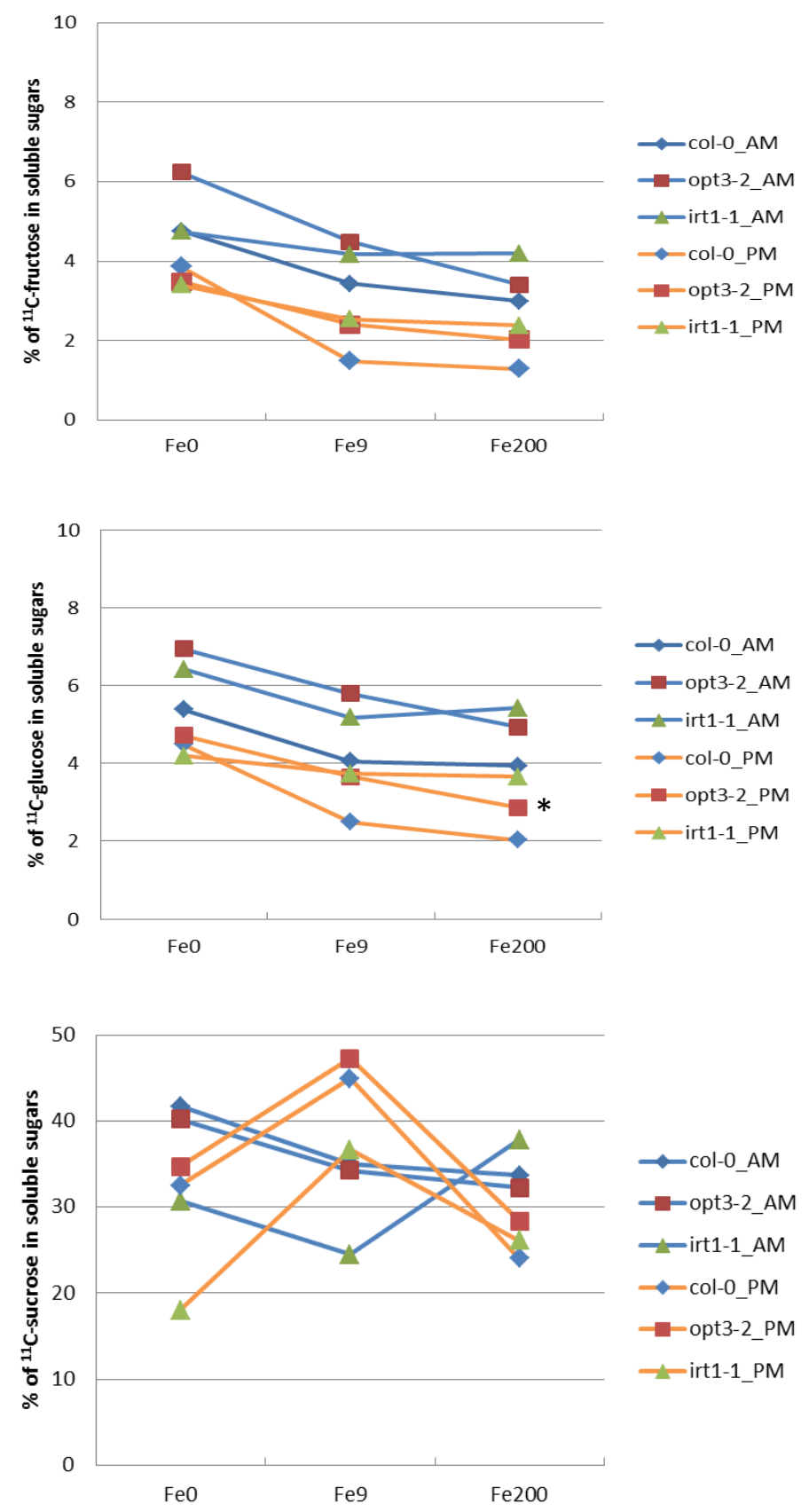

Figure 4-7. ${ }^{11} \mathrm{C}$-sugar partitioning in the leaves of three types of Arabidopsis seedlings. Fructose, glucose and sucrose were extracted from leaf tissue and analyzed. This assay was conducted in both morning (blue) and afternoon (orange). Eighteen seedlings of each type of Arabidopsis were combined for the sugar extractions and the experiments were repeated 3-4 times. The figures show mean $\pm \mathrm{SE}$. The statistics show the comparisons between the mutants and the corresponding wild type. The same data plotted as bar graphs is shown in Figure A 3-2, A3-3 and A3-4 in Appendix A. Tabular data is given in Table A3-4 in Appendix A. 


\section{Chapter 495}
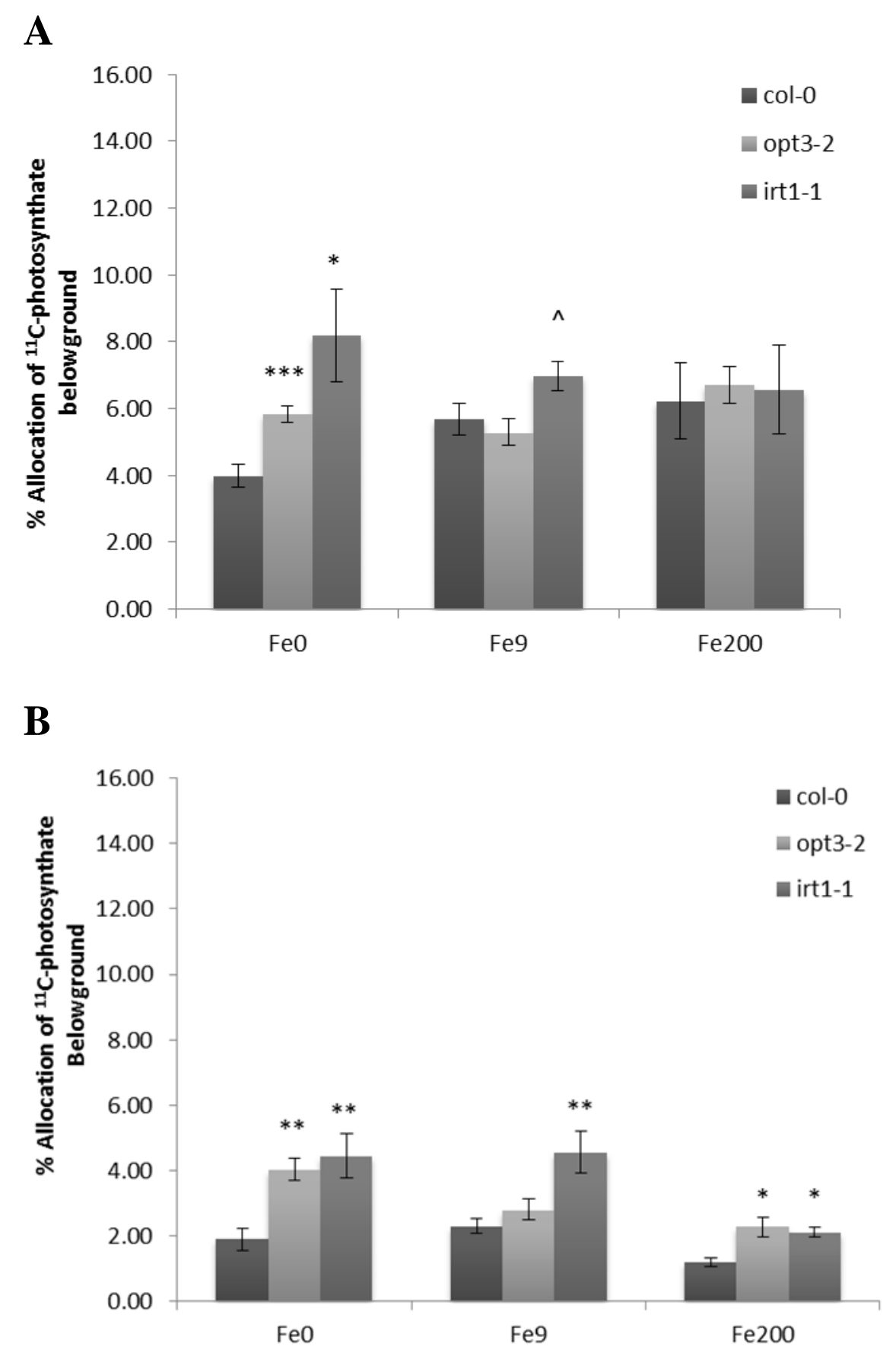

Figure 4-8. Allocation of ${ }^{11} \mathrm{C}$-photosynthates to the belowground. The experiments were conducted both in the morning (A) and afternoon (B). The figures show mean $\pm S E, n=6-8$ seedlings. The statistics show the comparisons between the mutants and the WT under corresponding conditions. Tabular data is presented in Table A3-5 in Appendix A. 

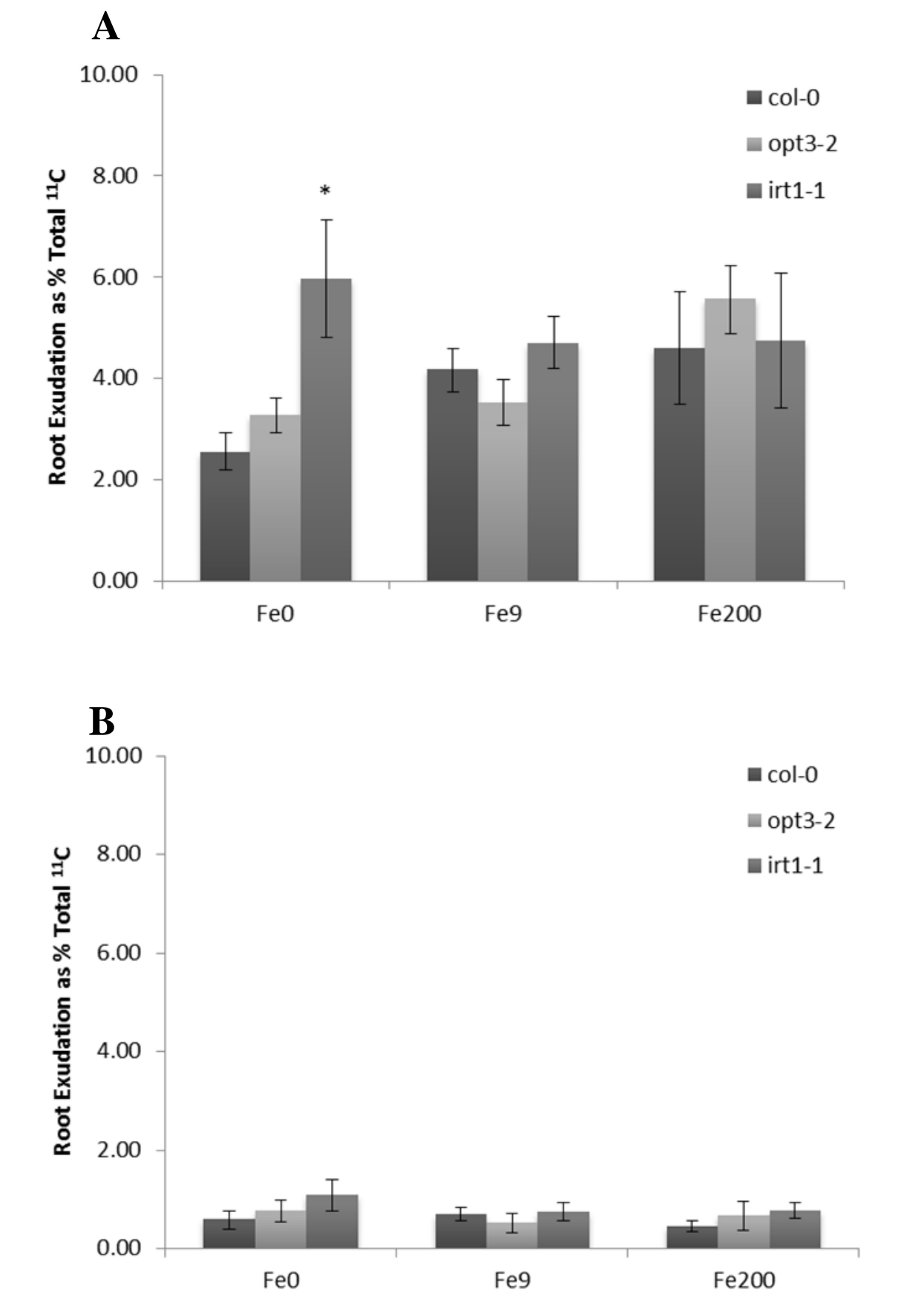

Figure 4-9. Root exudation. The experiments were conducted both in the morning (A) and afternoon (B). $n=6-8$ seedlings. The statistics show the comparisons between the mutants and the WT under corresponding conditions. Tabular data is given in Table A3-5 in Appendix A. 


\section{References}

1. Fridovich, I., Superoxide radical: an endogenous toxicant. Annu. Rev. Pharmacol. Toxicol., 1983. 23: p. 239-257.

2. Hildebrand, D., Lipoxygenases. Physiol. Plant, 1989. 76: p. 249-253.

3. Powers, L., et al., Structural features and the reaction mechanism of cytochrome oxidase: iron and copper X-ray absorption fine structure. Biophysical Journal, 1981. 34(3): p. 465-498.

4. Pushnik, J.C., Miller, G.W., Iron regulation of chloroplast photosynthetic function: mediation of PSI development. J. Plant Nutr., 1989. 12: p. 407-421.

5. Spiller, S.C., Castelfranco, A. M., Castelfranco, P. A., Effects of Iron and Oxygen on Chlorophyll Biosynthesis: I. In Vivo Observations on Iron and Oxygen-Deficient Plants. Plant Physiology, 1982. 69(1): p. 107-111.

6. Abadia, J., Leaf responses to Fe deficiency: a review. Journal of Plant Nutrition, 1992. 15: p. 1699-1713.

7. Pushnik, J., Miller, GW and Manwaring, JH, The role of iron in higher plant chlorophyll biosynthesis, maintenance and chloroplast biogenesis. Journal of Plant Nutrition, 1984. 7: p. 733-758.

8. Berman-Frank, I., et. al., Iron availability, cellular iron quotas, and nitrogen fixation in Trichodesmium. . Limnol. Oceanogr., 2001. 46: p. 1249.

9. Yamauchi, M., Rice Bronzing in Nigeria Caused by Nutrient Imbalances and its Control by Potassium Sulfate Application. Plant Soil, 1989. 117: p. 275-286.

10. Janiesch, P., Ecophysiological adaptations of higher plants in natural communities to waterlogging. Miscellaneous Ecological responses to environmental stresses, 1991: p. 5060.

11. Jeong, J. and M.L. Guerinot, Homing in on iron homeostasis in plants. Trends Plant Sci, 2009. 14(5): p. 280-5.

12. Schmidt, W., Mechanisms and regulation of reduction-based iron uptake in plants. New Phytol, 1999. 141: p. 1-26. 


\section{Chapter $4 \quad 98$}

13. Romheld, V.M., H, Mobilization of Iron in the Rhizosphere of Different Plant Species. Advances in in Plant Nutrition, 1986: p. 95-124.

14. Toulon, V., et al., Role of apoplast acidification by the H+ pump. Planta, 1992. 186(2): p. 212-218.

15. Stacey, M.G., et al., The Arabidopsis AtOPT3 protein functions in metal homeostasis and movement of iron to developing seeds. Plant Physiol, 2008. 146(2): p. 589-601.

16. Robinson, N.J., et al., A ferric-chelate reductase for iron uptake from soils. Nature, 1999. 397(6721): p. 694-7.

17. Fox, T.C., et al., Direct Measurement of 59Fe-Labeled Fe2+Influx in Roots of Pea Using a Chelator Buffer System to Control Free Fe2+ in Solution. Plant Physiol, 1996. 111(1): p. 93-100.

18. Eide, D., et al., A novel iron-regulated metal transporter from plants identified by functional expression in yeast. Proc Natl Acad Sci U S A, 1996. 93(11): p. 5624-8.

19. Stacey, M.G., et al., AtOPT3, a member of the oligopeptide transporter family, is essential for embryo development in Arabidopsis. Plant Cell, 2002. 14(11): p. 2799-811.

20. Lin, C.H. and C.R. Stocking, Influence of leaf age, light, dark, and iron deficiency on polyribosome levels in maize leaves. Plant and Cell Physiology, 1978. 19(3): p. 461-470.

21. de Vos, C.R., H.J. Lubberding, and H.F. Bienfait, Rhizosphere Acidification as a Response to Iron Deficiency in Bean Plants. Plant Physiology, 1986. 81(3): p. 842-846.

22. Abadía, J., et al., Organic acids and Fe deficiency: a review. Plant and Soil, 2002. 241(1): p. 75-86.

23. Bienfait, H.F., Is there a metabolic link between $H+$ excretion and ferric reduction by roots of Fe-deficient plants? - a viewpoint. Journal of Plant Nutrition, 1996. 19(8-9): p. 1211-1222.

24. Smith, B.R. and L. Cheng, Iron Assimilation and Carbon Metabolism in 'Concord' Grapevines Grown at Different pHs. Journal of the American Society for Horticultural Science, 2007. 132(4): p. 473-483. 
25. Porra, R.J., Thompson W.A., Kreidemann, P.E., Determination of Accurate Extinction Coefficients and Simultaneous Equations for Assaying Chlorophylls a and b Extracted with Four Different Solvents: Verification of the Concentration of Chlorophyll Standards by Atomic Absorption Spectroscopy. 1989, Biochimica et Biophysica Acta. p. 348-389.

26. Yi, Y. and M.L. Guerinot, Genetic evidence that induction of root Fe(III) chelate reductase activity is necessary for iron uptake under iron deficiency. Plant J, 1996. 10(5): p. 835-44.

27. Ferrieri, R.A., Wolf, A.P., The chemistry of positron emitting nucleogenic atoms with regards to preparation of labeled compounds of practical utility. Radiochimica Acta 1983. 34: p. 69-83.

28. Klaus, R., Fisher, W., Hauck, H.E., Application of a thermal in situ reaction for fluorometric detection of carbhhydrates on NH2-layers. chromatographia, 1990. 29: p. 467-472.

29. Babst B.A., K.A.a.J.T., Biochemical partitioning of recently fixed carbon for studies of rapid plant responses: Radiometabolite analysis of $C-11$ labeled nonstructural carbohydrates. Plant Methods, 2012.

30. Marschner, H. and V. Römheld, In vivo Measurement of Root-induced pH Changes at the Soil-Root Interface: Effect of Plant Species and Nitrogen Source. Zeitschrift für Pflanzenphysiologie, 1983. 111(3): p. 241-251.

31. Vert, G., et al., IRT1, an Arabidopsis transporter essential for iron uptake from the soil and for plant growth. Plant Cell, 2002. 14(6): p. 1223-1233.

32. Vert, G., et al., IRT1, an Arabidopsis Transporter Essential for Iron Uptake from the Soil and for Plant Growth. The Plant Cell Online, 2002. 14(6): p. 1223-1233.

33. Fleischer, W.E., The Relation Between Chlorophyll Content and Rate of Photosynthesis. J Gen Physiol, 1935. 18(4): p. 573-97.

34. Kirchhoff, W.R., et. al., Inheritance of a mutation influencing chlorophyll content and composition in cowpea. Crop Science, 1989. 29: p. 105-108.

35. Pettigrew, W.T., et al., Characterization of Canopy Photosynthesis of ChlorophyllDeficient Soybean Isolines. Crop Sci., 1989. 29(4): p. 1025-1029. 
36. Vert, G.A., J.F. Briat, and C. Curie, Dual regulation of the Arabidopsis high-affinity root iron uptake system by local and long-distance signals. Plant Physiol, 2003. 132(2): p. 796-804.

37. Vert, G., J.F. Briat, and C. Curie, Arabidopsis IRT2 gene encodes a root-periphery iron transporter. Plant J, 2001. 26(2): p. 181-9.

38. Schmidt, W., Iron solutions: acquisition strategies and signaling pathways in plants. Trends Plant Sci, 2003. 8(4): p. 188-93.

39. Chaney, R.L., et al., Root hairs on chlorotic tomatoes are an effect of chlorosis rather than part of the adaptive Fe-stress-response. Journal of Plant Nutrition, 1992. 15(10): p. 1857-1875.

40. Fournier, J.M., E. Alcántara, and M.D. de la Guardia, Organic acid accumulation in roots of two sunflower lines with a different response to iron deficiency. Journal of Plant Nutrition, 1992. 15(10): p. 1747-1755.

41. Ma, J.F., Plant Root Responses to Three Abundant Soil Minerals: Silicon, Aluminum and Iron. Critical Reviews in Plant Sciences, 2005. 24(4): p. 267-281. 


\section{Chapter 5: Conclusions and Future Studies}

Three plant biological studies using radiotracer methods have been discussed in the previous chapters. This chapter aims to highlight the important findings in those studies as well as to discuss the direction of future work.

In Chapter 2, heavy metal accumulation in the opt3-2 mutant Arabidopsis was studied. We found that $\mathrm{Pb}^{2+}$ over-accumulates in opt3-2 mutant plants. Using radioactive

${ }^{203} \mathrm{~Pb}$ to further explore the distribution and uptake dynamics of $\mathrm{Pb}$ ions in the opt3-2 mutant, we found that the over-accumulation of $\mathrm{Pb}$ is not due to a kinetically rapid $\mathrm{Pb}$ uptake, but rather long-term accumulation. We also found that the up-regulated $\mathrm{Fe}$ transporter IRT1 in the roots of the opt3-2 mutant does not transport $\mathrm{Pb}^{2+}$. It is likely that other up-regulated transporters play a role in transporting $\mathrm{Pb}^{2+}$ in the opt3-2 mutant. Further experimentation will need to be conducted to identify the up-regulated transporters involved in $\mathrm{Pb}^{2+}$ trafficking. For example, the opt3-2/ transporter double mutants (which have knocked-out the Atopt3 gene and those transporter genes) should be grown under $\mathrm{Pb}$ treatment conditions. The plant growth of the double mutant plants would be compared to the opt3-2 mutant to identify if any of the transporters are able to transport $\mathrm{Pb}^{2+}$. Previous studies proposed that glutathione might play a key role in heavy metal trafficking throughout the plant (unpublished data from David G. MendozaCozatl). The glutathione level in the opt3-2 mutant might be elevated. We conducted ${ }^{35} \mathrm{~S}$ glutathione uptake assays (See Appendix A Fig. A1-8) in both the opt3-2 mutant and the WT plants and found that the opt3-2 mutant tended to distribute the ${ }^{35}$ S-glutathione to all leaves, while the WT plants clearly showed an asymmetric distribution of the ${ }^{35} \mathrm{~S}$ - 
glutathione in the leave. However more replicates of this experiment will need to be conducted in the future to verify the role of glutathione in heavy metal trafficking.

In Chapter 3, the carbon metabolism and translocation in Arabidopsis plants were investigated using both ${ }^{12} \mathrm{C}$ and ${ }^{11} \mathrm{C}$ methods. The ${ }^{11} \mathrm{CO}_{2}$ radiotracer, produced at $\mathrm{BNL}$, provided us the opportunity to explore the dynamics of carbon flux in plants, as well as diurnal effects on carbon flux. According to the conceptual model mentioned in Agtuca's study [1], the ${ }^{11} \mathrm{C}$ data demonstrates snapshots of the dynamics of carbon flux, and is combined with ${ }^{12} \mathrm{C}$ analysis, which demonstrates the carbon pool size (such as the sugar pool size), to gives a full picture of the fluctuation of carbon metabolism in both the starch mutants and the WT (Fig. 5-1).

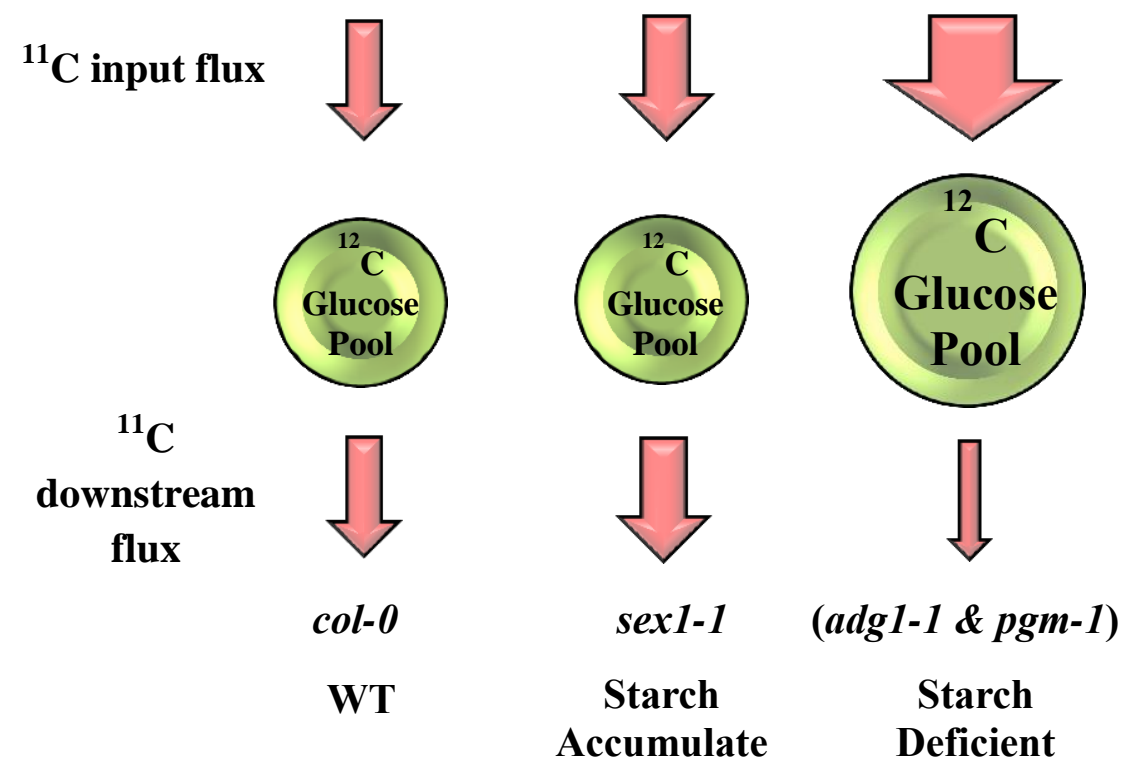

Figure 5-1. A conceptual model used to interpretate ${ }^{12} \mathrm{C}$ and ${ }^{11} \mathrm{C}$ data. 
In this study, we found that starch regulation is essential not only for plant growth, but also affects sugar metabolism (Fig. 5-1), carbon allocation, and root exudation. Previous research has shown that plants interact with the rhizosphere through root exudation, which affects the species of the micro-organisms living in the rhizosphere; in turn, the altered rhizosphere will influence the plant growth [2,3]. Our ${ }^{11} \mathrm{C}$ data showed that there were large amounts of carbon translocated to the belowground of the plants (which might be in forms of sugars or auxin), and secreted into the rhizosphere (especially with the starch deficient mutants). However, more experiments are needed to identify the species of the carbon compounds translocated to belowground and exuded into the rhizosphere. For example, an LC-MS method could potentially be developed in the future to analyze the ${ }^{11} \mathrm{C}$-labeled carbon compounds in plant roots $[4,5]$.

In Chapter 4, the relationship between Fe status and carbon metabolism was discussed. Two iron-transport mutants and the WT Arabidopsis were used for this study. In this study, we found that the Fe status in plants affects the carbon fixation ability in plant leaves, and also alters carbon partitioning (e.g., organic acid production), allocation, and root exudation in plants. Diurnal effects on carbon metabolism and allocation were observed in this study as well. Previous studies [6,7] have shown that, under Fe deficient conditions, plants tend to re-direct carbon to organic acid production in order to acquire more $\mathrm{Fe}$ from the rhizosphere through chelation. In our ${ }^{11} \mathrm{C}$ data, the irt1-1 mutant showed high levels of carbohydrates in the below-ground segments and root exudates under Fe deficient conditions. Thus, it is possible that most of the carbon compounds were in the form of organic acids. To validate this hypothesis, further experiments, including as LC-MS analyses, are needed [4, 5]. 


\section{References}

1. Agtuca, B., Rieger, E., Hilger, K., Song , L., Ferrieri, R.A., Carbon-11 used to Study the Physiological and Metabolic Basis for Hormonal Cross-Talk between Auxin and Salicylic Acid in Maize (Zea mays L.) Root Development. (in review), 2013.

2. Hirsch A.M., e.a., Molecular signals and receptors: controlling rhizosphere interactions between plants and other organisms. Ecology, 2003. 84: p. 858-68.

3. $\quad$ Bais, H.P., et. al., The Role of Root Exudates in Rhizosphere Interactions with Plants and Other Organisms. Annu Rev Plant Biol, 2006. 57: p. 233-66.

4. Chen, Z., et al., Confirmation and determination of carboxylic acids in root exudates using LC-ESI-MS. J Sep Sci, 2007. 30(15): p. 2440-6.

5. Oburger, E., et al., Evaluation of a novel tool for sampling root exudates from soil-grown plants compared to conventional techniques. Environmental and Experimental Botany, 2013. 87(0): p. 235-247.

6. Abadía, J., et al., Organic acids and Fe deficiency: a review. Plant and Soil, 2002. 241(1): p. 75-86.

7. De Vos, C.R., H.J. Lubberding, and H.F. Bienfait, Rhizosphere Acidification as a Response to Iron Deficiency in Bean Plants. Plant Physiology, 1986. 81(3): p. 842-846. 
Appendix A: Supplementary Data 


\section{A1. Chapter 2}

\section{A1.1 Heavy Metal Treatments}

Both the WT and the opt3-2 mutant plants were grown in media with different metal treatments (Table A1-1). The purpose of these experiments was to investigate if the opt3-2 mutant would show distinctive phenotypes under metal treatments compared to the WT plants. Plants were grown under the same growth conditions as described in the Plant Growth Conditions in Chapter 2.

Except under $\mathrm{Pb}, \mathrm{Hg}$ and $\mathrm{Cd}$ treatments, we did not see dramatic differences between the WT and the opt3-2 mutant plants when they were grown in the presence of the other metals or the various treatments.

Table A1-1. Heavy metal treatments tested on the opt3-2 mutant plants.

\begin{tabular}{|c|c|c|c|}
\hline Metal compounds & \multicolumn{3}{|c|}{ Concentration/ $\boldsymbol{\mu M}$} \\
\hline $\mathrm{CdCl}_{2}$ & 10 & 20 & 30 \\
\hline $\mathrm{HgCl}_{2}$ & 5 & 10 & 20 \\
\hline $\mathrm{Pb}(\text { acetate })_{2}$ & 250 & 500 & 750 \\
\hline $\mathrm{UO}_{2}(\text { acetate })_{2}$ & 250 & 500 & $600 / 750$ \\
\hline $\mathrm{Na}_{2} \mathrm{HAsO}_{4}$ & 30 & 60 & 120 \\
\hline $\mathrm{FeCl}_{3}$ & 50 & 100 & 200 \\
\hline $\mathrm{GaCl}_{3}$ & 50 & 100 & $200 / 250$ \\
\hline
\end{tabular}




\section{A1.2 Seed Germination and Plant Root Growth under Different Heavy Metal Treatments}

For seed germination measurement, seeds were surface sterilized and cold-treated as described in the Plant Growth Condition section in Chapter 2, before germination in the MS agar media containing different concentrations of metals. The number of germinated seeds was manually counted under a microscope during the first three days. The germination percentage was calculated as shown in the equation below and in Figure A1-1.

$$
\text { Germination } \%=\frac{\# \text { of germinated seeds }}{\text { Total } \# \text { of seeds }} \times 100 \%
$$

For root growth measurements, seeds were surface sterilized and cold-treated as described in the Plant Growth Condition section in Chapter 2, before germination in halfstrength MS agar media. One-week-old seedlings were then transferred to agar media containing different concentrations of the metals. After 1-1.5 weeks of a growing period, pictures were taken using a camera and the root length was measured using the ImageJ software. The percentage of root elongation inhibition (compared to the WT under no metal treatment) was calculated as shown in the equation below and shown in Figure A12:

$$
\text { Root elongation inhibition } \%=\frac{\left|\mathrm{L}_{\text {metal }}-\mathrm{L}_{\text {control }}\right|}{\mathrm{L}_{\text {control }}} \times 100 \%
$$

$\mathrm{L}_{\text {metal }}$ : root length of the WT or the opt3-2 mutant plants grown under metal treatments. $\mathrm{L}_{\text {control }}$ : root length of the WT or the opt3-2 mutant plants grown without metal treatments. 

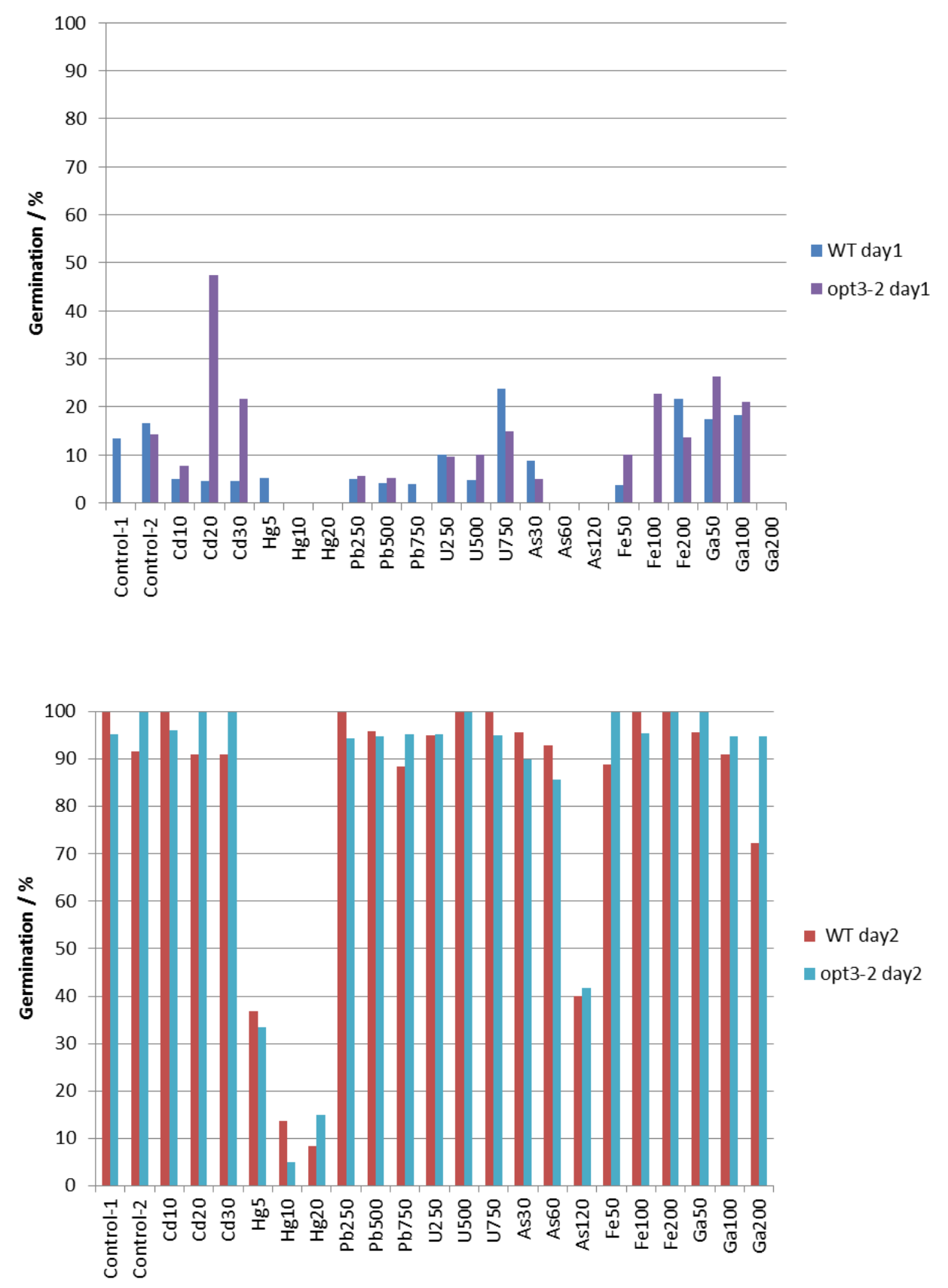

(Continued to the next page) 


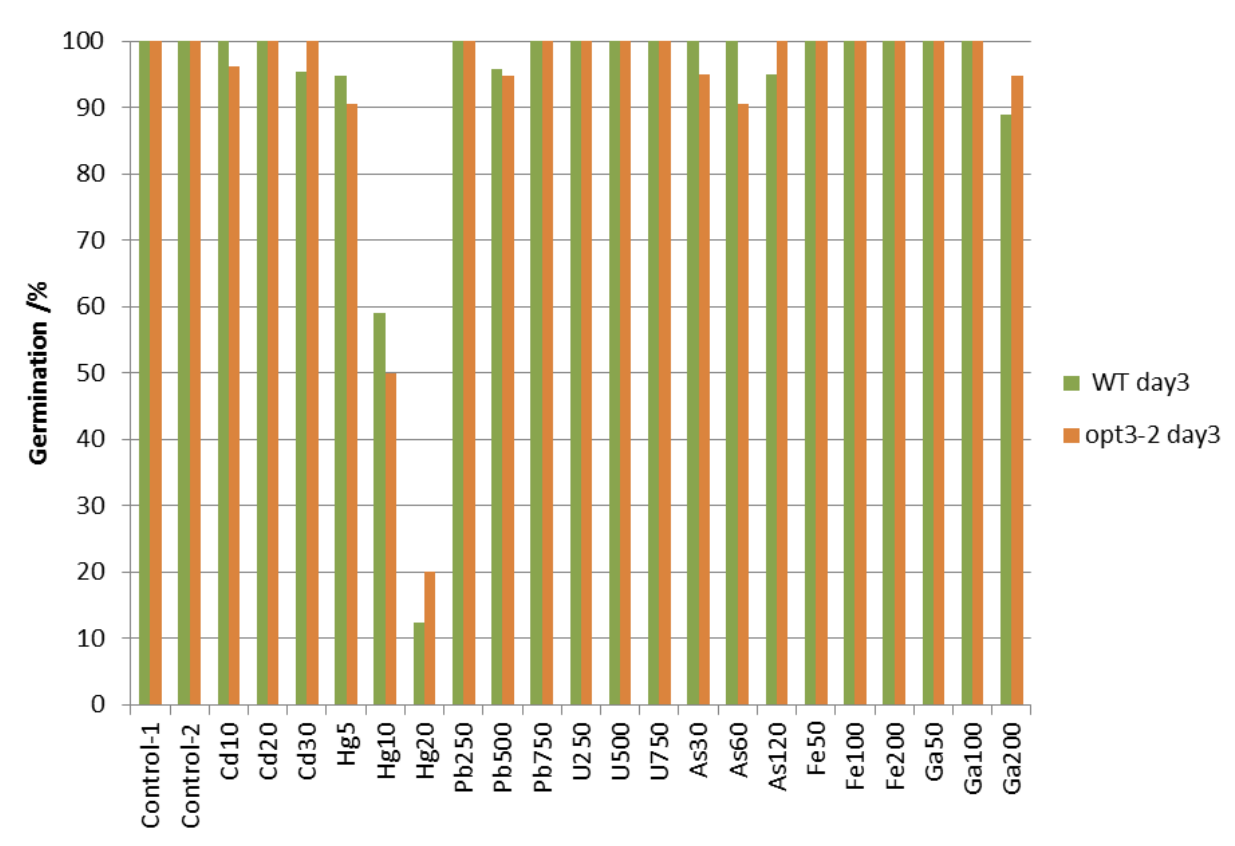

Figure A1-1. Comparisons of seed germination rates under different heavy metal treatments within the first three days. The number after the each heavy metal symbol represents the concentration in $\mu \mathrm{M}$ of that heavy metal. $\mathrm{n}=1$.

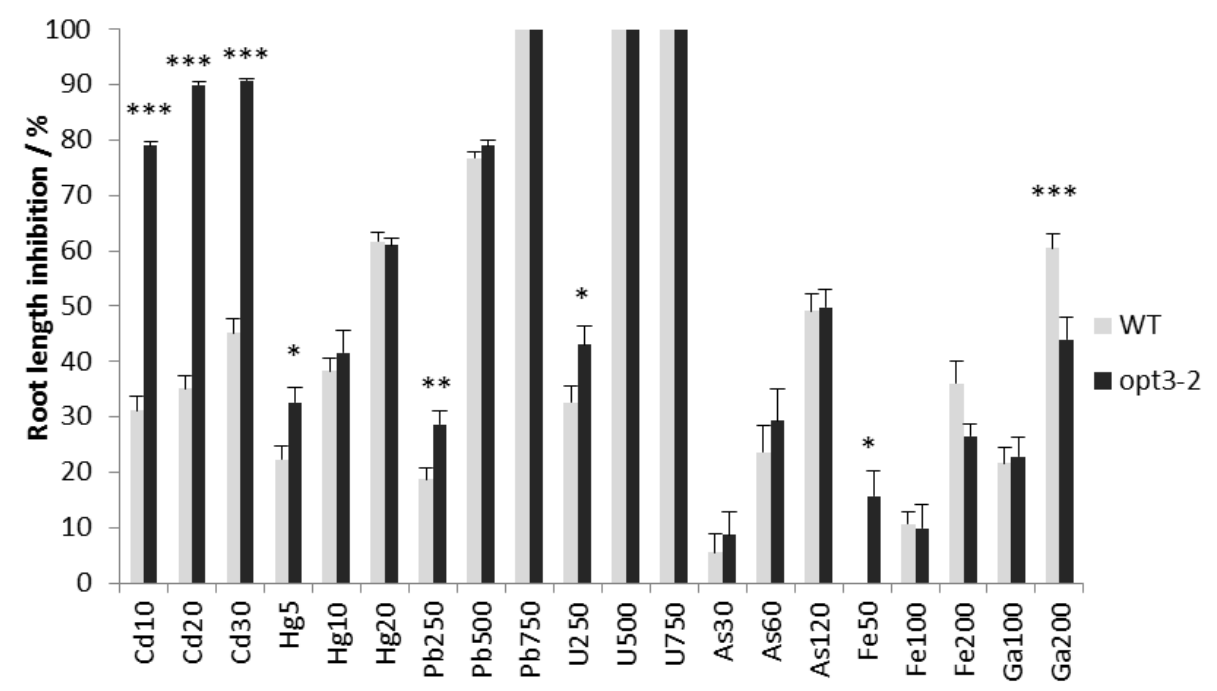

Figure A1-2. Comparisons of root elongation under different heavy metal treatments. The number after the each heavy metal symbol represents the concentration in $\mu \mathrm{M}$ of that heavy metal. $\mathrm{n}=8-12$ seedlings, mean $\pm \mathrm{SE}$. *, $\mathrm{p}<0.05$; $* *, \mathrm{p}<0.01 ; * * *, \mathrm{p}<0.001$. 


\section{A1.3 Characterization of RBS}

We have tried to use fluorescence imaging technology to investigate the distribution of $\mathrm{Hg}^{2+}$ in plant tissues. Previous studies have shown that Rhodamine B Thiolactone (RBS, Scheme A1-1) is a promising $\mathrm{Hg}^{2+}$ fluorescence probe, which has enhanced fluorescence at around $585 \mathrm{~nm}\left(\lambda_{\mathrm{ex}}=530 \mathrm{~nm}\right)$ when interacting with $\mathrm{Hg}^{2+}$. RBS was shown to be selectivefor $\mathrm{Hg}^{2+}$ detection in the presence of other metal ions commonly seen in plant tissues (Scheme A1-2) [1]. RBS was synthesized based on Wen Shi's method [2] and characterized using both ${ }^{1}$ H-NMR (Fig. A1-3) and ${ }^{13}$ C-NMR (Fig. A1-4). Before testing on plant tissues, the RBS solution was evaluated with several concentrations of $\mathrm{HgCl}_{2}$ solutions (Fig. A1-5).

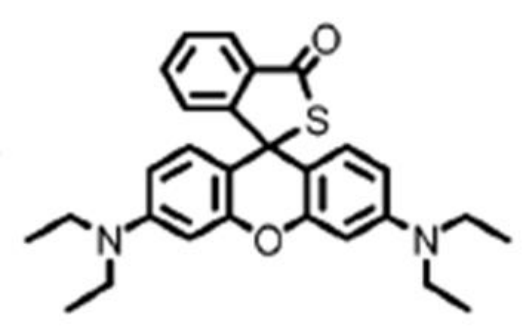

Scheme A1-1. Structure of RBS. 

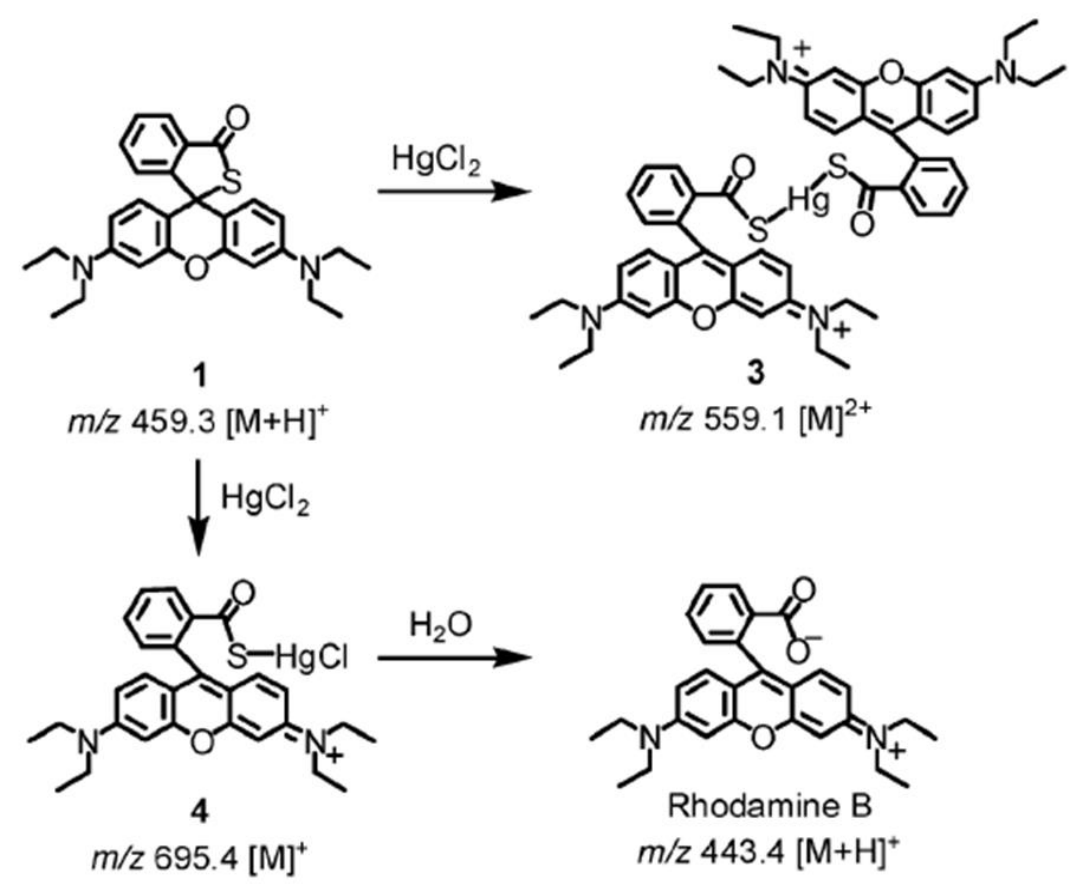

Scheme A1-2. Possible reaction mechanisms of RBS with $\mathrm{HgCl}_{2}$. 


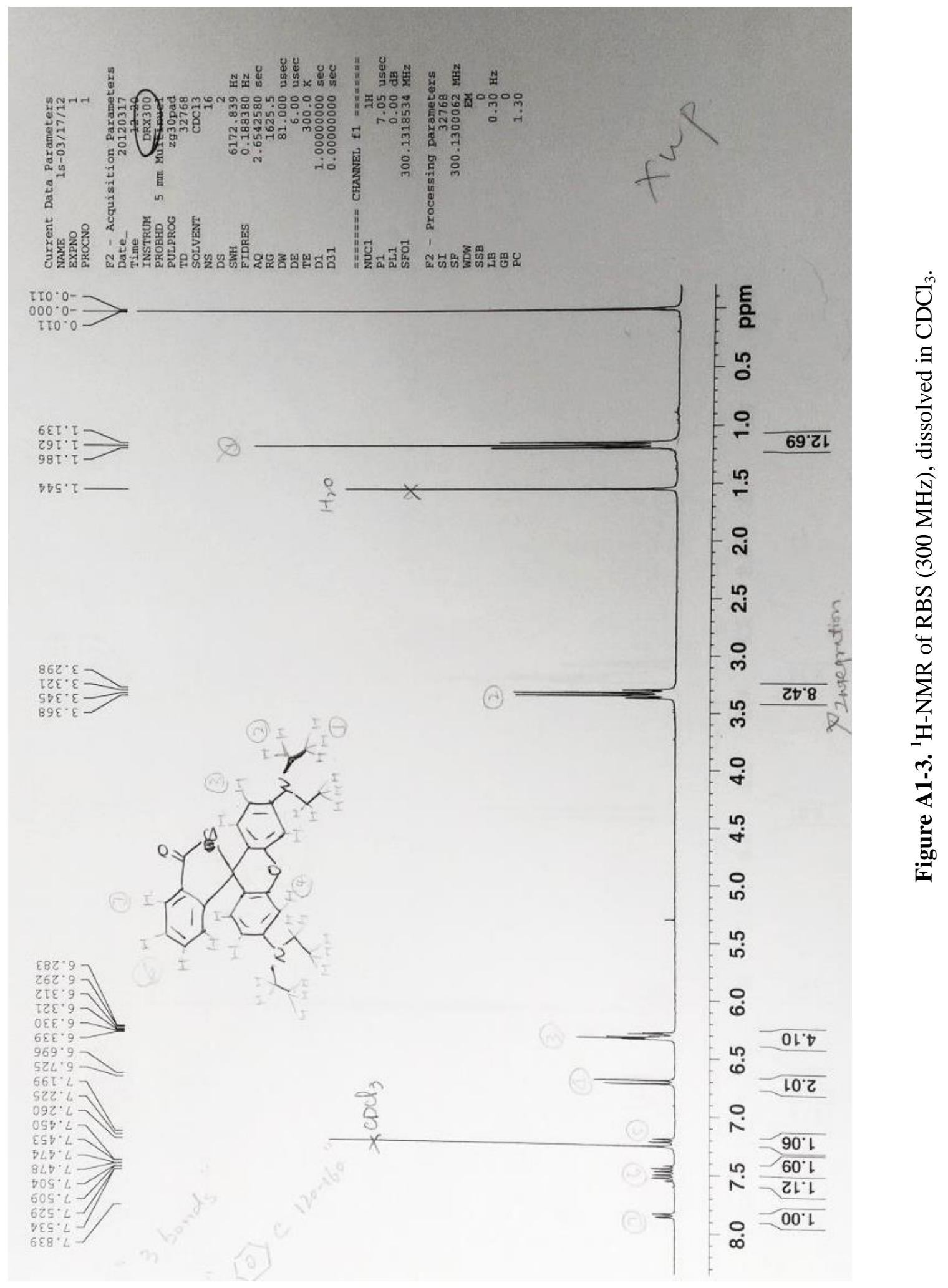




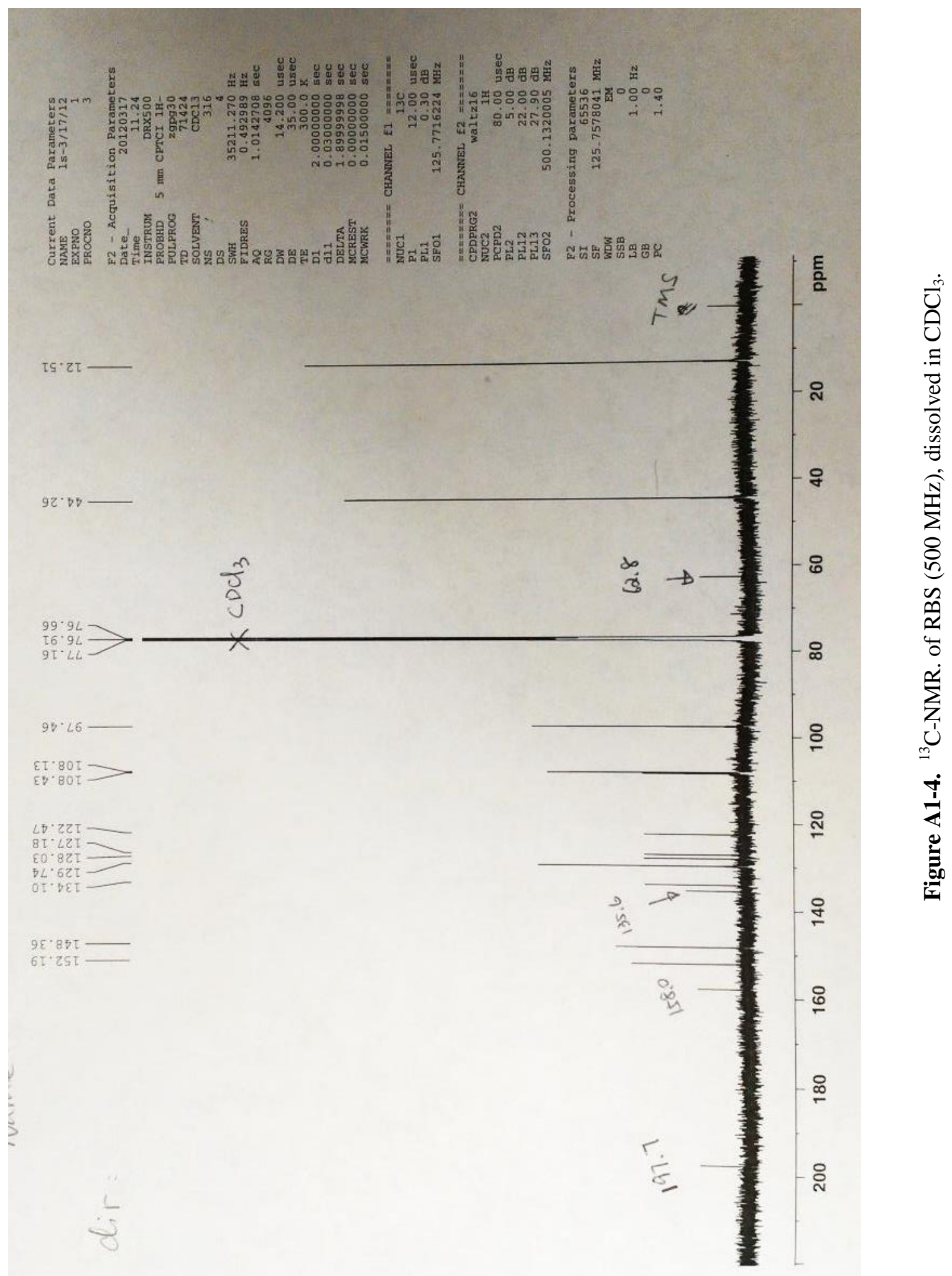




\section{Appendix A 113}

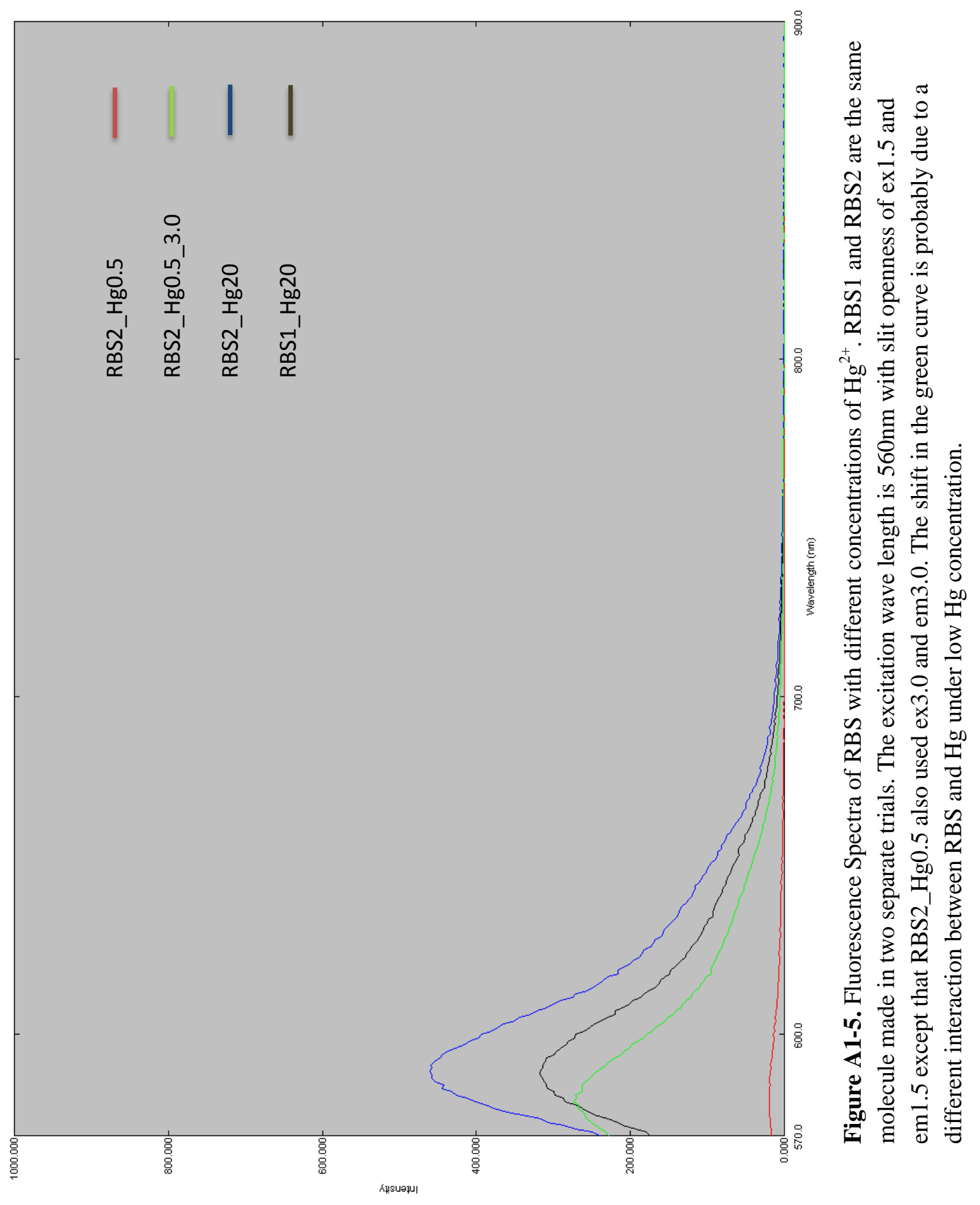


A1.4 Plant physiology (including opt3-2 mutant, irt1-1 mutant and wild type)

The irt1-1 mutant was added into this experiment to make a comparison with the opt3-2 mutant and the wild type Arabidopsis. Plants were grown using the same method described in Plant Growth Conditions in Chapter 2. The only difference is that an extra $50 \mu \mathrm{M}$ of $\mathrm{Fe}^{3+}$ was added into the media to keep the normal growth of the irt1-1 mutant.

The results showed that the growth of the irt -1 mutant was inhibited under high concentrations of $\mathrm{Pb} / \mathrm{Hg}$ treatment, which is at a similar level to that observed with the opt3-2 mutant. This result indicates that IRT1-1 does not play a key role in $\mathrm{Pb} / \mathrm{Hg}$ trafficking.
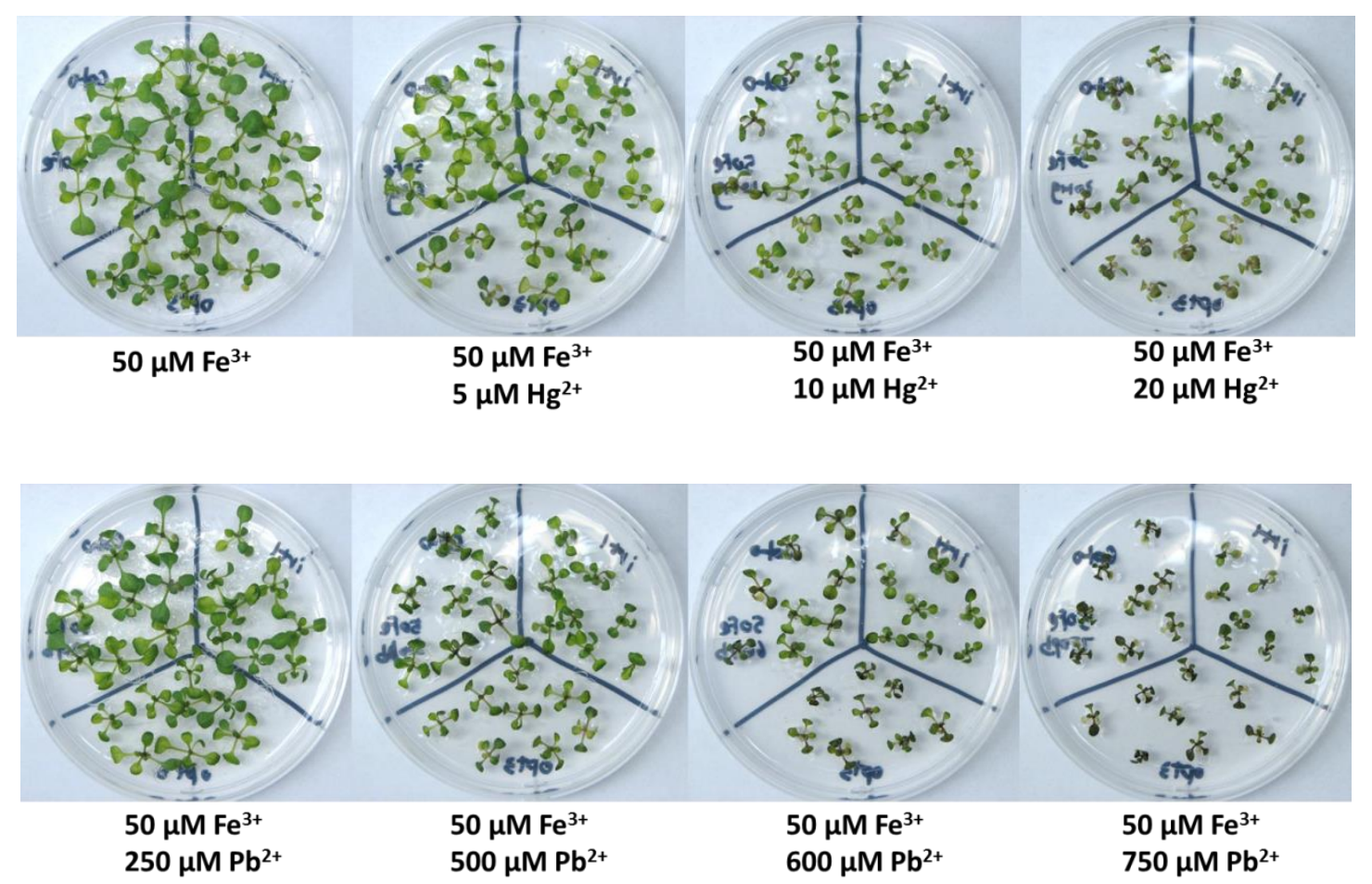

Figure A1-6. Comparison of shoot growth of the opt3-2, irt1-1 mutants and the wild type Arabidopsis with various concentrations of $\mathrm{Pb} / \mathrm{Hg}$ treatments. $50 \mu \mathrm{M} \mathrm{Fe}^{3+}$ was added to all the media to ensure a healthy growth of the irt $1-1$ mutant. 

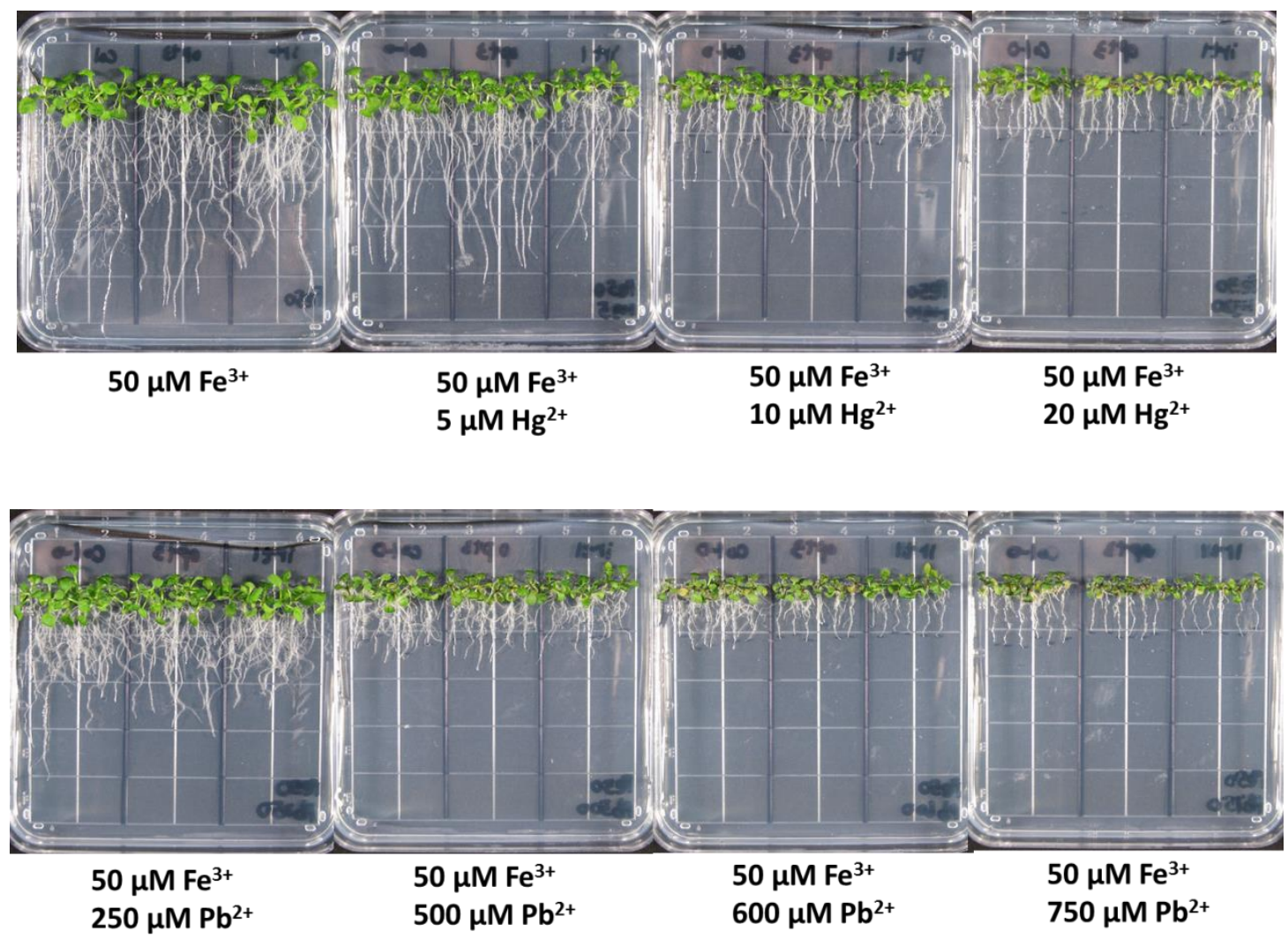

Figure A1-7. Comparison of the root growth for the opt3-2, irt1-1 mutants and the wild type Arabidopsis under various concentrations of $\mathrm{Pb} / \mathrm{Hg}$ treatments. $50 \mu \mathrm{M} \mathrm{Fe}^{3+}$ was added in all the media to ensure a healthy growth of the irt $1-1$ mutant. 


\section{A1.5 Glutathione uptake and distribution in the opt3-2 mutant}

In this experiment, one-month old WT and opt3-2 mutant plants were used (grown in soil). A load leaf was first abraded with a fabric wheel to generate holes on the leaf surface so that the incubation solution (Table A1-2) can be taken up through the load leaf. Then the load leaf was placed into a manually cut $1.5 \mathrm{ml}$ centrifuge tube (Fig. A1-8). After 24 hours of incubation, the load leaf was removed from the plant. The entire shoot was then removed from the roots and dissected into individual leaves (Fig. A1-9). The leaves were then exposed to the phosphor screen for 10 hours to acquire the phosphor images. For solution preparation and experimental parameters, please refer to Table A1-2.

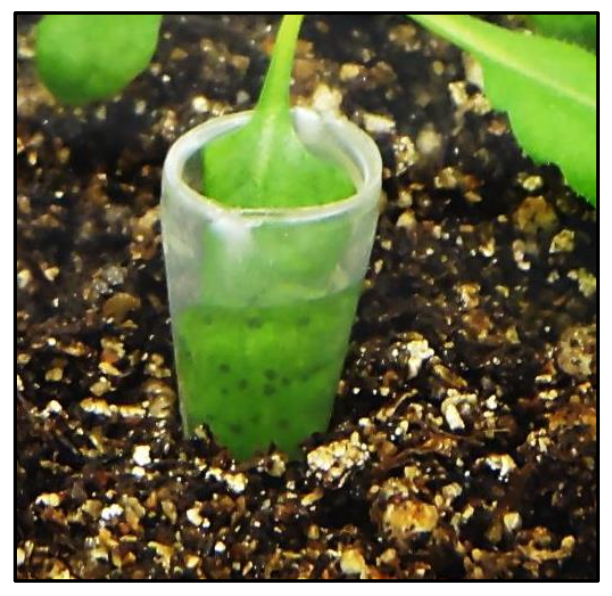

Figure A1-8. Illustration of load leaf incubation in the $\left[{ }^{35} \mathrm{~S}\right]$-glutathione containing solution. 


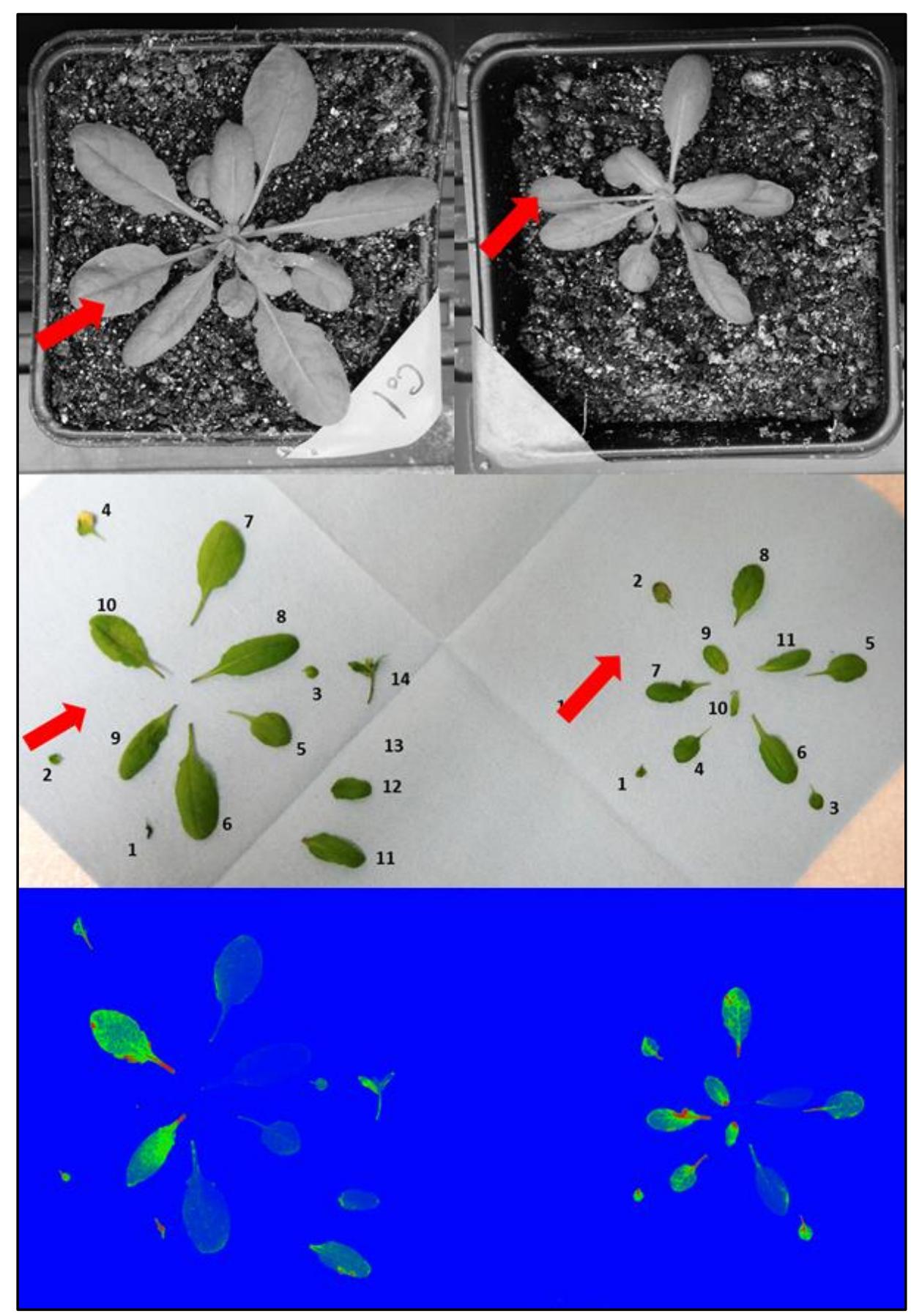

Figure A1-9. ${ }^{35}$ S-glutathione uptake through leaf administration and distribution patterns in the opt3-2 mutant (right) and the WT (left). Red arrows indicate the administration leaf, which are removed in the middle and the bottom pictures. 
Appendix A 118

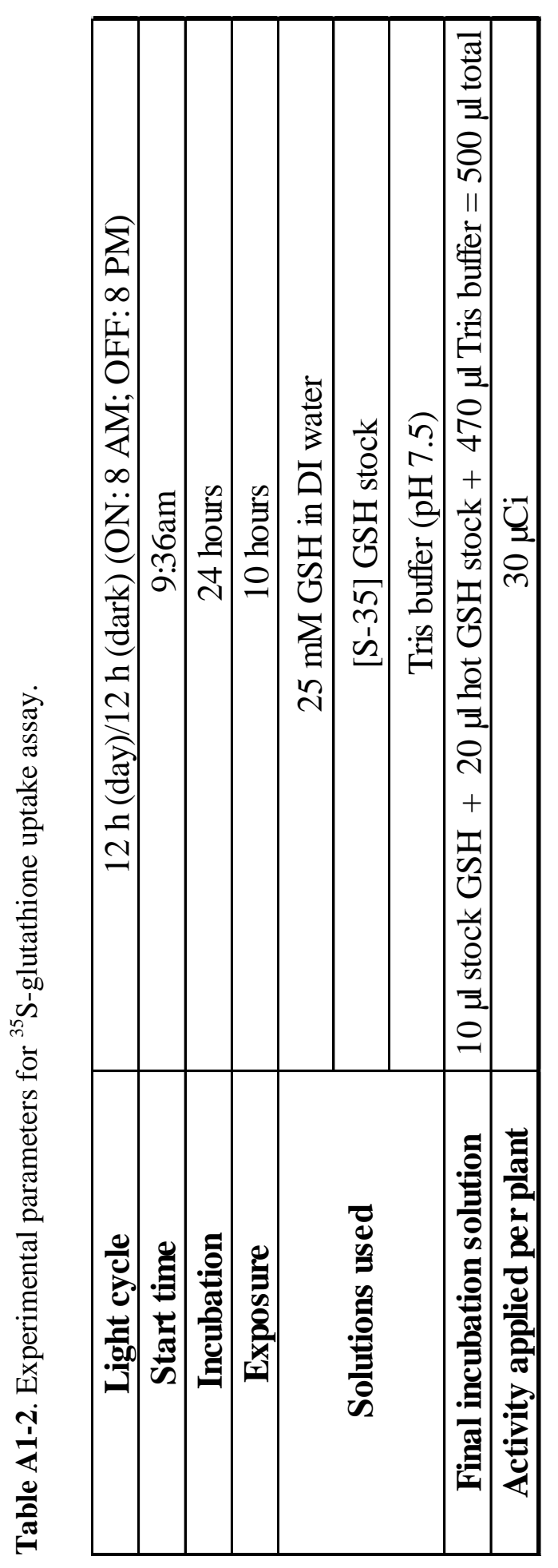


Table A1-3. Data of root / shoot growth of plants under $\mathrm{Hg}$ or $\mathrm{Pb}$ treatments.

\begin{tabular}{|c|c|c|c|c|}
\hline \multirow[b]{2}{*}{ Treatments } & \multicolumn{2}{|c|}{$\underline{W T}$} & \multicolumn{2}{|c|}{ opt3-2 } \\
\hline & $\begin{array}{l}\text { Shoots } \\
\text { (mg) }\end{array}$ & $\begin{array}{c}\text { Roots } \\
\text { (mg) }\end{array}$ & $\begin{array}{c}\text { Shoots } \\
\text { (mg) }\end{array}$ & $\begin{array}{c}\text { Roots } \\
\text { (mg) }\end{array}$ \\
\hline \multirow{4}{*}{ Control } & 22.43 & 8.37 & 15.50 & 8.13 \\
\hline & 21.93 & 8.67 & 16.57 & 10.77 \\
\hline & 25.37 & 9.13 & 13.40 & 8.67 \\
\hline & 20.80 & 9.07 & 14.80 & 8.60 \\
\hline \multirow{2}{*}{$\begin{array}{c}\text { avg. } \\
\text { SE }\end{array}$} & 22.63 & 8.81 & 15.07 & 9.04 \\
\hline & 0.10 & 0.02 & 0.07 & 0.06 \\
\hline \multirow{4}{*}{ Hg10 } & 17.63 & 6.47 & 14.20 & 5.50 \\
\hline & 20.43 & 8.33 & 10.00 & 3.73 \\
\hline & 20.00 & 7.93 & 11.20 & 4.53 \\
\hline & 15.33 & 5.23 & 13.17 & 5.57 \\
\hline \multirow{2}{*}{$\begin{array}{c}\text { avg. } \\
\text { SE }\end{array}$} & 18.35 & 6.99 & 12.14 & 4.83 \\
\hline & 0.12 & 0.07 & 0.09 & 0.04 \\
\hline \multirow{4}{*}{ Hg20 } & 16.50 & 5.40 & 8.57 & 3.20 \\
\hline & 14.53 & 5.07 & 10.73 & 2.87 \\
\hline & 13.93 & 4.20 & 8.33 & 2.27 \\
\hline & 11.73 & 4.80 & 9.60 & 2.77 \\
\hline \multirow{2}{*}{$\begin{array}{c}\text { avg. } \\
\text { SE }\end{array}$} & 14.18 & 4.87 & 9.31 & 2.78 \\
\hline & 0.10 & 0.03 & 0.05 & 0.02 \\
\hline \multirow{4}{*}{ Pb500 } & 14.40 & 5.53 & 10.20 & 3.83 \\
\hline & 14.90 & 7.10 & 11.33 & 4.17 \\
\hline & 17.47 & 7.97 & 11.23 & 2.77 \\
\hline & 14.33 & 4.77 & 10.20 & 3.17 \\
\hline \multirow{2}{*}{$\begin{array}{c}\text { avg. } \\
\text { SE }\end{array}$} & 15.28 & 6.34 & 10.74 & 3.48 \\
\hline & 0.07 & 0.07 & 0.03 & 0.03 \\
\hline \multirow{4}{*}{ Pb750 } & 8.80 & 1.97 & 6.73 & 0.77 \\
\hline & 8.47 & 1.90 & 6.67 & 1.23 \\
\hline & 10.03 & 2.13 & 5.80 & 0.77 \\
\hline & 10.37 & 2.53 & 5.83 & 0.97 \\
\hline avg. & 9.42 & 2.13 & 6.26 & 0.93 \\
\hline SE & 0.05 & 0.01 & 0.03 & 0.01 \\
\hline
\end{tabular}


Table A1-4. Data of chlorophyll content

\begin{tabular}{|c|c|c|}
\hline \multirow{2}{*}{ Treatments } & \multicolumn{2}{|c|}{$\mathrm{Chl}(\mu \mathrm{g} / \mathrm{g}$ f.w.) } \\
\hline & $W T$ & opt3-2 \\
\hline \multirow{5}{*}{ Control } & 607 & 626 \\
\hline & 609 & 571 \\
\hline & 690 & 545 \\
\hline & 755 & 776 \\
\hline & & 569 \\
\hline avg. & 665 & 617 \\
\hline SE & 36 & 42 \\
\hline \multirow{5}{*}{ Hg10 } & 581 & 667 \\
\hline & 809 & 508 \\
\hline & 927 & 659 \\
\hline & 846 & 342 \\
\hline & 577 & 482 \\
\hline avg. & 748 & 531 \\
\hline SE & 72 & 61 \\
\hline \multirow{6}{*}{ Hg20 } & 412 & 463 \\
\hline & 635 & 400 \\
\hline & 556 & 411 \\
\hline & 639 & 400 \\
\hline & 470 & 305 \\
\hline & & 374 \\
\hline avg. & 542 & 392 \\
\hline SE & 45 & 21 \\
\hline \multirow{5}{*}{ Pb500 } & 670 & 587 \\
\hline & 698 & 512 \\
\hline & 567 & 506 \\
\hline & 544 & 719 \\
\hline & 606 & \\
\hline avg. & 617 & 581 \\
\hline SE & 30 & 49 \\
\hline \multirow{5}{*}{$\mathrm{Pb} 750$} & 431 & 299 \\
\hline & 430 & 380 \\
\hline & 524 & 224 \\
\hline & 544 & 405 \\
\hline & 405 & \\
\hline avg. & 467 & 327 \\
\hline SE & 28 & 41 \\
\hline
\end{tabular}




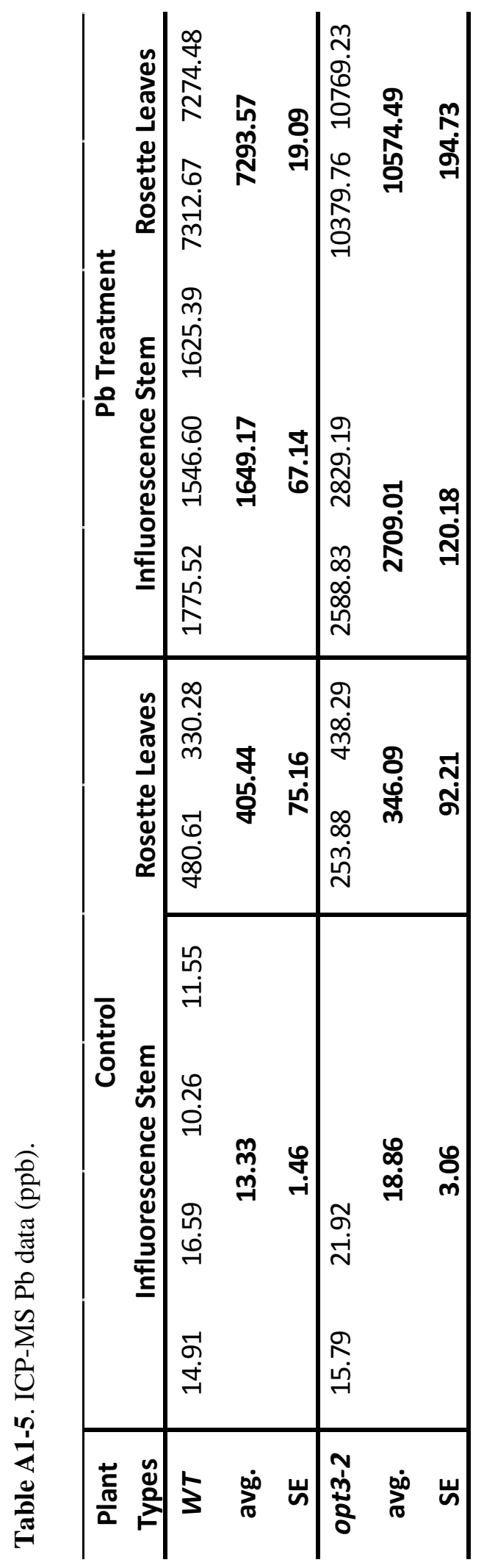




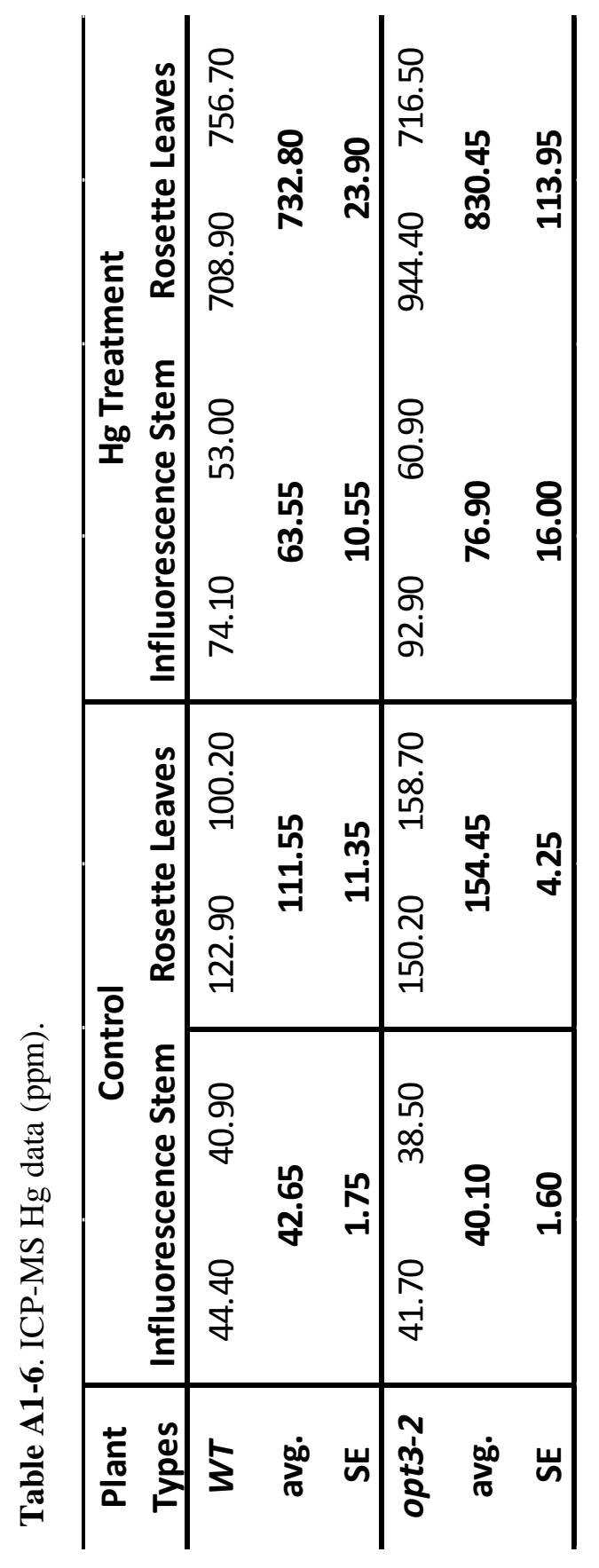


Table A1-7. ${ }^{203} \mathrm{~Pb}$ distribution in opt $3-2$ mutant and WT plants.

\begin{tabular}{|c|c|c|c|c|}
\hline $\begin{array}{l}\text { Date of } \\
\text { Expt. }\end{array}$ & $\begin{array}{l}\text { Tissue } \\
\text { Type }\end{array}$ & $\begin{array}{l}\text { Normalized Activity } \\
\text { (cpm/g f.w.) }\end{array}$ & $\begin{array}{c}\text { Percentage of }{ }^{203} \mathrm{~Pb} \\
\text { transported to } \\
\text { shoots/roots }\end{array}$ & $\begin{array}{c}\text { Total }{ }^{203} \mathrm{~Pb} \\
\text { Activity } \\
\text { (entire plant, } \\
\text { cpm/g f.w.) }\end{array}$ \\
\hline $4 / 25 / 2013$ & $\begin{array}{l}\text { As } \\
\mathrm{Ar} \\
\mathrm{Bs} \\
\mathrm{Br}\end{array}$ & $\begin{array}{c}55765 \\
20443988 \\
7156 \\
24856210\end{array}$ & $\begin{array}{c}0.27 \\
99.73 \\
0.03 \\
99.97\end{array}$ & $\begin{array}{l}20499753 \\
24863366\end{array}$ \\
\hline $4 / 26 / 2013$ & $\begin{array}{l}\text { As } \\
\mathrm{Ar} \\
\mathrm{Bs} \\
\mathrm{Br}\end{array}$ & $\begin{array}{c}39861 \\
19817720 \\
49951 \\
14045935\end{array}$ & $\begin{array}{c}0.20 \\
99.80 \\
0.35 \\
99.65\end{array}$ & $\begin{array}{l}19857581 \\
14095886\end{array}$ \\
\hline 4/27/2013 & $\begin{array}{l}\mathrm{As} \\
\mathrm{Ar} \\
\mathrm{Bs} \\
\mathrm{Br}\end{array}$ & $\begin{array}{c}39578 \\
19660563 \\
22788 \\
17682114\end{array}$ & $\begin{array}{c}0.20 \\
99.80 \\
0.13 \\
99.87\end{array}$ & $\begin{array}{l}19700141 \\
17704902\end{array}$ \\
\hline $4 / 28 / 2013$ & $\begin{array}{l}\mathrm{As} \\
\mathrm{Ar} \\
\mathrm{Bs} \\
\mathrm{Br}\end{array}$ & $\begin{array}{c}45944 \\
39931622 \\
20454 \\
14561445\end{array}$ & $\begin{array}{c}0.11 \\
99.89 \\
0.14 \\
99.86\end{array}$ & $\begin{array}{l}39977566 \\
14581899\end{array}$ \\
\hline
\end{tabular}

Notes: As, WT shoots; Ar, WT roots; Bs, opt3-2 shoots; Br, opt3-2 roots. 


\section{A2. Chanpter 3}

Table A2-1. List of sugars

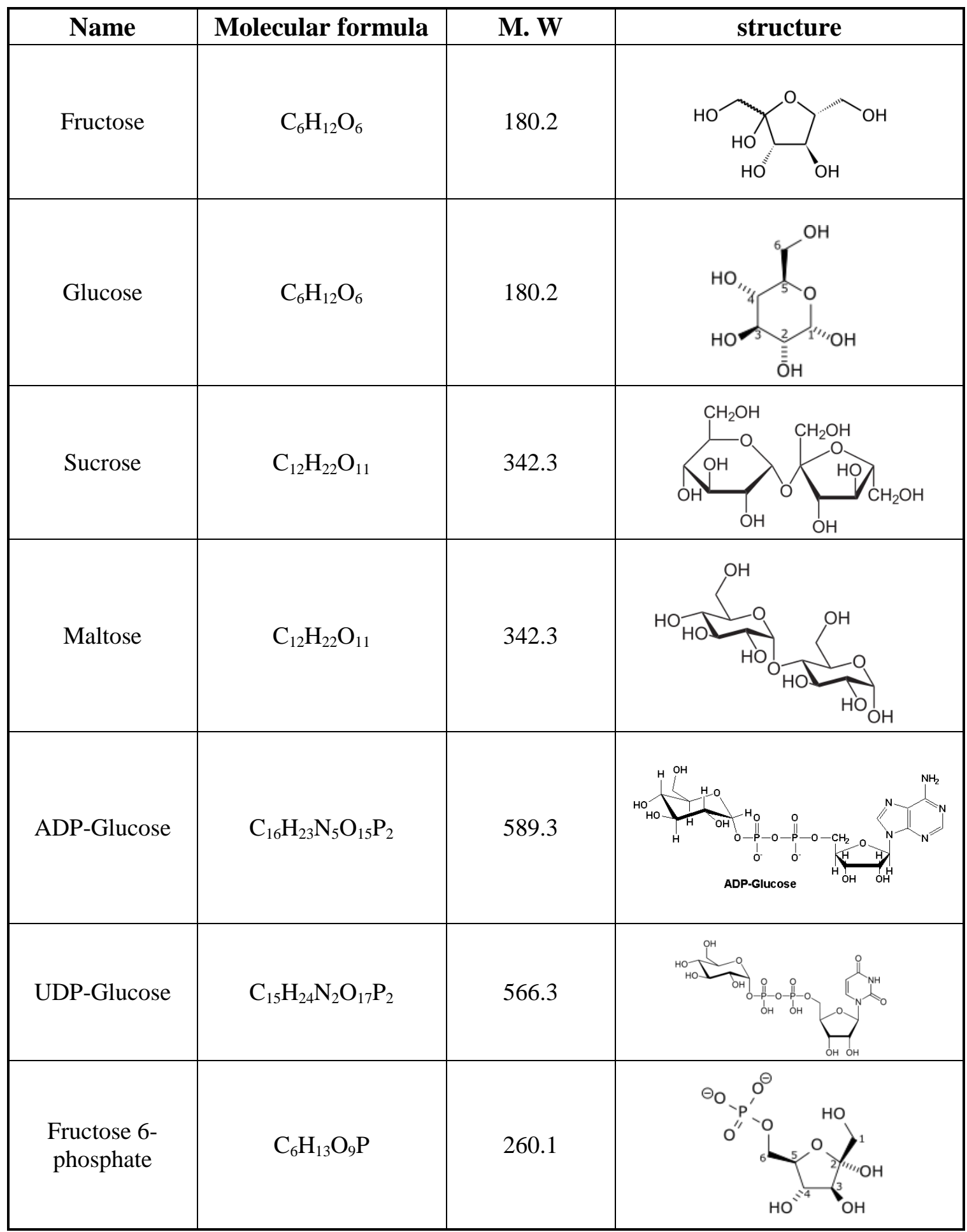




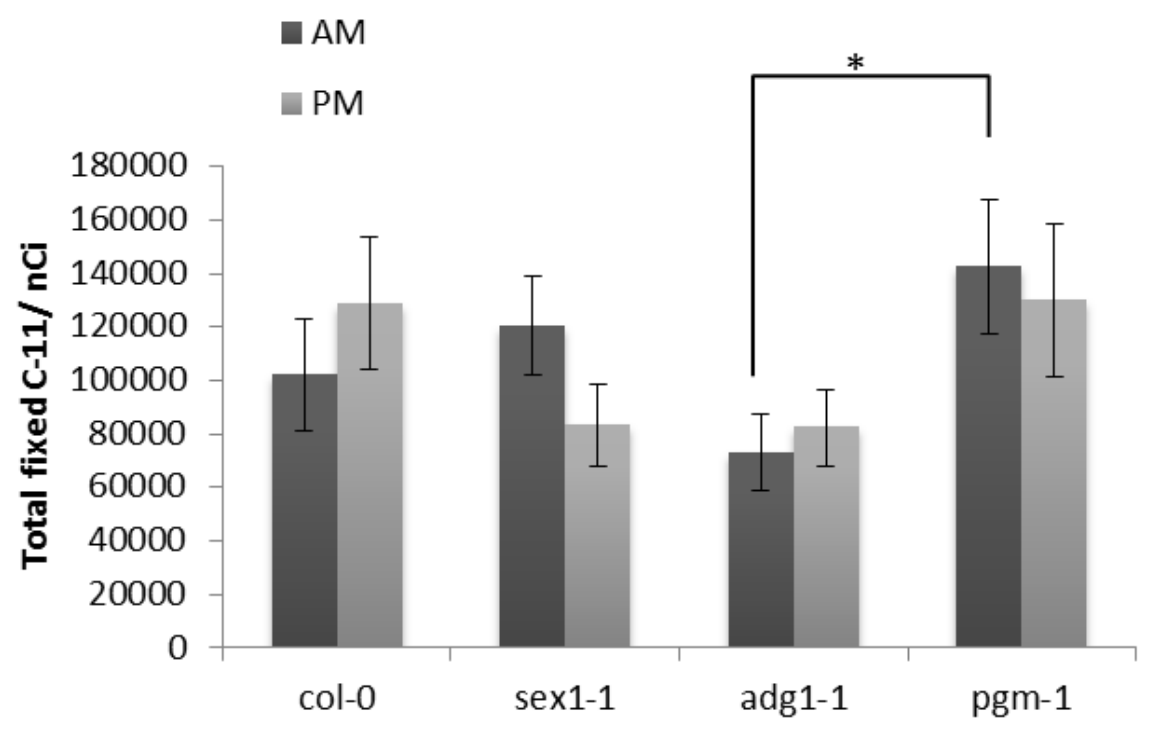

Figure A2-1. Carbon fixation in the three starch mutants and the wild type Arabidopsis. $\mathrm{n}=7-14$, mean \pm SE. *, $\mathrm{p} \leq 0.05 ;{ }^{* *}, \mathrm{p} \leq 0.01 ; * * *, \mathrm{p} \leq 0.001$.

Table A2-2. Data of Chlorophyll content.

\begin{tabular}{|c|c|c|c|c|}
\hline & \multicolumn{4}{|c|}{ Chlorophyll Content ( $\mu$ g/g f.w.) } \\
\hline & $W T$ & $\operatorname{sex} 1-1$ & adg1-1 & pgm-1 \\
\hline \multirow{10}{*}{ Replicates } & 504 & 488 & 321 & 408 \\
\hline & 485 & 326 & 413 & 600 \\
\hline & 466 & 512 & 398 & 479 \\
\hline & 539 & 469 & 574 & 522 \\
\hline & 568 & 482 & 334 & 531 \\
\hline & 480 & 466 & 347 & 550 \\
\hline & 532 & 574 & 432 & 599 \\
\hline & 450 & 334 & 383 & 504 \\
\hline & 481 & 419 & 516 & 611 \\
\hline & 341 & 418 & 523 & 593 \\
\hline avg. & 485 & 449 & 424 & 540 \\
\hline SE & 20 & 24 & 27 & 20 \\
\hline
\end{tabular}




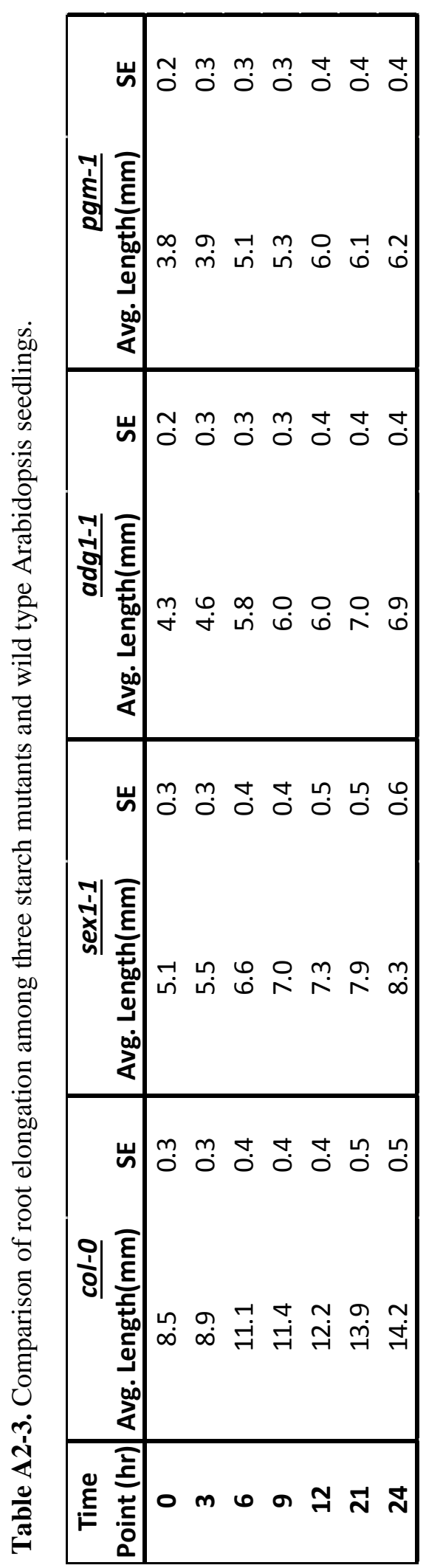




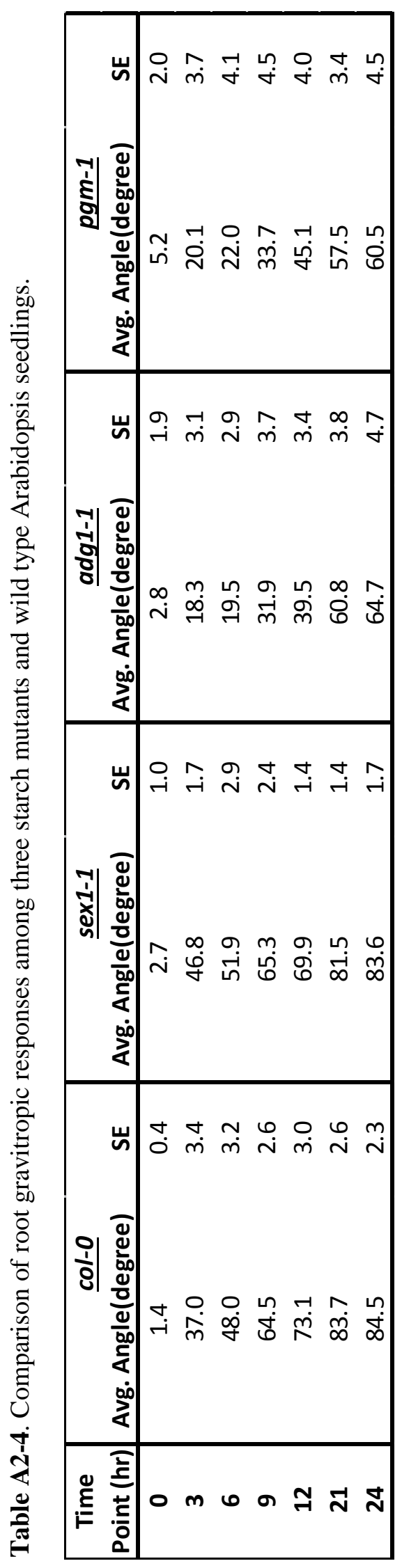




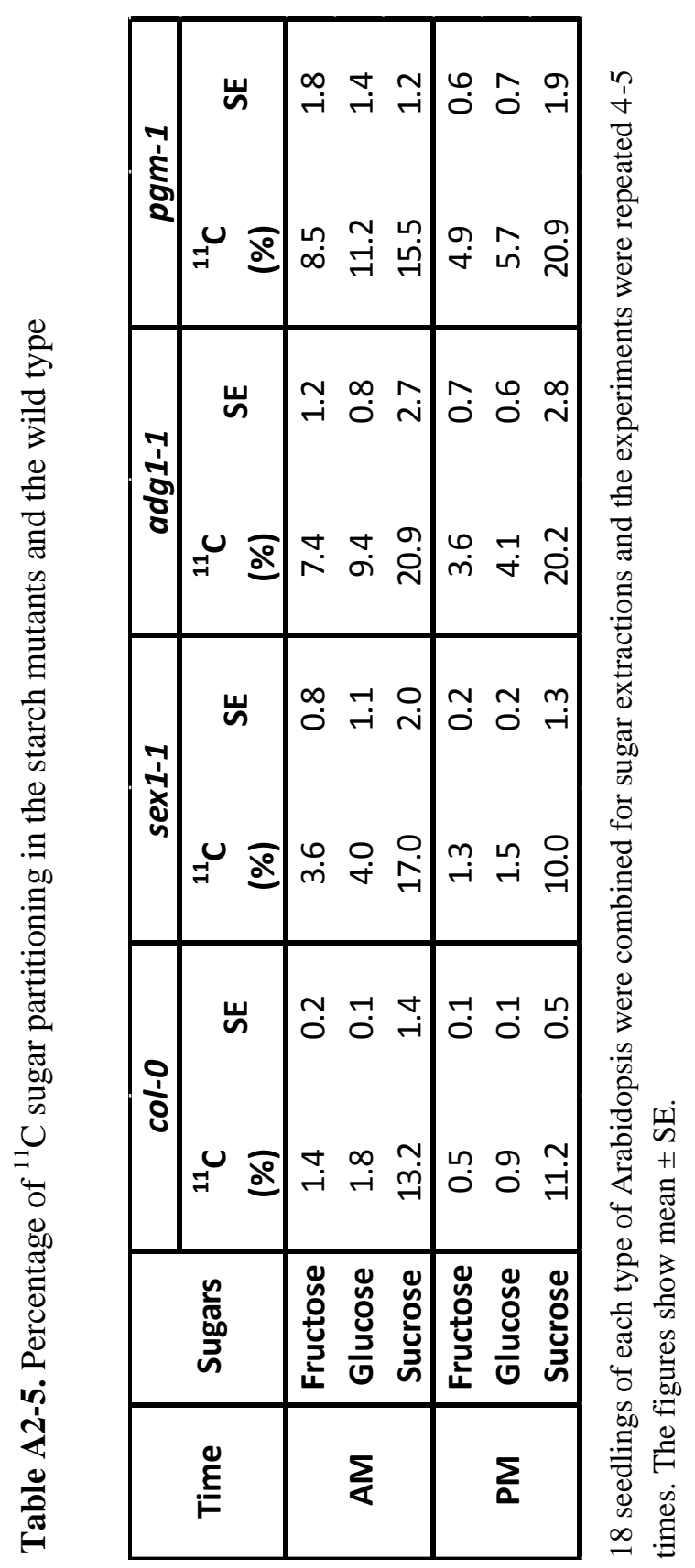




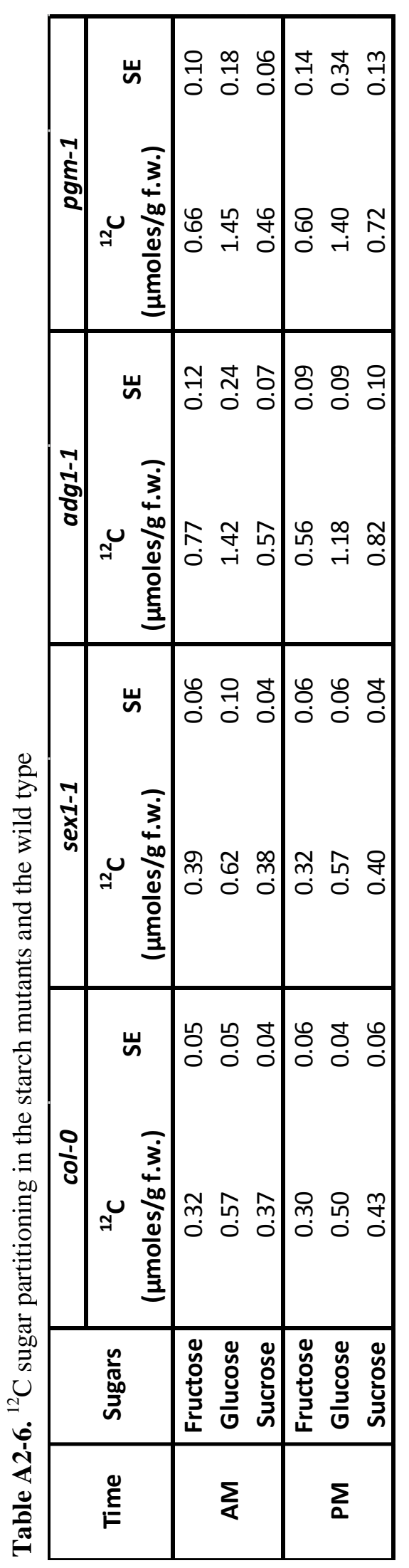


Table A2-7. Percentage of total fixed ${ }^{11} \mathrm{C}$ assimilated into insoluble carbohydrates.

\begin{tabular}{|c|c|cc|cc|}
\hline \multirow{2}{*}{ Plants } & \multirow{2}{*}{ Carbohydrates } & \multicolumn{2}{|c|}{ AM } & \multicolumn{2}{c|}{ PM } \\
\cline { 3 - 6 } & & $\begin{array}{l}\text { Fraction } \\
\text { of }{ }^{11} \mathbf{C}\end{array}$ & SE & \multicolumn{2}{|c|}{$\begin{array}{c}\text { Fraction } \\
\text { of }{ }^{11} \text { C }\end{array}$} \\
\hline \multirow{2}{*}{ WT } & Insoluble & 0.53 & 0.04 & 0.57 & 0.02 \\
& Extract & 0.47 & 0.04 & 0.43 & 0.02 \\
\hline \multirow{2}{*}{ sex1-1 } & Insoluble & 0.44 & 0.03 & 0.53 & 0.05 \\
& Extract & 0.56 & 0.03 & 0.47 & 0.05 \\
\hline \multirow{2}{*}{ adg1-1 } & Insoluble & 0.36 & 0.02 & 0.44 & 0.05 \\
& Extract & 0.64 & 0.02 & 0.56 & 0.05 \\
\hline \multirow{2}{*}{ pgm-1 } & Insoluble & 0.34 & 0.07 & 0.41 & 0.01 \\
& Extract & 0.66 & 0.07 & 0.59 & 0.01 \\
\hline
\end{tabular}

Table A2-8. ${ }^{11} \mathrm{C}$-carbohydrate relocation to belowground and root exudates.

\begin{tabular}{|c|cc|cc|}
\hline \multirow{4}{*}{ Plants } & \multicolumn{2}{|c|}{ AM } & \multicolumn{2}{|c|}{ PM } \\
\cline { 2 - 5 } & $\begin{array}{c}{ }^{11} \text { C transported to } \\
\text { belowground (\%) }\end{array}$ & SE & $\begin{array}{c}{ }^{11} \text { C transported } \\
\text { to } \\
\text { belowground (\%) }\end{array}$ & SE \\
\hline $\boldsymbol{W T}$ & 9.9 & 1.0 & 12.4 & 0.5 \\
sex1-1 & 13.2 & 1.0 & 10.2 & 0.6 \\
adg1-1 & 15.4 & 1.1 & 14.9 & 1.0 \\
pgm-1 & 15.6 & 1.3 & 16.5 & 1.2 \\
\hline
\end{tabular}

\begin{tabular}{|c|cc|cc|}
\hline \multirow{3}{*}{ Plants } & AM & \multicolumn{2}{|c|}{ PM } \\
\cline { 2 - 5 } & $\begin{array}{c}{ }^{11} \text { C exuded from roots } \\
\text { (\%) }\end{array}$ & SE & $\begin{array}{c}{ }^{11} \text { C exuded from roots } \\
\text { (\%) }\end{array}$ & SE \\
\hline WT & 1.1 & 0.2 & 1.0 & 0.1 \\
sex1-1 & 1.6 & 0.2 & 0.8 & 0.1 \\
adg1-1 & 2.1 & 0.3 & 1.0 & 0.1 \\
pgm-1 & 1.0 & 0.1 & 1.1 & 0.1 \\
\hline
\end{tabular}




\section{A3. Chapter 4}

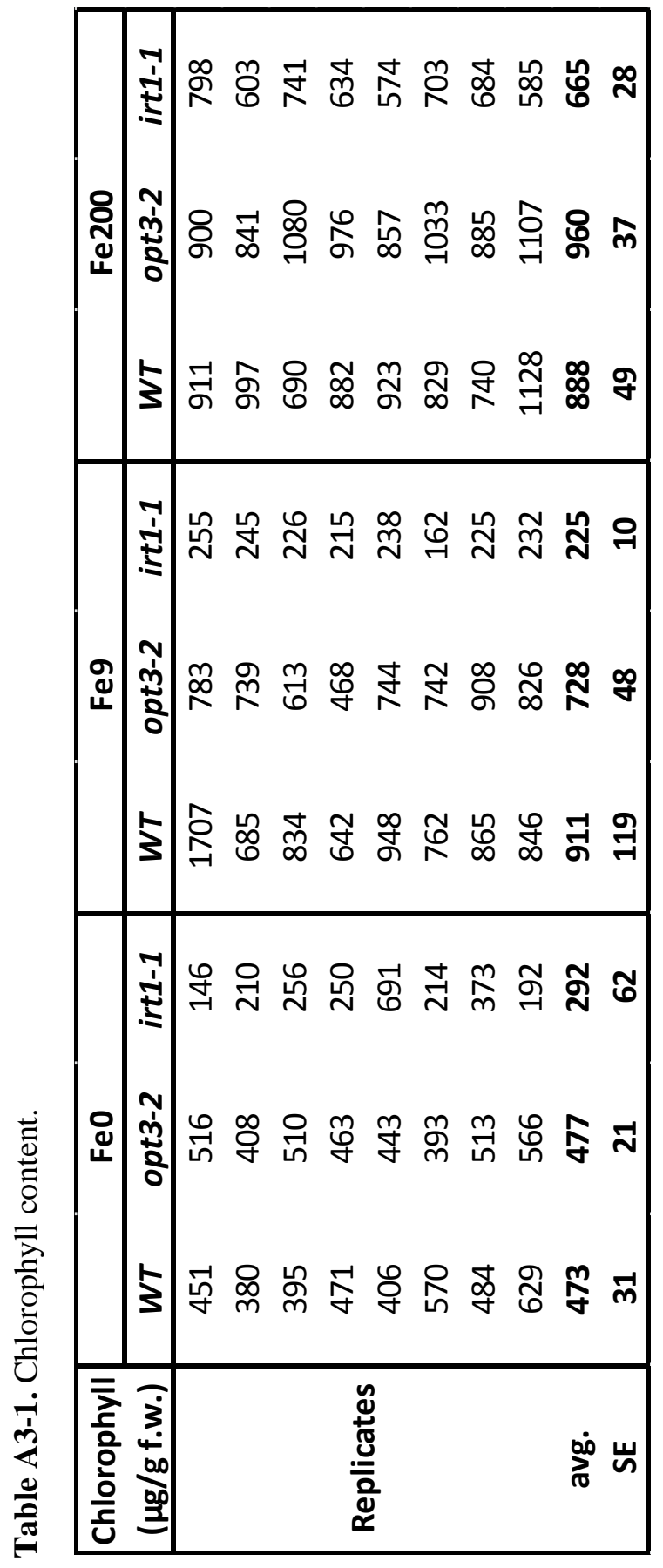




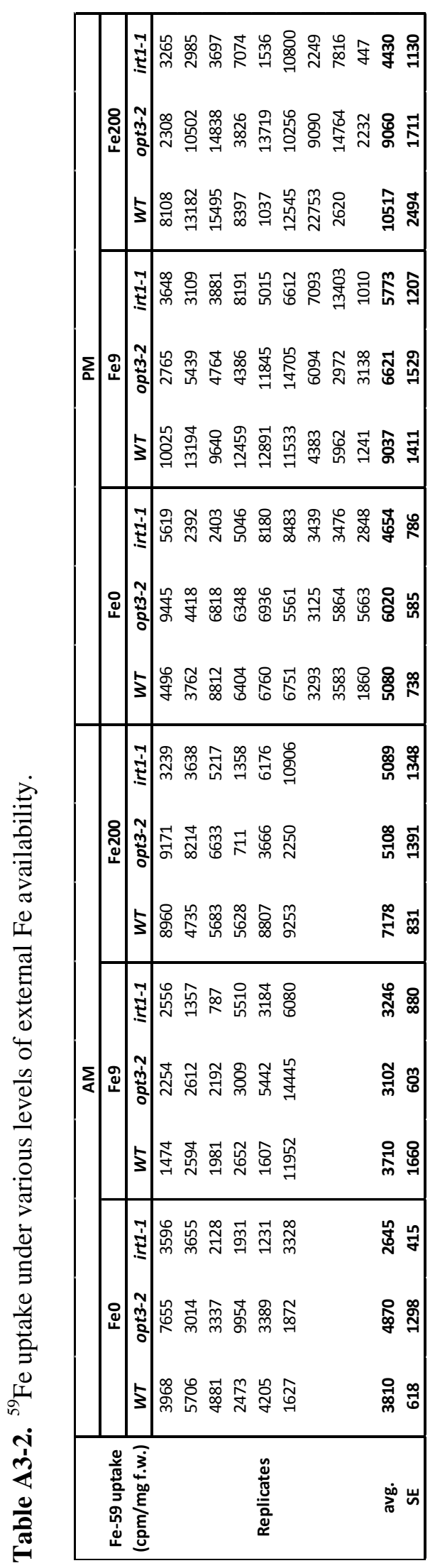




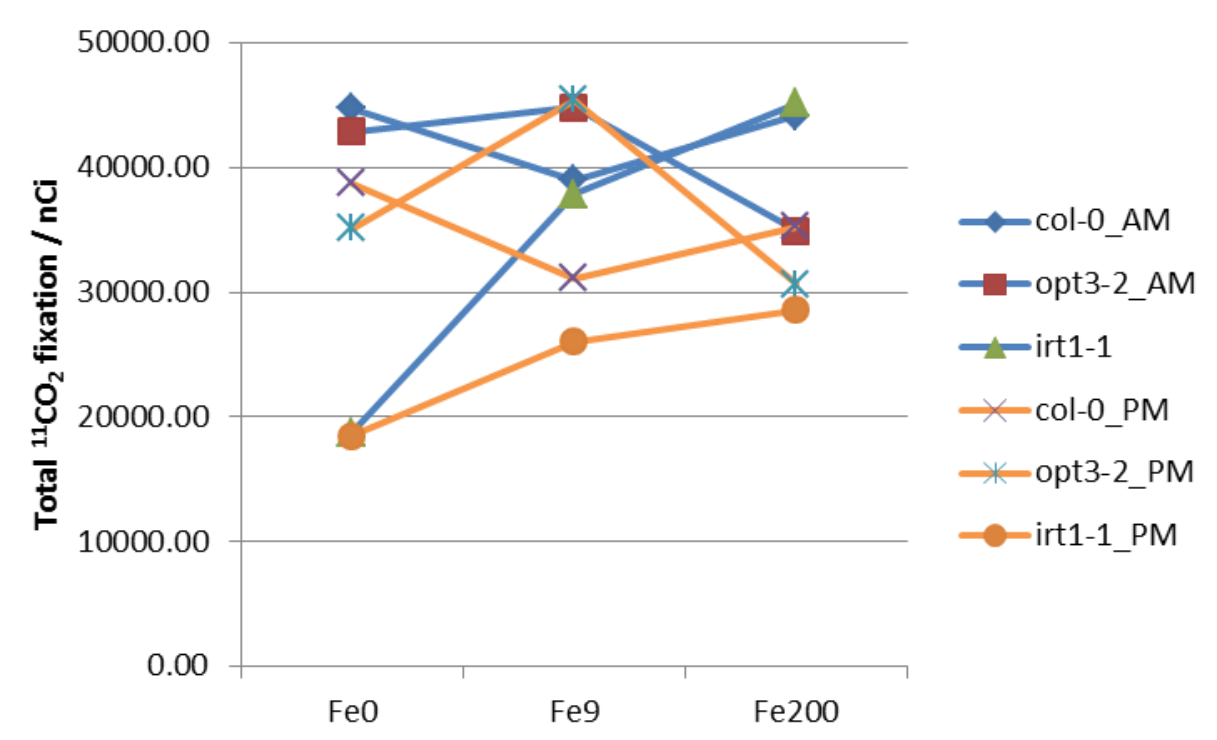

Figure A3-1. Total ${ }^{11} \mathrm{CO}_{2}$ fixation. The experiments were conducted both in the morning (A) and afternoon (B). $n=6-8$ seedlings. The statistics show the comparisons between the mutants and the WT under corresponding conditions. Standard errors were calculated, but not shown here. No statistically significant differences were found in this set of data. Error bars were omitted for clarity.

Table A3-3. Total ${ }^{11} \mathrm{CO}_{2}$ fixation.

\begin{tabular}{|c|c|cc|cc|}
\hline \multirow{2}{*}{$\begin{array}{c}\text { Fe } \\
\text { conditions }\end{array}$} & Plants & \multicolumn{2}{|c|}{ AM } & \multicolumn{2}{c|}{ PM } \\
\cline { 3 - 6 } & & $\begin{array}{c}\text { Total }{ }^{11} \text { C } \\
\text { fixation } \\
\text { (nCi) }\end{array}$ & SE & $\begin{array}{c}\text { Total }{ }^{11} \text { C } \\
\text { fixation (nCi) }\end{array}$ & SE \\
\hline \multirow{3}{*}{ Fe0 } & WT & 44698 & 2936 & 38701 & 6616 \\
& opt3-2 & 42813 & 2501 & 35074 & 3386 \\
& irt1-1 & 18747 & 2097 & 18462 & 2275 \\
\hline \multirow{3}{*}{ Fe9 } & WT & 38956 & 2287 & 31099 & 2285 \\
& opt3-2 & 44785 & 3445 & 45352 & 6403 \\
& irt1-1 & 37843 & 3504 & 26015 & 1686 \\
\hline \multirow{3}{*}{ Fe200 } & WT & 44144 & 4185 & 35266 & 3620 \\
& opt3-2 & 34924 & 3322 & 30660 & 2668 \\
& irt1-1 & 45094 & 6024 & 28585 & 2069 \\
\hline
\end{tabular}



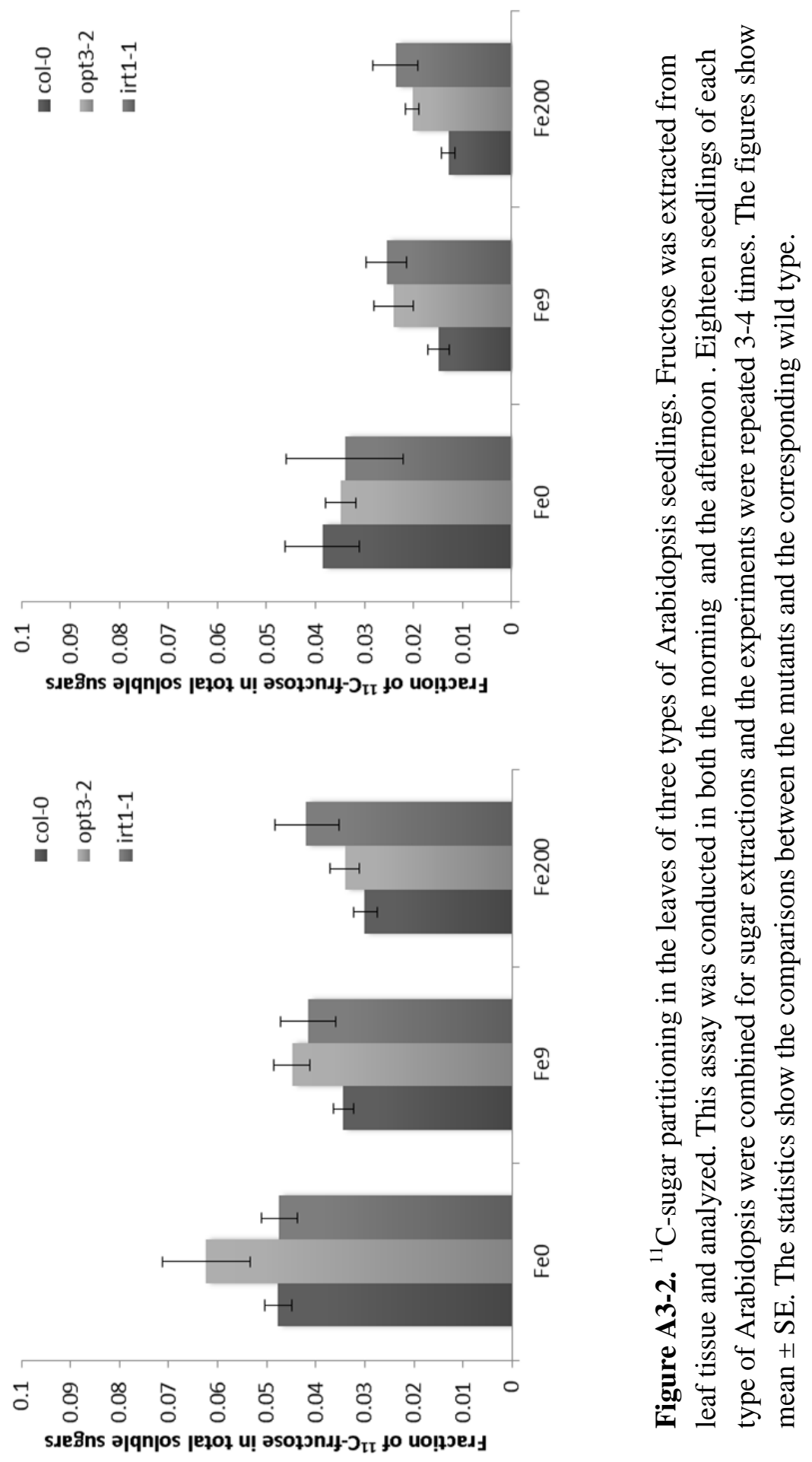

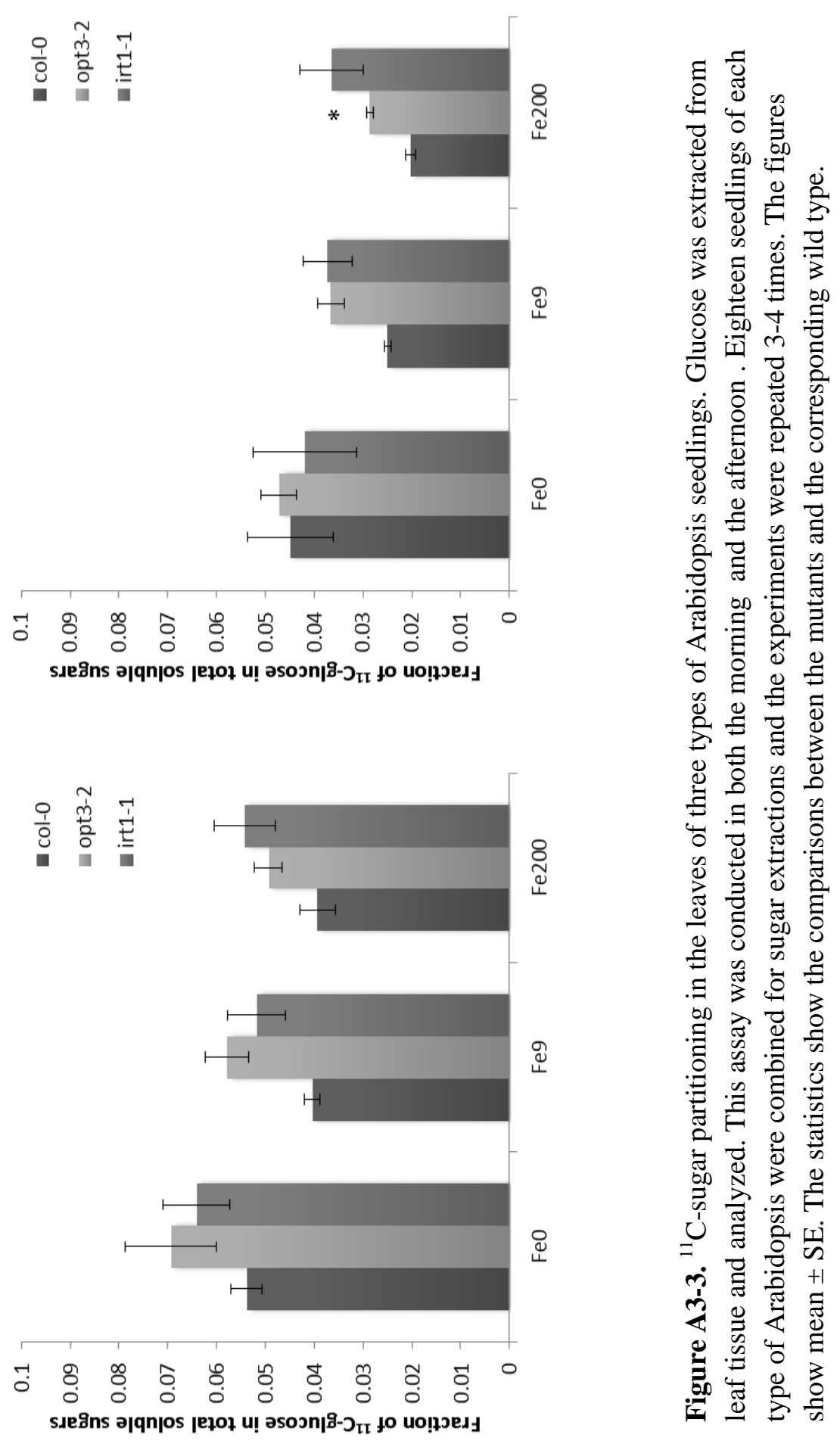

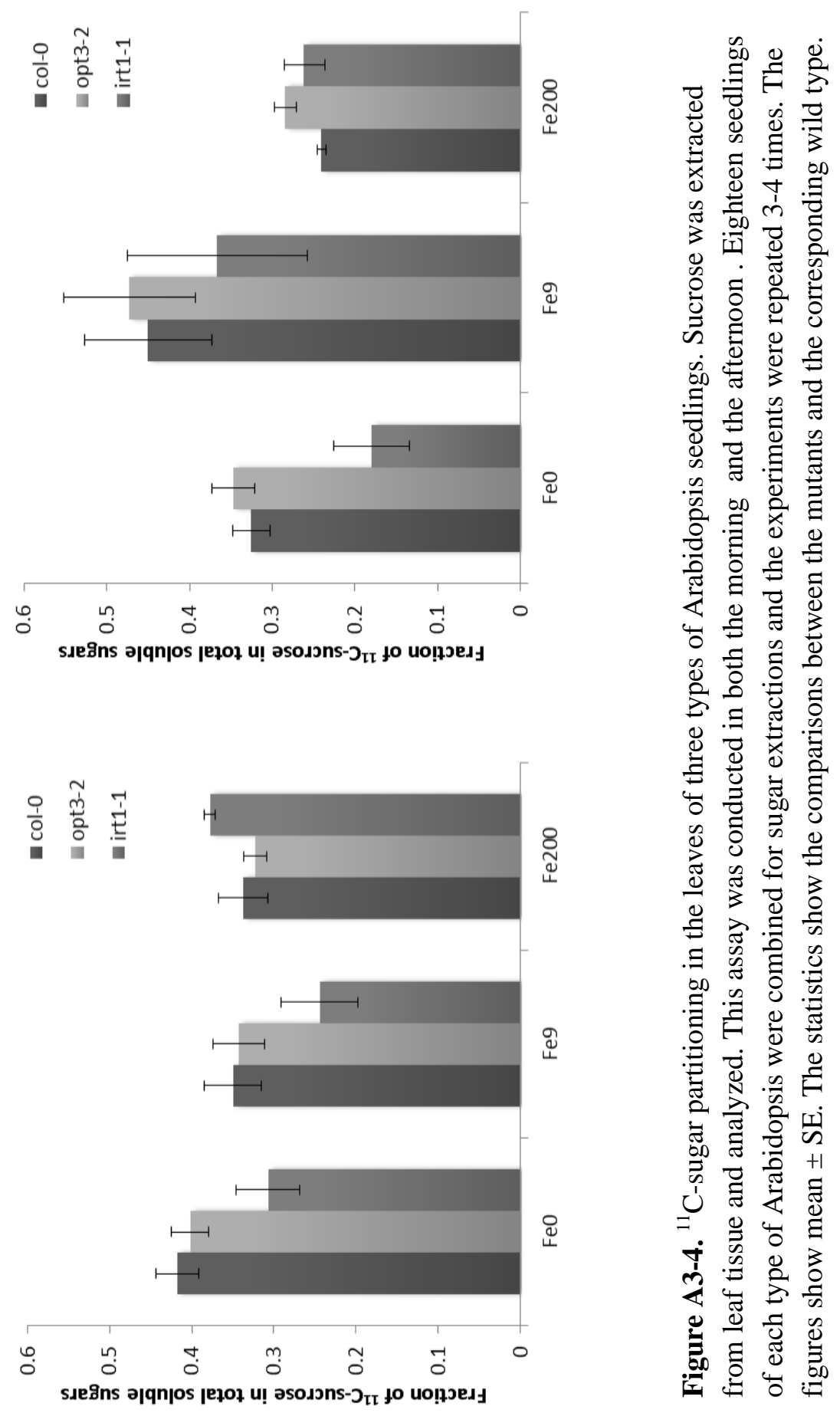
Table A3-4. ${ }^{11} \mathrm{C}$-sugar partitioning in the leaves of three types of Arabidopsis seedlings.

\begin{tabular}{|c|c|c|c|c|c|c|c|}
\hline \multirow[b]{3}{*}{ Time } & \multirow[b]{3}{*}{ Sugars } & \multicolumn{6}{|c|}{$\mathrm{FeO}$} \\
\hline & & $W T$ & & opt3-2 & & irt1-1 & \\
\hline & & $\begin{array}{l}\text { Percentage of } \\
\text { Soluble Sugars } \\
\qquad\left({ }^{11} \mathrm{C}\right)\end{array}$ & SE & $\begin{array}{l}\text { Percentage of } \\
\text { Soluble Sugars } \\
\qquad\left({ }^{11} \mathrm{C}\right)\end{array}$ & SE & $\begin{array}{l}\text { Percentage of } \\
\text { Soluble Sugars } \\
\qquad\left({ }^{11} \mathrm{C}\right)\end{array}$ & SE \\
\hline \multirow{4}{*}{ AM } & Fructose & 4.8 & 0.3 & 6.2 & 0.9 & 4.7 & 0.4 \\
\hline & Glucose & 5.4 & 0.3 & 6.9 & 0.9 & 6.4 & 0.7 \\
\hline & Sucrose & 41.7 & 2.6 & 40.2 & 2.3 & 30.7 & 3.9 \\
\hline & Fructose & 3.9 & 0.8 & 3.5 & 0.3 & 3.4 & 1.2 \\
\hline \multirow[t]{2}{*}{ PM } & Glucose & 4.5 & 0.9 & 4.7 & 0.4 & 4.2 & 1.1 \\
\hline & Sucrose & 32.5 & 2.3 & 34.7 & 2.5 & 18.0 & 4.6 \\
\hline \multirow[b]{3}{*}{ Time } & \multirow[b]{3}{*}{ Sugars } & \multicolumn{6}{|c|}{ Fe9 } \\
\hline & & \multicolumn{2}{|l|}{$W T$} & \multicolumn{2}{|l|}{ opt3-2 } & \multicolumn{2}{|l|}{ irt1-1 } \\
\hline & & $\begin{array}{l}\text { Percentage of } \\
\text { Soluble Sugars } \\
\qquad\left({ }^{11} \mathrm{C}\right)\end{array}$ & SE & $\begin{array}{c}\text { Percentage of } \\
\text { Soluble Sugars } \\
\qquad\left({ }^{11} \mathrm{C}\right)\end{array}$ & SE & $\begin{array}{l}\text { Percentage of } \\
\text { Soluble Sugars } \\
\qquad\left({ }^{11} \mathrm{C}\right)\end{array}$ & SE \\
\hline \multirow{4}{*}{ AM } & Fructose & 3.4 & 0.2 & 4.5 & 0.4 & 4.2 & 0.6 \\
\hline & Glucose & 4.0 & 0.2 & 5.8 & 0.4 & 5.2 & 0.6 \\
\hline & Sucrose & 35.0 & 3.5 & 34.3 & 3.2 & 24.5 & 4.7 \\
\hline & Fructose & 1.5 & 0.2 & 2.4 & 0.4 & 2.5 & 0.4 \\
\hline \multirow[t]{2}{*}{ PM } & Glucose & 2.5 & 0.1 & 3.7 & 0.3 & 3.7 & 0.5 \\
\hline & Sucrose & 45.0 & 7.7 & 47.3 & 8.0 & 36.7 & 10.9 \\
\hline \multirow[b]{3}{*}{ Time } & \multirow[b]{3}{*}{ Sugars } & \multicolumn{6}{|c|}{ Fe200 } \\
\hline & & $W T$ & & opt3-2 & & irt1-1 & \\
\hline & & $\begin{array}{l}\text { Percentage of } \\
\text { Soluble Sugars } \\
\qquad\left({ }^{11} \mathrm{C}\right)\end{array}$ & SE & $\begin{array}{l}\text { Percentage of } \\
\text { Soluble Sugars } \\
\qquad\left({ }^{11} \mathrm{C}\right)\end{array}$ & SE & $\begin{array}{l}\text { Percentage of } \\
\text { Soluble Sugars } \\
\qquad\left({ }^{11} \mathrm{C}\right)\end{array}$ & SE \\
\hline \multirow{4}{*}{ AM } & Fructose & 3.0 & 0.2 & 3.4 & 0.3 & 4.2 & 0.7 \\
\hline & Glucose & 3.9 & 0.4 & 4.9 & 0.3 & 5.4 & 0.6 \\
\hline & Sucrose & 33.7 & 3.0 & 32.3 & 1.4 & 37.8 & 0.7 \\
\hline & Fructose & 1.3 & 0.1 & 2.0 & 0.1 & 2.4 & 0.5 \\
\hline \multirow[t]{2}{*}{ PM } & Glucose & 2.0 & 0.1 & 2.9 & 0.1 & 3.7 & 0.7 \\
\hline & Sucrose & 24.0 & 0.5 & 28.4 & 1.3 & 26.1 & 2.5 \\
\hline
\end{tabular}


Table A3-5. ${ }^{11} \mathrm{C}$-carbohydrate relocation to belowground and root exudates.

\begin{tabular}{|c|c|c|c|c|c|}
\hline \multirow[b]{2}{*}{$\begin{array}{c}\text { Fe } \\
\text { conditions }\end{array}$} & \multirow[b]{2}{*}{ Plants } & \multicolumn{2}{|l|}{ AM } & \multicolumn{2}{|l|}{ PM } \\
\hline & & $\begin{array}{l}{ }^{11} \mathrm{C} \text { transported to } \\
\text { belowground (\%) }\end{array}$ & SE & $\begin{array}{l}{ }^{11} \mathrm{C} \text { transported to } \\
\text { belowground (\%) }\end{array}$ & SE \\
\hline \multirow{3}{*}{$\mathrm{FeO}$} & $W T$ & 4.0 & 0.3 & 1.9 & 0.4 \\
\hline & opt3-2 & 5.8 & 0.3 & 4.0 & 0.3 \\
\hline & irt1-1 & 8.2 & 1.4 & 4.5 & 0.7 \\
\hline \multirow{3}{*}{ Fe9 } & $W T$ & 5.7 & 0.5 & 2.3 & 0.2 \\
\hline & opt3-2 & 5.3 & 0.4 & 2.8 & 0.3 \\
\hline & irt1-1 & 7.0 & 0.4 & 4.6 & 0.6 \\
\hline \multirow{3}{*}{ Fe200 } & $W T$ & 6.2 & 1.1 & 1.2 & 0.1 \\
\hline & opt3-2 & 6.7 & 0.5 & 2.3 & 0.3 \\
\hline & irt1-1 & 6.6 & 1.3 & 2.1 & 0.2 \\
\hline
\end{tabular}

\begin{tabular}{|c|c|cc|cc|}
\hline \multirow{3}{*}{ Fe conditions } & \multirow{2}{*}{ Plants } & \multicolumn{2}{|c|}{ AM } & \multicolumn{2}{c|}{ PM } \\
\cline { 3 - 6 } & & $\begin{array}{c}{ }^{11} \text { C exuded to roots } \\
\text { (\%) }\end{array}$ & SE & $\begin{array}{c}{ }^{11} \text { C exuded to roots } \\
\text { (\%) }\end{array}$ & SE \\
\hline \multirow{3}{*}{ Fe0 } & WT & 2.5 & 0.4 & 0.6 & 0.2 \\
& opt3-2 & 3.3 & 0.3 & 0.8 & 0.2 \\
& irt1-1 & 6.0 & 1.2 & 1.1 & 0.3 \\
\hline \multirow{3}{*}{ Fe99 } & $\boldsymbol{W T}$ & 4.2 & 0.4 & 0.7 & 0.1 \\
& opt3-2 & 3.5 & 0.4 & 0.5 & 0.2 \\
& irt1-1 & 4.7 & 0.5 & 0.7 & 0.2 \\
\hline \multirow{3}{*}{ Fe200 } & WT & 4.6 & 1.1 & 0.5 & 0.1 \\
& opt3-2 & 5.6 & 0.7 & 0.7 & 0.3 \\
& irt1-1 & 4.7 & 1.3 & 0.8 & 0.2 \\
\hline
\end{tabular}




\section{References}

1. Zhang, Y., et al., Application of rhodamine B thiolactone to fluorescence imaging of $\mathrm{Hg}^{2+}$ in Arabidopsis thaliana. Sensors and Actuators B: Chemical, 2011. 153(1): p. 261-265.

2. Shi, W. and H. Ma, Rhodamine B thiolactone: a simple chemosensor for $\mathrm{Hg}^{2+}$ in aqueous media. Chem Commun (Camb), 2008(16): p. 1856-8. 
Appendix B: Protocols 


\section{B1. Small scale yeast transformation}

\section{Preparation for Competent Cells}

1. Inoculate $18 \mathrm{ml}$ of YPD (later $2 \mathrm{ml}$ of $20 \%$ sterile glucose is added) with three $3 \mathrm{~mm}$ colonies.

2. Shake media by hand to suspend cells.

3. Incubate at $30{ }^{\circ} \mathrm{C}$ for $16-18 \mathrm{hr}$ with shaking.

4. Transfer grown cells to $150 \mathrm{ml}$ of YPD.

5. Incubate at $30{ }^{\circ} \mathrm{C}$ for $3-4 \mathrm{hr}$.

6. Check OD value at $600 \mathrm{~nm}(0.5 \pm 0.05)$.

7. Place cells in a $250 \mathrm{ml}$ pre-chilled bottle and centrifuge at $4500 \mathrm{rpm}$ for $5 \mathrm{~min}$ at $4{ }^{\circ} \mathrm{C}$.

8. Discard the supernatant and suspend cell pellets in $50 \mathrm{ml}$ of sterile $\mathrm{ddH}_{2} \mathrm{O}$ and shake by hand.

9. Centrifuge at $4500 \mathrm{rpm}$ for $5 \mathrm{~min}$ at $4{ }^{\circ} \mathrm{C}$.

10. Decant the supernatant.

11. Resuspend the cell pellet in $50 \mathrm{ml}$ of freshly prepared, sterile $1 \mathrm{X}$ TE/LiAc.

12. Centrifuge at $4500 \mathrm{rpm}$ for $5 \mathrm{~min}$ at $4{ }^{\circ} \mathrm{C}$.

13. Resuspend the cell pellet in 3-5 $\mathrm{ml}$ of $1 \mathrm{X} \mathrm{TE} / \mathrm{LiAc}$.

Note: Keep $4{ }^{\circ} \mathrm{C}$ from step 7 to 13 . 


\section{Plasmid Transformation}

1. Prepare PEG/LiAc solution and plasmids needed to be transformed.

2. Add $100 \mu \mathrm{l}$ of yeast competent cells into $1.5 \mathrm{ml}$ Eppendorf tubes with $\sim 500 \mathrm{ng}$ of plasmids. (sometimes DNA carrier is needed)

3. Add $600 \mu \mathrm{l}$ of $40 \% \mathrm{PEG} / 1 \mathrm{X}$ TE/LiAc solution and vortex at high speed to mix.

4. Incubate at $30{ }^{\circ} \mathrm{C}$ for 30 min with shaking.

5. Heat shock for $15 \mathrm{~min}$ in a $42{ }^{\circ} \mathrm{C}$ water bath.

6. Chill cells on ice for $5 \mathrm{~min}$.

7. Centrifuge cells for $5 \mathrm{sec}$ at room temperature.

8. Remove the supernatant.

9. Quick spin down cells and remove the supernatant.

10. Resuspend cells in $500 \mu \mathrm{l}$ of sterile $\mathrm{ddH}_{2} \mathrm{O}$.

11. Spread $100 \mu \mathrm{l}$ of cells on SD agar medium.

12. Incubate at $30^{\circ} \mathrm{C}$ for $2-3$ days.

YPD: Yeast Extract Peptone Dextrose (commercially available)

SD Medium: Synthetic Defined medium

\section{$\underline{1 X \text { TE/LiAc }}$}

10 mM Tris- $\mathrm{HCl}$ pH 8.0

$1 \mathrm{mM}$ EDTA

0.1 M Lithium acetate 


\section{PEG/LiAc}

40\% PEG-4000

0.1 M Lithium actetate

\section{PEG/ 1X TE/LiAc}

40\% PEG-4000

10 M Tris-HCl pH 8.0

1 mM EDTA

$0.1 \mathrm{M}$ Lithium actetate 


\section{B2. Plasmid DNA Extraction from Yeast for Use in the Transformation}

\section{of $E$. coli}

1. Inoculate yeast in $3 \mathrm{ml} \mathrm{SD}-U r a$ media and incubate at $30{ }^{\circ} \mathrm{C}$ with shaking overnight.

2. Centrifuge $1 \mathrm{ml}$ of culture at $14,000 \mathrm{x} \mathrm{g}$ for $30 \mathrm{sec}$ at room temperature and remove all residual liquid.

3. Rinse the cell pellet by $1 x$ TE buffer.

4. Suspend the cells in $100 \mu$ freshly prepared $3 \%$ sodium dodecyl sulfate (SDS).

5. Incubate $15 \mathrm{~min}$ at room temperature with occasional mixing by several rapid inversions.

6. Add $500 \mu \mathrm{TE}$ buffer. Mix completely by several rapid inversions.

7. Add $60 \mu 13 \mathrm{M}$ sodium acetate. Mix completely by several rapid inversions.

8. Add $600 \mu \mathrm{l}$ phenol:chloroform:isoamyl alcohol, 25:24:1.

9. Vortex for $2 \mathrm{~min}$ at full speed.

10. Centrifuge in a microcentrifuge at $14,000 \mathrm{x} g$ for $2 \mathrm{~min}$. Transfer the upper aqueous phase to a new microcentrifuge tube.

11. Repeat steps 8-10.

12. Add $1 \mathrm{ml}$ of $100 \%$ ethanol. Mix completely by several rapid inversions.

13. Incubate at $-80{ }^{\circ} \mathrm{C}$ for at least $20 \mathrm{~min}$.

14. Centrifuge in a microcentrifuge at $14,000 \mathrm{x} \mathrm{g}$ for $20 \mathrm{~min}$.

15. Add $100 \mu \mathrm{l}$ of $70 \%$ ethanol and incubate for $1 \mathrm{hr}$.

16. Centrifuge in a microcentrifuge at $14,000 \mathrm{x} \mathrm{g}$ for $5 \mathrm{~min}$.

17. Discard the supernatant. Centrifuge for $10 \mathrm{sec}$ and remove all residual supernatant. 18. Suspend the pellet in $50 \mu \mathrm{l} \mathrm{H}_{2} \mathrm{O}$. 
TE buffer

10 mM Tris- $\mathrm{HCl}$ pH 8.0

$1 \mathrm{mM}$ EDTA 


\section{B3. Modified Hoagland's solution (without Fe)}

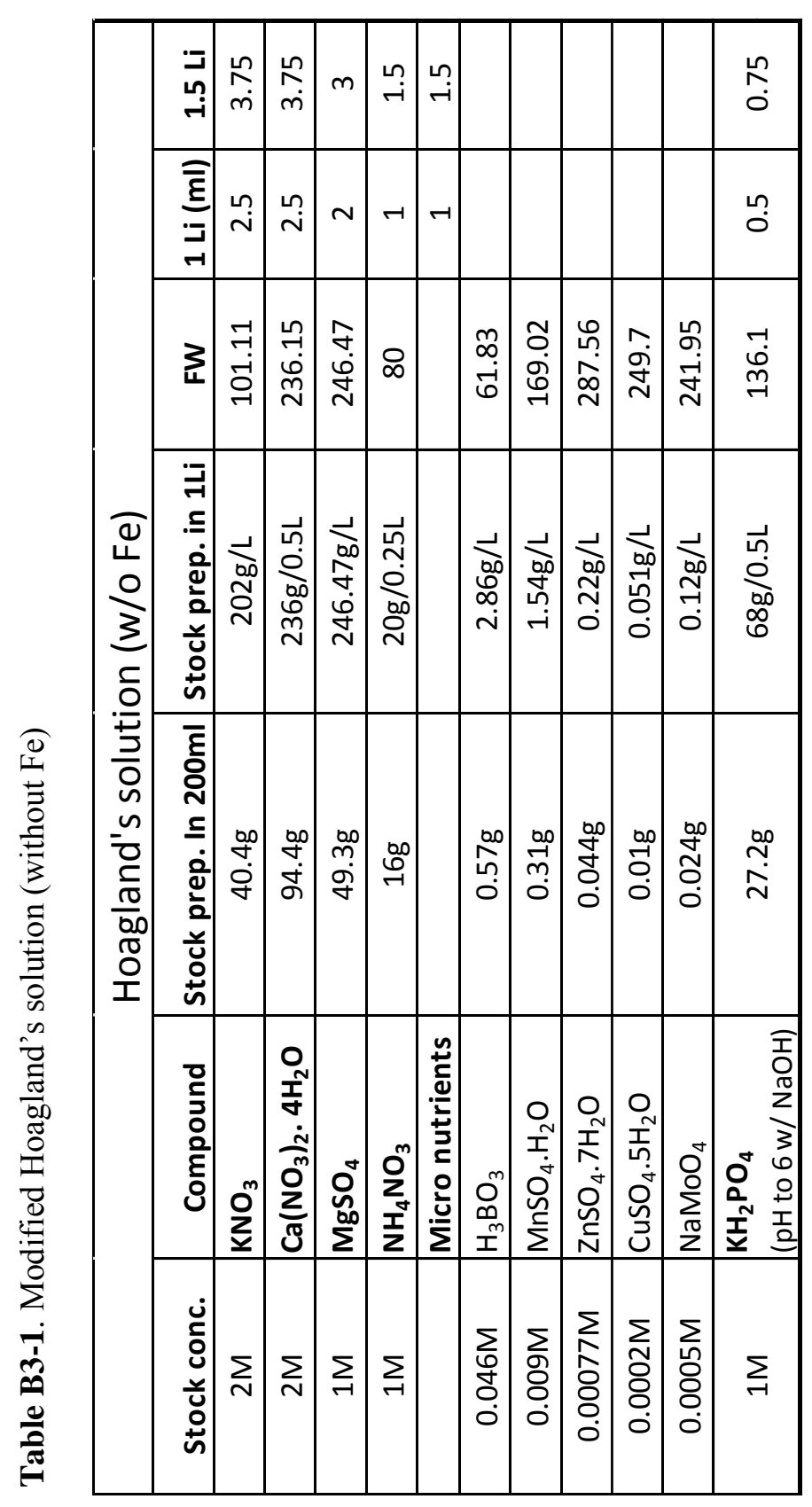




\section{VITA}

Lihui Song was born and raised in Tianjin, China. She received her bachelor's degree in Applied Chemistry from the Tianjin University (Tianjin, China) in May 2008. To extend her knowledge and experience, Lihui chose to pursuit a further education in US. She was accepted as a Ph.D. student in the Chemistry Department at University of Missouri where she joined Jurisson research group and earned her Ph.D. degree in 2013. In the same year, she received the Mizzou Advantage Prepare for Future Faculty fellowship from University of Missouri, specifically in the One Health/One Medicine area. During the fellowship, she will work with Dr. Scott Frey in the MU Brain Imaging Center (BIC) on Magnetic Resonance Spectroscopy (MRS) studies on human brains, as well as teach several courses related to her research. 